University of Denver

Digital Commons @ DU

8-1-2011

\title{
Posttraumatic Growth Following Cancer: The Role of Cognitive Processing, Anxiety, Depression and Perceived Threat
}

Jennifer M. Caspari

University of Denver

Follow this and additional works at: https://digitalcommons.du.edu/etd

Part of the Counseling Psychology Commons

\section{Recommended Citation}

Caspari, Jennifer M., "Posttraumatic Growth Following Cancer: The Role of Cognitive Processing, Anxiety, Depression and Perceived Threat" (2011). Electronic Theses and Dissertations. 116.

https://digitalcommons.du.edu/etd/116

This Dissertation is brought to you for free and open access by the Graduate Studies at Digital Commons @ DU. It has been accepted for inclusion in Electronic Theses and Dissertations by an authorized administrator of Digital Commons@DU. For more information, please contact jennifer.cox@du.edu,dig-commons@du.edu. 
POSTTRAUMATIC GROWTH FOLLOWING CANCER: THE ROLE OF

COGNITIVE PROCESSING, ANXIETY, DEPRESSION AND PERCEIVED THREAT

\author{
A Dissertation \\ Presented to \\ the Faculty of the Morgridge College of Education \\ University of Denver \\ In Partial Fulfillment \\ of the Requirements for the Degree \\ Doctor of Philosophy
}

by

Jennifer M. Caspari, M.A.

August 2011

Advisor: Cynthia McRae, Ph.D. 
Author: Jennifer M. Caspari, M.A.

Title: Posttraumatic Growth Following Cancer: The Role of Cognitive Processing, Anxiety, Depression and Perceived Threat

Advisor: Cynthia McRae, Ph.D.

Degree Date: August 2011

\section{Abstract}

This study explored several predictors of posttraumatic growth (PTG) in a sample of 169 breast, prostate and colorectal cancer survivors. The first aim was to determine the influence of Anxiety, Depression and Perceived Threat (defined as the combination of Life Outlook Threat, i.e., the degree a cancer diagnosis challenged a survivor's assumptive world, and Physical Threat, i.e., threat to mortality and physical well-being) in the prediction of Positive and Negative Cognitive Processing. The second aim was to examine the effect of Anxiety, Depression, Perceived Threat, and Positive and Negative Cognitive Processing in the predication of PTG.

Cancer survivors who were treated at one of the Denver Division clinics of the Rocky Mountain Cancer Center participated in the study. Since little empirical research has been conducted utilizing the variable of cognitive processing in the psychological literature, the study sought to investigate how Anxiety, Depression, and Perceived Threat were related to Positive and Negative Cognitive Processing. Hierarchical regression analyses were used to explore four primary hypotheses.

The results of the study revealed several important findings. Physical Threat, Depression, and Permanent After-Effects of Cancer Treatment significantly predicted Positive Cognitive Processing, with lower levels of Physical Threat and Depression and no After-Effects of Treatment predicting higher Positive Cognitive Processing. Life Outlook Threat, Positive Cognitive Processing, and Type of Cancer Treatment Received 
also significantly predicted PTG. The findings indicated that greater life outlook threat and positive cognitive processing as well as receiving more than one form of cancer treatment predicted greater growth. None of the variables reached significance in predicting Negative Cognitive Processing and Negative Cognitive Processing failed to significantly predict PTG.

While perceived threat and cognitive processing have a strong theoretical basis in the emergence of growth, the constructs have received little empirical attention. This is the first study that has assessed how being diagnosed with cancer challenges, rather than alters, a survivor's assumptive world. The results of the study provide evidence that increases in life outlook threat and positive cognitive processing are related to PTG. 


\section{Acknowledgments}

I could not have completed this dissertation without the support, encouragement and guidance of many people, and it is with much gratitude that I express my appreciation to these individuals. To Dr. Cynthia McRae for her unwavering encouragement, kindness, assistance and valuable feedback throughout the entire dissertation process. To Dr. Ruth Chao for her upbeat demeanor, optimism and helpful comments. Dr. Carly Parry has been an inspiration to me throughout this process and her thought provoking feedback and beneficial guidance helped to shape this dissertation into a worthwhile endeavor. In addition, I want to thank Dr. Roger Salters for his generosity and eagerness in chairing the defense meeting. I also want to acknowledge the cancer survivors who embraced this research project and took the time to provide me with valuable information regarding their cancer experiences.

To my parents and family for their unconditional love and support and always believing that I can accomplish anything I set my mind to. To my Dad for reading numerous drafts of this dissertation and providing useful comments, and to my Mom for helping to keep things in perspective on challenging days. Finally, with the deepest thanks I want to recognize my cherished friends who provided support whenever needed. I want to especially acknowledge my good friend Sarah Lawless who went through her own challenging life circumstance with bravery and zest as I wrote this dissertation. I could not have reached my academic goals without the support and love of those around me - Thank You! 
Table of Contents

Chapter One: Introduction.............................................

Background of the Problem........................................

Statement of the Problem...............................................5

Purpose of Studying the Problem.................................... 7

Importance of Studying the Problem..................................9

Overview of Hypotheses.........................................11

Overview of Variables and Measures................................12

Limitations..................................................... 13

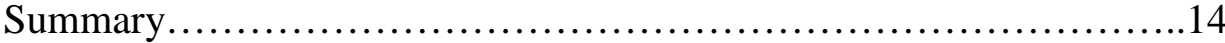

Chapter Two: Review of Selected Literature............................16

Introduction..................................................16

Why Cancer as a Model of PTG..................................18

Trauma..........................................................20

Trauma and Assumptive Beliefs..................................25

Posttraumatic Growth............................................28

Overview.....................................................28

Model of Posttraumatic Growth.........................................30

Challenges to the Concept of PTG..................................33

Cognitive Processing and Posttraumatic Growth............................36

Unique Aspects of the Cancer Experience............................41

Experience of Being a Survivor: Role of Fear \& Anxiety...............42

Cancer and Posttraumatic Growth...................................45

Key Variables Associated with PTG Following Cancer..................47

Demographic Variables............................................47

Age............................................................ 48

Gender.....................................................49

Time............................................................53

Types of Cognitive Processing .......................................56

Perceived Threat: Overview.......................................58

Curvilinear Relationship Between Threat and Growth......................58

Threat to Physical Well-Being........................................60

Threat to Assumptive World.......................................66

Anxiety \& Depression in Cancer Patients..............................69

Differences b/t Cognitions Assoc w/Anxiety \& Depression..................73

The Role of Cognition in Anxiety \& Depression.......................75

Types of Cancer.........................................................

Summary .................................................. 81

Chapter Three: Methods...........................................83

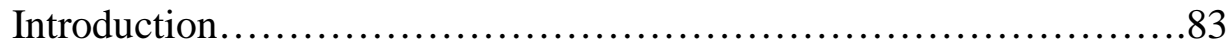

Participants................................................... 83

Power and Sample Size............................................84 


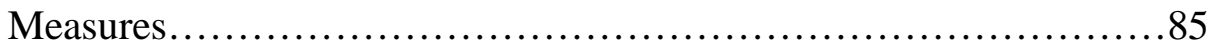

Demographics................................................... 85

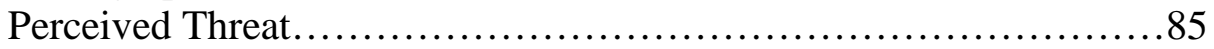

Anxiety and Depression......................................... 86

Cognitive Processing .................................................... 89

Posttraumatic Growth...........................................91

Procedure ....................................................... 93

Data Analyses.................................................

Preliminary Analyses.............................................96

Primary Analyses................................................ 97

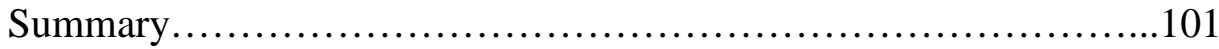

Chapter Four: Results................................................103

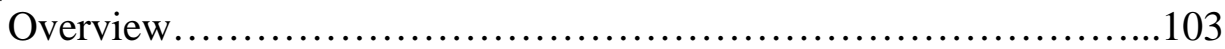

Preliminary Analyses............................................... 103

Survey Details and Response Rate.................................104

Analysis of Missing Data...........................................105

Demographic Information........................................

Reliability Analyses Related to Main Variables........................110

Descriptive Statistics for Independent and Dependent Variables.......112

Mean Comparisons for Variables Between Two Groups...............114

Mean Comparisons for Variables Between Three or More Groups.....116

Primary Analyses.................................................119

Statistical Analyses Addressing Research Hypotheses..................122

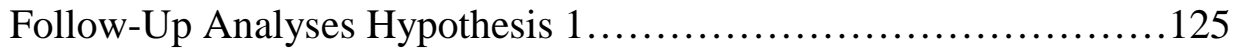

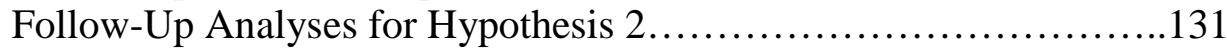

Follow-Up Analyses for Hypothesis 3 ..............................134

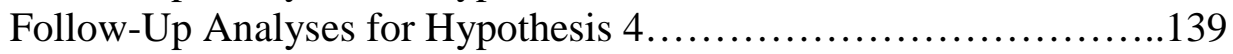

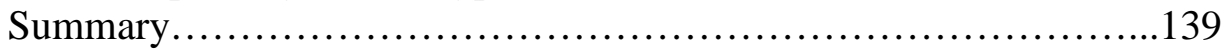

Chapter Five: Discussion.............................................. 142

Summary of the Study .........................................142

Specific Findings and Implications for Hypotheses....................144

Summary of Study Implications................................153

Study Limitations............................................. 156

Recommendations for Future Research............................159

Conclusions........................................................ 162

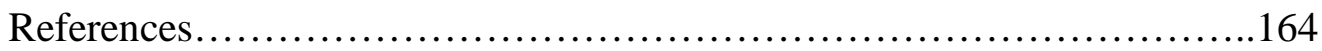

Appendices

Appendix A: Glossary of Terms...................................184

Appendix B: Informed Consent..................................187

Appendix C: HIPAA Research Authorization.........................191

Appendix D: Demographics.........................................194

Appendix E: Perceived Threat Questionnaire.......................198 
Appendix F: Hospital Anxiety and Depression Scale................200

Appendix G: Cognitive Processing of Trauma Scale.................203

Appendix H: The Posttraumatic Growth Inventory..................205 
List of Tables

Table 1. Percentage of Individuals Diagnosed with Breast, Prostate and Colorectal Cancer Based on Age................................79

Table 2. Percentage of Individuals Diagnosed with Stage I-IV Cancer........80

Table 3. Overview of Demographic Characteristics.......................... 106

Table 4. Overview of Cancer Diagnosis and Treatment Information.........108

Table 5. Descriptive Statistics for Independent and Dependent Variables...113

Table 6. Correlation Coefficients....................................113

Table 7. Hierarchical Regression of Age, Gender, Stage of Diagnosis, Time Since Treatment Completion, Recurrence, and Interference of After-

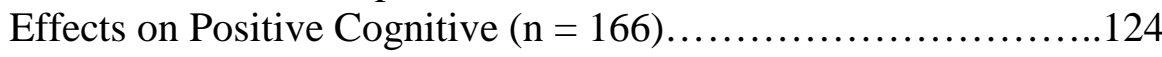

Table 8. Hierarchical Regression of Age, Gender, Stage of Diagnosis, Time Since Treatment Completion, Cancer Type Treatment Received, Life Outlook Threat, Physical Threat, Anxiety, Depression, and Positive Cognitive Processing on Posttraumatic Growth $(n=165) \ldots \ldots \ldots . . .128$

Table 9. Hierarchical Regression of Age, Gender, Stage of Diagnosis, Time Since Treatment Completion, Cancer Type, Location of Treatment and Interference of After-Effects on Negative Cognitive Processing

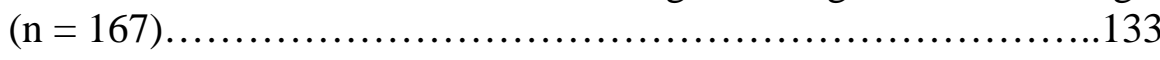

Table 10. Hierarchical Regression of Age, Gender, Stage of Diagnosis, Time Since Treatment Completion, Cancer Type, Treatment Received, Life Outlook Threat, Physical Threat, Anxiety, Depression, and Negative Cognitive Processing on Posttraumatic Growth $(\mathrm{n}=$ 165).... 


\section{List of Figures}

Figure 1. A model of posttraumatic growth............................. 32 


\section{CHAPTER ONE}

\section{STUDY OVERVIEW}

Introduction

\section{Background of the Problem}

Cancer is a potentially terminal illness that impacts many people. In 2008, nearly one and a half million men and women were predicted to be diagnosed with cancer, and over a half million individuals died of cancer in the United States (National Cancer Institute, 2009). Receiving a cancer diagnosis is typically a frightening and unexpected event that challenges an individual's fundamental beliefs about him/herself and the predictability of the world. Cancer is known to be a stressful and often traumatic experience affecting many aspects of a person's life. Tedeschi and Calhoun (1995) described characteristics of a traumatic event as being sudden, unexpected, uncontrollable, and producing ongoing effects. Cancer leads to confrontation of one's mortality, and the inevitability of death (Tallman, Altmaier, \& Garcia, 2007). Although medical advances have been made, cancer remains a life-threatening illness, often provoking fear and uncertainty about the future. Survivors typically deal with numerous negative experiences, including medical treatments and their side effects, such as pain, 
fatigue, nausea, and hair loss; temporary and permanent changes in physical appearance; alterations in future life plans; the threat of future disease recurrence, and changes in social roles and relationships (Manne, Ostroff, Winkel, Goldstein, Fox \& Grana, 2004; Stanton, Bower, \& Low, 2006).

Research in the psycho-oncology field has historically focused on the negative psychological consequences of cancer. Individuals with cancer often exhibit symptoms of psychological distress, including depression, anxiety, and manifestations of posttraumatic stress disorder (Tallman et al., 2007). Yet, researchers suggest that severe affective disturbance is relatively rare, and on the whole, not enduring, as most survivors resume normal mood and functioning within the year after medical treatment completion (Andersen, Anderson, \& deProsse, 1989). Thus, research focused only on documenting distress and dysfunction may lead to a potentially misleading conclusion about adjustment following cancer.

The notion that suffering and distress can be potential sources of positive change dates back to writings by the ancient Hebrews, Greeks, early Christians, and Buddhists, all of whom noted the potentially transformative power of suffering (Tedeschi \& Calhoun, 1995). The past decade has seen increased empirical focus on the potential for positive change and growth in the aftermath of trauma. Cancer is one area where growth is possible. A growing body of research supports the idea that cancer diagnosis and treatment might result in positive psychological outcomes as some cancer survivors report profound positive changes in themselves, their relationships with others, and other life areas following cancer. 
Many terms have been applied to the idea of positive change after trauma, including benefit finding, stress-related growth, found meaning, adversarial growth, perceived benefits, thriving, and posttraumatic growth, each with slightly different definitions. The lack of uniformity of terminology has caused confusion and a fragmented understanding of the phenomenon of posttraumatic growth. This study focused on the concept of posttraumatic growth (PTG), defined by Tedeschi and Calhoun (2004) as "positive psychological change experienced as a result of the struggle with highly challenging life circumstances" (p. 1).

PTG encompasses the experiences of individuals whose development, at least in some areas, has surpassed what was present before the struggle with a crisis. The individual has not only survived, but has experienced changes that are perceived as significant, and go beyond a return to normal functioning. PTG is not a return to baseline; rather, it is an experience of positive change that, for some, is profound. Unlike similar concepts of resilience, sense of coherence, or hardiness, PTG has a quality of transformation. It involves a movement beyond pre-trauma levels of functioning (Tedeschi \& Calhoun, 2004).

Tedeschi and Calhoun (2004) proposed that growth is not a direct result of a trauma, but rather an individual's struggle with the new reality in the aftermath of the trauma, which is critical in determining the extent to which PTG occurs. Researchers have suggested that people develop and rely on a general set of assumptions and beliefs about the world that guide their actions, and lend understanding and meaning to events. Major life crises challenge a person's understanding of the world, and psychological crisis can be defined as the degree to which the event challenges a person's assumptive 
world, including assumptions about benevolence, predictability, controllability of the world, one's safety, and one's identity and future (Janoff-Bulman, 1992).

The theory of PTG suggests that the traumatic event that occurs must be challenging enough to the assumptive world of the individual in order to be a catalyst for the cognitive processing necessary for growth. In other words, there must be a sufficient amount of perceived threat to one's beliefs and well-being for PTG to develop. Thus, a prerequisite for PTG is threat to one's life outlook (Tedeschi \& Calhoun, 2004). In the current study, Life Outlook Threat is defined as the degree to which being diagnosed with cancer challenged the survivor's assumptive beliefs and worldview. If a person's preexisting beliefs are not disrupted by the event, there is no need for adjustment and reevaluation, and therefore, no opportunity for change. Once an individual's worldview is altered, or perhaps even shattered by a traumatic event, cognitive processing and restructuring are among the processes necessary to "rebuild" one's beliefs (JanoffBulman, 1992).

Cognitive processing is defined by Bower, Kemeny, Taylor, and Fahey (1998, p. 979) as "the process of actively thinking about a stressor, the thoughts and feelings it evokes, and its implications for one's life and future." Williams-Avery (1999) created the Cognitive Processing of Trauma Scale, which describes cognitive processing using five factors: Positive Cognitive Restructuring, Resolution, Downward Comparison, Denial, and Regret. It is proposed that Positive Cognitive Restructuring, Resolution, and Downward Comparison represent Positive Cognitive Processing, while Denial and Regret indicate Negative Cognitive Processing. Williams-Avery (1999) explained that improved cognitive processing may signify "reduced signs of repression, such as avoidant/numbing 
behavior and intrusive thoughts, greater organization of thoughts, higher degrees of assimilation or meaning-making, greater ability to see the experience from alternative and often more positive perspectives, and greater acceptance and resolution" (p. 119).

This study focused on how cognitive processing influences PTG. Tedeschi and Calhoun (2004) asserted that the degree to which the person is engaged cognitively by the trauma is a central element in the process of PTG. It is suggested that people who report growth in the aftermath of trauma must alter certain goals and basic assumptions that they held prior to the trauma, while at the same time persisting in an attempt to build new beliefs, goals, and meanings. Tedeschi and Calhoun (2004) argued that persistence in cognitive processing should be associated with PTG.

Calhoun and Tedeschi (2006) differentiated between different types of cognitive processing that occur at different points in time as individuals cope with trauma. They explained that immediately following a crisis an individual engages in a ruminative process that is typically automatic and intrusive. As the person works to manage emotional distress, processing becomes more deliberate and effortful, which is the type of cognitive processing believed to produce PTG. PTG is more likely when a person ruminates in a deliberate, reflective way, trying to make sense out of the trauma. Deliberate and reflective rumination tends to assist in repairing, restructuring, and rebuilding the individual's general way of understanding the world, and is proposed to be qualitatively different from the nonproductive and often intrusive rumination that is characteristic of depression and anxiety. 


\section{Statement of the Problem}

Based on published rates between 2004-2006, 40.58\% of men and women born today will be diagnosed with cancer of all types sometime during their lifetime. This number can also be expressed as almost one in two men and women will be diagnosed with cancer of all types sometime during their lifetime (National Cancer Institute, 2009). It is commonly thought that a cancer diagnosis and subsequent treatment are often associated with negative psychological responses, at least initially. However, a growing literature also testifies to the prevalence of PTG resulting from the struggle with cancer. It is important to recognize and acknowledge that positive life changes and personal growth can occur following cancer, and that changes suggest the possibility of the transformative power of illness and human resilience, which can be enhanced through psychological intervention. At present, researchers lack a comprehensive understanding of the process of PTG, specifically in regard to cognitive processing. It is possible that research on PTG may yield a more complete and balanced understanding of cancer survivors' psychosocial experience and health. Cognitive engagement following trauma is a key factor in Tedeschi and Calhoun's (2004) model of PTG; however, few studies have examined how cognitive factors are connected to growth. Specifically, how are Positive and Negative Cognitive Processing reflected in the Cognitive Processing of Trauma Scale each related to PTG? A better understanding of the types of cognitive processing, following a cancer diagnosis, that lead to growth might direct psychological interventions targeting cognitions that have the potential to facilitate PTG.

When studying cognitive processing, it is imperative to consider factors that influence a person's cognitions and ability to process information. Individuals with 
cancer often exhibit symptoms of depression and anxiety (Roy-Byrne, Davidson, Kessler, Asmundson, Goodwin, Kubzansky, et al., 2008; Smith, Gomm, Dickens, 2003), which in many instances are prompted by the degree of perceived threat that persons experience. Research has indicated that these symptoms influence how an individual thinks and the content and valence of thoughts (Watkins, 2008). Typically, both depression and anxiety increase negative affect, which often hinders effective cognitive processing. Mineka and Sutton (1992) reported that anxiety and depression have substantial effects on the processing of emotionally relevant information. For instance, anxiety seems to be related to attentional bias for threatening stimuli, and depression appears to be associated with memory bias for negative self-referential material (Mineka \& Sutton, 1992).

Depressed individuals tend to engage in more negative and self-critical rumination that can be maladaptive; they may also experience impaired concentration and problem solving. Furthermore, anxiety symptoms often lead to an overestimation of threat, underestimation of coping resources, and overuse of compensatory self-protective strategies, such as cognitive and behavioral avoidance (Riskind, Williams \& Joiner, 2006). As stated previously, PTG requires deliberate and effortful cognitive processing, and since anxiety and depression often lead to rigid and unconstructive ruminative thinking, high levels of anxiety and depression will likely impede PTG.

Thus, it would be remiss to examine ways in which cognitive processing is related to PTG without also examining how a cancer survivor's level of perceived threat, anxiety and depression influence cognitive processing. For instance, if it is the case that higher levels of anxiety and depression hinder positive cognitive processing, anxious and depressive symptoms may be decreased through psychological intervention, creating a 
greater capacity for a cancer survivor to engage in cognitive processing that might aid growth.

\section{Purpose of Studying the Problem}

Research supports the notion that many individuals perceive that they have grown or benefited in some way from their experiences with cancer (Stanton, Bower, \& Low, 2006). While PTG is transformative in nature and represents a change beyond recognition of benefit, its emphasis on positive change and the transformative power of trauma, has similarities with the framework of positive psychology, which focuses on positive features of the human experience, such as happiness, hope, and wisdom (Seligman \& Csikszentmihalyi, 2000). The traditional pathology model of psychology neglects the strengths of individuals, focuses solely on problems (which yields a limited view of the person) and does not work to build adaptive qualities and skills. It has been proposed that there is a current paradigm shift in psychology from a field primarily concerned with pathology and what is "wrong" with the person, to a new model of psychological health, thriving, wellness enhancement, and human strengths and growth (Lechner, Zakowski, Antoni, Greenhawt, Block \& Block, 2003; Tedeschi \& Kilmer, 2005). However, in order for this approach to be put into clinical practice, more research is needed that speaks to its efficacy.

It is important for medical doctors, health care providers, therapists, and psychologists to understand the possibility and process of PTG in cancer survivors. In particular, it is important to determine how anxiety, depression, perceived threat, and cognitive processing are associated with growth. This knowledge will allow a more whole and balanced view of responses to cancer. It will also aid in the development of 
clinical interventions that draw upon and enhance individuals' strengths and skills, which can be utilized in the adjustment to illness.

Yet, health providers must be cautious not to prescribe growth to a survivor, minimize the psychological distress that usually accompanies cancer, or suggest that those who do not experience PTG are somehow deficient. Until researchers understand more about the psychological origins of growth, the conditions under which growth occurs, and the best ways to assess growth, designing clinical interventions aimed at enhancing growth may be premature (Park \& Helgeson, 2006). The presence of PTG may not necessarily be accompanied by greater well-being and less distress. Nonetheless, research suggests that the occurrence of PTG is an indication that persons who experience it perceive that they are living life in a fuller and more meaningful way than they had before the traumatic event (Calhoun \& Tedeschi, 2006).

The overall purpose of this study was to examine how cognitive processing is related to PTG in breast, prostate, and colorectal cancer survivors. Second, in order to understand factors that influence cognitive processing, this study examined how symptoms of anxiety and depression, as well as perceived threat, are associated with cognitive processing.

\section{Importance of Studying the Problem}

The possibility of growth following a struggle with highly stressful events fits well with emerging models of therapy that emphasize strength and resilience. This growing body of research has produced many interesting findings, but also has raised unanswered questions about the specific mechanisms related to the emergence of PTG. As mentioned previously, one model of the etiology of PTG proposes that cognitive 
processing rebuilds beliefs after trauma has violated an individual's basic beliefs about the self and the world. Thus, growth is thought to emerge from a period of inquiry in which one attempts to make sense of a traumatic event, including its causes and implications (Park \& Helgeson, 2006). Yet, much is still unknown about the relationship between cognitive factors and PTG. For instance, more research is needed on how the valence and content of cognitions relate to growth. Additionally, more research is needed to clarify some of the mechanisms by which growth experiences occur.

This research is important for several reasons. First, it will increase understanding of cancer survivors' capacity to not only withstand the hardships and obstacles that come with illness, but to actually transform and grow as a result of cancer. Such an understanding may enlarge the traditional focus of psychology on "problems" and contribute to the development of effective interventions that seek to facilitate growth and thriving among those who deal with challenging illnesses.

Second, the processes through which PTG occurs remain vague. The current study will attempt to clarify the relationship between symptoms of anxiety, depression, perceived threat, and cognitive processing in predicting PTG. The results of this study may have important implications for cancer survivors in terms of life purpose and meaning.

Third, research has suggested that PTG may be associated with improved physical health. For example, PTG has been associated with decreases in pain in a mixed sample of cancer and lupus patients (Katz, Flasher, Cacciapaglia, \& Nelson, 2001). While the relationship between these variables is presently unclear, if health care providers understand the factors that may lead to growth efforts to increase PTG can be 
implemented. These interventions might have the potential to buffer against poor physical health.

Fourth, this study may have implications for psychologists, as well as other healthcare professionals. By gaining a greater understanding of the potential for PTG in the aftermath of cancer, psychologists may gain a broader awareness of the possible psychological responses following major medical illness. Greater awareness of PTG may, in turn, lead psychologists to adopt a more nuanced approach with survivors, which includes listening for the growth survivors may be experiencing and focusing on aspects of PTG as they emerge in sessions.

\section{Overview of Hypotheses}

1. Anxiety, Depression, and Perceived Threat will predict Positive Cognitive Processing over and beyond the contribution of the demographic control variables. It is hypothesized that Anxiety and Depression will negatively predict Positive Cognitive Processing, while Perceived Threat will positively predict Positive Cognitive Processing.

2. Positive Cognitive Processing will significantly predict PTG over and beyond the demographic control variables, Anxiety, Depression, and Perceived Threat. In other words, Positive Cognitive Processing will account for a significant amount of the variance of PTG beyond the demographic control variables (i.e., Age, Gender, Stage of Diagnosis, Time Since Treatment Completion), Anxiety, Depression, and Perceived Threat. It is hypothesized that Positive Cognitive Processing and Perceived Threat will positively predict PTG, while Anxiety and Depression will negatively predict PTG. 
3. Anxiety, Depression, and Perceived Threat will predict Negative Cognitive Processing over and beyond the contribution of the demographic control variables. It is hypothesized that Anxiety, Depression, and Perceived Threat will all positively predict Negative Cognitive Processing.

4. Negative Cognitive Processing will significantly predict PTG over and beyond the demographic control variables, Anxiety, Depression, and Perceived Threat. In other words, Negative Cognitive Processing will account for a significant amount of the variance of PTG beyond the demographic control variables (i.e., Age, Gender, Stage of Diagnosis, Time Since Treatment Completion), Anxiety, Depression, and Perceived Threat. It is hypothesized that Negative Cognitive Processing, Anxiety, and Depression will negatively predict PTG, while Perceived Threat will positively predict PTG.

\section{Overview of Variables and Measures}

Several demographic factors were included in the analyses as control variables (Age, Gender, Disease Stage at Diagnosis, Time Since Treatment Completion), which helped lessen potential confounds as described in the literature. The independent variables in the first and third hypotheses were Anxiety, Depression, and Perceived Threat of cancer. Positive and Negative Cognitive Processing were the dependent variables, respectively. In the second and fourth hypotheses, the independent variables were Anxiety, Depression, Perceived Threat, Positive Cognitive Processing (Hypothesis 2), and Negative Cognitive Processing (Hypothesis 4). PTG was the dependent variable.

A demographic questionnaire was used to collect background information as well as cancer diagnosis and treatment information, which were used in statistical analyses. No identifying information was collected. Perceived Threat was broken into three 
components: Life Outlook (i.e., the extent to which cancer challenged the way the survivor sees him/herself, others, and the world), Physical Threat (i.e., mortality salience), and Reaction to Cancer (i.e., reacting with feelings of fear, helplessness, or horror), and was measured by asking participants several questions. Examples of questions are: "To what extent did being diagnosed with cancer challenge the way you see the world in general?" and "At that time [time first diagnosed], how likely did you think it was that you would die of cancer?" These questions were answered on a Likert scale ranging from $1=$ not at all to $6=$ extremely. The Hospital Anxiety and Depression Scale, developed by Zigmond and Snaith (1983), was used to measure symptoms of anxiety and depression. The Cognitive Processing of Trauma Scale (Williams, Davis \& Millsap, 2002) was used to measure cognitive processing of cancer. Finally, the Posttraumatic Growth Inventory, developed by Tedeschi and Calhoun (1996), was used to measure PTG following cancer. Each measure mentioned above is a self-report instrument, and based upon piloting the entire questionnaire with five cancer survivors, it was estimated that completion of all measures would take approximately 20-30 minutes.

\section{Limitations}

Several limitations of this study should be noted. First, the study tested the above hypotheses in a sample of breast, prostate and colorectal cancer survivors, thereby limiting the generalizability of the results to other cancer survivors, or trauma survivors, as a whole.

Second, the scores on the self-report measures used in this study are based upon participants' perceptions. Scores on self-report perceptual measures are often biased and vulnerable to socially desirable responding (response bias). Yet, using a survey with self- 
report measures was the most efficacious way to collect the data for the current study.

Effort was made to select measures that use non-leading questions, have established validity and reliability, and have been used for previous research in the PTG and/or cancer literature. However, the Cognitive Processing of Trauma Scale (2002), while it met the needs of this study, was developed fairly recently, and thus has not been widely used. Moreover, the variable of perceived threat is theoretically based upon the model of PTG, and therefore was measured by response items created specifically for this study, rather than on an empirically tested assessment measure - the reliability and validity of these items is not yet established.

Third, another potential limitation is the sample size due to a lack of participant interest, motivation, fatigue, or other side effects due to illness. Effort was made to make the data collection portion of this study brief for participants (20 - 30 minutes) in order to lessen response burden and time demands.

Fourth, using a cross-sectional, retrospective research design prevents the drawing of conclusions about a causal and temporal relationship between the independent and dependent variables, and is susceptible to recall biases. Experimental and longitudinal research designs that assess variables pre and post cancer diagnosis are needed to address this limitation; however, these designs are difficult to implement as participants are typically identified after a cancer diagnosis has already occurred. Despite these limitations, this study represents an important step in clarifying the relationship between cognitive processing and PTG, and how perceived threat, anxiety, and depression contribute to these factors. 


\section{Summary}

Cancer survival has increased due to advances in medicine; yet, large numbers of people are still diagnosed with cancer. The diagnosis of cancer is potentially life threatening, and initial diagnosis, along with subsequent treatment, is often a shocking and traumatic event. Having cancer has the potential to evoke a wide range of psychological reactions. Literature shows that many of these reactions are negative, including, for some, evoking clinically significant levels of depressive and anxiety symptoms. However, over the past decade research has also suggested that the struggle to deal with traumatic events, such as cancer, may result in positive changes. Examples of such changes are positive views about oneself and one's relationships. Tedeschi and Calhoun (1995) coined the widely used term posttraumatic growth, to refer to the spectrum of positive changes an individual may experience after a traumatic event (Manne et al., 2004).

Distress in the aftermath of a cancer diagnosis is likely to be a common experience for many, and an individual's perception of PTG does not necessarily decrease or buffer against distress. Nonetheless, research has shown that the struggle with cancer often leads to changes, which some individuals regard as highly positive. PTG may lead a person to believe he/she is living a richer and more purpose-filled life, and has the ability to create possibilities and potentials that might not have been imaginable before cancer. The empirical research on PTG is growing and offers promising findings. A better understanding of the process of PTG will help to clarify and further research. This study will specifically focus on the role of cognitive processing, and how factors 
often related to the cancer experience (perceived threat, anxiety, depression) influence this process.

Chapter One provided the background of the concept of PTG following cancer. This chapter also included a statement of the problem, purpose of studying the problem, importance of studying the problem, hypotheses, overview of the variables and measures associated with the study, and limitations of the study. Please refer to Appendix A for a glossary of terms used in the study. Chapter Two will present a review of the literature relevant to this study as well as the theoretical basis of the hypotheses outlined above. 


\section{CHAPTER TWO}

\section{REVIEW OF SELECTED LITERATURE}

\section{Introduction}

Throughout time, across many different religious and philosophical traditions, there has been an understanding that suffering and hardship have the ability to produce positive outcomes (Calhoun $\&$ Tedeschi, 2006). There is often a perception among those who have endured tragedy that they have been strengthened in some form or fashion by their struggle with it. An event that is initially extremely aversive may lead to psychological transformation and growth. People may not feel pleased that trauma has occurred, but research has discovered that many people view the aftermath of trauma as something that has benefited them (Tedeschi \& Calhoun, 1995).

Individuals' reactions to trauma are very important to study as they offer insights into human nature, and the capability humans hold to not only survive, but grow in the face of suffering. In classic literature, heroes are often portrayed as "tragic figures" who, after struggling with whatever tragedy befalls them, emerge as their "best selves" (Tedeschi \& Calhoun, 1995). This notion is not just reserved for literature, but rather, seems to hold true for many "ordinary" people who achieve "extraordinary" outcomes 
when faced with trauma. The experience of trauma can be very terrible and upsetting, yet it can also lead to what may come to be regarded, in the extreme, as "the best thing that ever happened to me." Trauma can also be regarded simply as a beneficial experience, by providing the opportunity for psychological growth that would not be possible without the challenge of the traumatic event. It is the very act of struggling with the consequences of trauma that makes possible varied forms of PTG (Tedeschi \& Calhoun, 1995).

Over the past decade, researchers have moved beyond an exclusive focus on the negative consequences following traumatic events (Linley \& Joseph, 2004; Park \& Helgeson, 2006; Wortman, 2004; Zoellner \& Maercker, 2006). There is now a growing body of research that documents positive ways that people's lives have changed following trauma. Studies on PTG are important for several reasons. Most notably, focusing only on the negative outcomes of trauma can lead to a biased understanding of posttraumatic reactions. Traditionally, little effort has been made to understand how the worldviews and identities of survivors have been altered in the aftermath of trauma. Instead, concentration has rested on fairly routine procedures of symptom reduction (Ballou \& Brown, 2002).

An understanding of posttraumatic reactions needs to take into account the potential for positive, as well as negative changes, if it is to be comprehensively studied (Linley \& Joseph, 2004). When considering the implications and consequences of PTG, it is important to realize that the phenomenon is not limited to a particular type of trauma. Positive changes have been empirically reported following circumstances such as chronic illness, heart attacks, cancer, bone marrow transplants, HIV and AIDS, rape and sexual assault, military combat, bereavement, and natural disasters (Linley \& Joseph, 2004; 
Milam, 2006; Park \& Helgeson, 2006; Paton, 2006; Rosner \& Powell, 2006; Wortman, 2004; Znoj, 2006). Therefore, PTG is potentially the result of numerous factors, and there is a present need to establish more clearly through research the variables that are associated with growth in the aftermath of trauma.

In order to effectively address the potential for PTG, it is important to explore, and subsequently more fully understand, the factors contributing to responses after trauma. This literature review will provide a rationale for the relationships suggested in the proposed model in this study. The theoretical framework for the study comes from previous studies conducted with people who have experienced a variety of traumatic events. This study will focus specifically on individuals who have survived breast, prostate, and colorectal cancer.

Why Cancer as a Model of PTG

According to Geffen (2006), "Cancer is a growing presence in our society" (p. 20). The author explained that, "If heart disease was the affliction of the World War II generation, cancer is the disease of the baby boomers and more and more people...ready or not, are suddenly being forced to confront it" (p. 20). Breast, prostate and colorectal cancer, along with lung cancer, comprise the leading anatomical sites for new cancer cases, as well as cancer deaths, in the United States (American Cancer Society, 2008). The overall number of new cancer diagnoses continues to increase each year (Geffen, 2006) and the cumulative impact of these cancers on society is enormous.

Cancer is a disease that has the ability to strike fear in many individuals and often creates a great deal of uncertainty, suffering and loss (Lechner \& Weaver, 2009). On being told that one has cancer, a sense of panic often sets in. Lechner and Weaver (2009) 
explained that many survivors report that they do not recall much of the conversation with their physician after hearing the news. In the researchers' work, they found that receiving a diagnosis of cancer is a "seismic event" (Tedeschi \& Calhoun, 2004), and this "earth-shattering news" often causes a rush of emotions and cognitions. In the following days, weeks, and months survivors confront hardships and struggles that result from both the illness and its treatment. Yet, paradoxically, Lechner and Weaver (2009) found that many survivors experience positive life changes.

Park (2009) explained that medical illnesses, including cancer, "set the stage for the kind of existential confrontations and global violations of beliefs and goals that are thought to lead to meaning making and ultimately to growth, thus leading researchers to look in this direction" (p. 21). The impact of cancer is widespread, and as Park (2009) described, a better understanding of growth in the context of medical illness may have implications for clinical interventions, even at the broad level of public health, where knowledge gained from research could have a substantial impact. However, a major limitation in the existing PTG literature is a lack of understanding of the process of, and a clearly defined set of variables related to, growth. The current study aimed to fill in gaps in the research by examining the effect of cognitive processing on PTG in breast, prostate and colorectal cancer survivors. In addition, this study determined how symptoms of anxiety and depression, as well as perceived threat, affected cognitive processing, which in turn, predicts PTG.

This chapter includes an examination of PTG, providing an overview of common challenges related to the topic, and the relationship between cancer and growth. This chapter also explores how cognitive processing relates to PTG and reviews the most 
relevant literature regarding theoretically derived predictors of PTG, including perceived threat, anxiety, and depression.

\section{Trauma}

In order to understand and appreciate the concept of PTG, one first must have a general understanding of what constitutes trauma, and the negative consequences of such an event. According to the American Psychiatric Association Diagnostic and Statistical Manual of Mental Disorders, $4^{\text {th }}$ Edition, Text Revision, an "extreme traumatic stressor" involves

direct personal experience of an event that involves actual or threatened death or serious injury, or other threat to one's physical integrity; or witnessing an event that involves death, injury, or a threat to the physical integrity of another person; or learning about unexpected or violent death, serious harm, or threat of death or injury experienced by a family member or other close associate. (DSM-IV-TR, American Psychiatric Association, 2000, p. 463)

Moreover, the person's response to the event must involve "intense fear, helplessness, or horror." Posttraumatic stress disorder (PTSD) is a possible psychological outcome after trauma. The characteristic symptoms resulting from exposure to trauma include persistent re-experiencing of the event, persistent avoidance of stimuli associated with the trauma, “numbing of general responsiveness," and persistent symptoms of increased arousal (APA, 2000).

The word "trauma" indicates that an event was a shock. Researchers have proposed that certain characteristics make events traumatic. These include: an event occurs suddenly and unexpectedly; there is a perceived lack of control over it; the event is out of the ordinary; and the degree to which it creates long-lasting problems (JanoffBulman, 1992; Tedeschi \& Calhoun, 1995). Negative events often have highly negative psychological consequences. Individuals who have experienced a traumatic event might 
endure effects on their thoughts, emotions, behaviors and physical health. For many, thoughts, images, and recollections of the event regularly intrude into conscious awareness, and are most likely unwanted and uninvited (Tedeschi \& Calhoun, 1995). Trauma-related thoughts also impact how individuals think about themselves, and their self-esteem. Being exposed to highly negative events may decrease self-esteem, at least in the first days or weeks after the trauma. Perhaps the most significant way thoughts are affected is the struggle to achieve an understanding of what has occurred. Trauma survivors might ask themselves: "Why did this happen?" and "What was the reason for, or purpose, of this tragedy?" Researchers have suggested that whether or not trauma represents a negative psychological event depends in part on the survivor's satisfaction with how he/she addresses and answers these questions. The process to understand why a crisis has happened, what some researchers have termed the process of "meaning making" (Neimeyer, 2001), is thought to be a common occurrence after trauma. In a 2010 review of the literature on meaning-making, Park (2010) summarized,

It is clear that meaning-making attempts and meanings made are reported by most individuals facing highly stressful events. In fact, it seems logical that some sort of cognitive readjustment or meaning-making process must occur following experiences of events that are greatly discrepant with one's larger beliefs, plans, and desires. (p. 290)

Drawing on clinical experience in working with the bereaved, and research related to bereavement, Schwartzberg and Halgin (1991) reported that after a death, individuals often strive to make sense of the loss and ask questions regarding why the event happened. They suggested that questions such as these pose a severe cognitive threat because they trigger how a person's implicit beliefs about justice and control have failed to explain the profound event. Echoing other grief researchers, the authors explained that 
some people never find meaning while others may develop a way to make meaning out of the loss. Schwartzberg and Halgin (1991) proposed that those who find an answer to "why" questions are attempting to preserve the assumptions that guided their beliefs before the loss, and therefore, in turn, minimize the impact of the trauma. Furthermore, the authors suggested that most grieving individuals experience changes in beliefs about themselves and the world. These include: "reprioritizing values, gaining wisdom, shedding the illusion of immortality, developing a less optimistic view of the world, and questioning spiritual beliefs" (p. 242). Some of these changes are what some researchers term growth.

Tedeschi and Calhoun (1995) suggested that initially ruminative processes attempt to establish comprehensibility. This process occurs when a person tries to grasp the reality of what has happened. The authors proposed that when fundamental understandings of personal reality are violated, there seems to be a time lag between the event and a full appreciation that circumstances are changed. With comprehensibility comes a better ability to manage the trauma and figure out methods to cope. The more reflective element of cognitive processing is what is termed "meaning making," which is thought to occur when an individual has had some success coping with the trauma and is not preoccupied with mere survival. The person is able to move beyond survival to recognition of other possibilities and changes that occurred in the aftermath of the trauma.

It is important to note that searching for meaning in loss is not necessarily related to better adjustment post-trauma. Davis, Wortman, Lehman and Silver (2000) explained that some individuals may never initiate a search for meaning regarding the purpose of a 
trauma, and these individuals appear to adjust relatively well to the loss. Yet, greater distress may occur for a long time after a loss for individuals who search for meaning and never find it. Davis, et al. (2000) described examples of individuals who are at a higher risk to initiate a search for meaning and not find any, and in turn, experience distress. These include individuals who exhibit symptoms of intense anguish and struggle to find meaning shortly after a loss, and people who experience a loss under traumatic circumstances (e.g., sudden, untimely, violent).

The specific emotions people experience in the aftermath of trauma will vary among individuals and across particular circumstances. One emotion that many survivors experience is guilt, resulting from a feeling of remorse about what was done or what was left undone. Feelings of guilt may reflect a belief among individuals that certain actions may have prevented the event, or that certain actions would have made the loss more manageable (Hodgkinson \& Stewart, 1998; Tedeschi \& Calhoun, 1995). Other common emotional reactions to trauma include: anger, irritability, fear, and anxiety; fear and anxiety are frequent emotional responses. It has been suggested that when the circumstances of trauma involve significant threat to life, health, or to important property, it is likely that those individuals will feel apprehension, worry, and concern. Depression is also quite common. While anxiety is more likely to occur when trauma involves major threat, depression is more likely when the circumstances involve significant loss (Tedeschi \& Calhoun, 1995). In general, individuals exposed to highly stressful events are very likely to experience a variety of distressing emotions.

Survivors who are struggling to cope with trauma may also exhibit negative changes in their behavior including: increase in legal and illegal drug use, withdrawal 
from others, sexual difficulties, and an increase in aggressive behavior. Physical problems and complaints are also common in the aftermath of trauma. Individuals exposed to highly traumatic events that occur unexpectedly will often experience an increase in physical arousal. For many, this increase in activation will not quickly subside, and is one of the most common complaints associated with highly stressful events (Hodgkinson \& Stewart, 1998). Because of this chronic arousal of bodily systems, it is not surprising that people report fatigue as well as a long list of other physical complaints, such as gastrointestinal difficulties, headaches, and loss of appetite, etc. (Hodgkinson \& Stewart, 1998). Finally, data also suggest that exposure to stressful events puts a person at increased risk for developing psychiatric disorders (Rubonis \& Bickman, 1991).

However, it is necessary to keep in mind that most people who are exposed to high levels of stress will not develop psychiatric disorders, and that there are many factors that contribute to the development of such disorders, only one of which is exposure to traumatic events. For those who do develop psychiatric disorders, the most likely impairments are anxiety disorders or clinical depression (Goenjian, Steinberg, Najarian, Fairbanks, Tashjian, \& Pynoos, 2000). Many survivors will experience a reduction of psychological distress over time (Lepore \& Revenson, 2006). Yet, it is not time itself that decreases distress, but certain experiences and coping mechanisms used when dealing with the aftermath of trauma. For example, the traumatic event may be reviewed in a person's mind numerous times before the reality of the trauma is accepted and distress is relieved (Tedeschi \& Calhoun, 1995). The available information points to the reality of the negative consequences of trauma, and of the capability of these events to 
greatly test individuals' ability to cope. Yet, the other side of this struggle is that it can lead to the potential for psychological growth that would not be possible without the challenge of trauma.

\section{Trauma and Assumptive Beliefs}

While varying in specifics, most models of growth (Janoff-Bulman, 1992; Taylor, 1983; \& Tedeschi \& Calhoun, 2004) hypothesize that the experience of a traumatic event violates an individual's basic beliefs about the self and the world, and that some type of cognitive processing to rebuild beliefs and goals occurs, resulting in perceptions of growth (Park \& Helgeson, 2006). Thus, there is a period of "psychological reorganization" in the aftermath of trauma. An individual's appraisal of the extent to which events or situations violate his/her beliefs, expectations and goals determine the stressfulness of an event (Park, 1998).

Cognitive processing models of post-trauma reactions propose that people enter situations with preexisting mental schemas. These schemas contain information about individuals' past experiences as well as their beliefs, assumptions, and expectations regarding future events (Creamer, Burgess \& Pattison, 1992). Life is comprehensible because individuals create order through their belief systems, which provide expectations about themselves and the world, which, in turn, allow people to process information and act and respond to events with confidence, or not.

Janoff-Bulman and Frieze (1983) explained that people typically operate on the basis of assumptions and personal theories that allow them to set goals, plan activities, and order their behavior. The authors proposed that there are three core assumptions that form the basis for many individuals' "assumptive worlds:" seeing ourselves as having 
control over events and being relatively invulnerable to harm; viewing the things that happen to us as orderly, predictable, and meaningful; and regarding ourselves and others in a positive light (Janoff-Bulman \& Frieze, 1983). Without a set of fundamental beliefs individuals would not be able to make sense of the world around them, and, therefore, a person's assumptive world is generally resistant to change. Yet, the occurrence of a traumatic event often represents what is unknown and incomprehensible, and confronts people with information that is inconsistent with that contained in existing schemas about their safety and invulnerability (Creamer et al., 1992). Thus, a traumatic event has the ability to shatter the understanding that people have of the world.

Janoff-Bulman (2004) suggested that as non-victims, individuals believe they are prepared for misfortune. At a rational level, people know that car accidents are common, cancer strikes large percentages of people, and crime is widespread, but at some deeper, experiential level we do not seem to accept it. The author explained that while people know bad things happen, they do not believe it will happen to them, and therefore they are psychologically unprepared for trauma. When confronted with trauma, people face threatening questions and prospects, such as death, which are a part of the human experience, but usually hidden out of conscious awareness. The internal world of an individual is thrown into upheaval because the assumptions that provided psychological stability and coherence are now viewed as inadequate and inaccurate in describing the posttraumatic world of the survivor (Janoff-Bulman, 2004).

Horowitz (1986) argued that for recovery from trauma to occur, new information inherent in the traumatic experience must be processed until it can be brought in line with personal schemas. Yet, attempts to assimilate threat-related information may be avoided 
by a survivor, as assimilation requires exposure to aversive stimuli, which often results in increased arousal and desire to avoid thoughts and reminders of the trauma. Creamer et al. (1992) asserted that until a traumatic event can be incorporated and integrated into existing schemas, it is stored in active memory, and the psychological elements of the event continue to produce intrusive and emotionally upsetting thoughts and/or recollections. Yet, preexisting schemas often do not accurately reflect a survivor's life after trauma and, therefore, modification of pre-trauma schemas typically occurs to accommodate new information. The questioning of basic assumptions is what prompts personal change through the task of rebuilding an assumptive world that accommodates the reality of the experienced trauma.

Janoff-Bulman (1992, p. 133) explained, "By engaging in interpretations and evaluations that focus on the benefits and lessons learned, survivors emphasize benevolence over malevolence, meaningfulness over randomness, and self-worth over self-abasement." By working to rebuild their fundamental assumptions, individuals incorporate the data of their experience and new, and perhaps more negative, assumptions are formed that acknowledge the survivor's greater danger and increased vulnerability. While negative views may pervade the inner world of survivors in the immediate aftermath of the trauma, Janoff-Bulman (2004) proposed that over time survivors reestablish generally positive, yet less absolutely positive, core assumptions.

For some the process of rebuilding assumptions and beliefs is more difficult, and in some cases posttraumatic stress disorder (PTSD) might develop after a trauma. Foa and Riggs (1993) and Foa and Rothbaum (1998) proposed that after exposure to trauma two basic cognitive dysfunctions are associated with the development of PTSD: that the 
world is completely dangerous, and that one is totally incompetent. The authors suggested that individuals who encounter a traumatic event with the notion that the world is extremely safe, and they are extremely competent, have more difficulty in assimilating the traumatic experience into their existing schemas. Further, for those who have experienced various traumas throughout their lives, a traumatic event may trigger existing beliefs about the dangerousness of the world and one's abilities. Thus, rigid beliefs about self and the world, positive or negative, may lead to more distress after trauma.

Conversely, the authors suggested that people who have less rigid beliefs, and are able to view a trauma as a unique event that does not have broad implications about self and the world, will likely experience less distress and would not develop PTSD (Foa \& Riggs, 1993; Foa \& Rothbaum, 1998).

\section{Posttraumatic Growth}

\section{Overview}

Taylor (1983, p. 1161) argued, "One of the most impressive qualities of the human psyche is its ability to withstand severe personal tragedy successfully." In addition to the negative outcomes of trauma mentioned above, many trauma survivors also experience positive psychological changes. Zoellner and Maercker (2006, p. 628) defined PTG as "the subjective experience of positive psychological changes reported by an individual as a result of the struggle with trauma." Examples of positive psychological change include: an increased appreciation of life, setting of new life priorities, a sense of increased personal strength, identification of new possibilities, improved closeness of intimate relationships, or positive spiritual change (Tedeschi, Park, \& Calhoun, 1998). 
PTG describes the experience of individuals who not only recover from trauma, i.e., return to pre-trauma level functioning after a period of emotional distress, but use the trauma as an opportunity for further individual development. Individuals who have experienced PTG overcome trauma with improved psychological functioning in specific areas. For example, an individual who is confronted with cancer might experience a shift in priorities that results in spending more time with loved ones (Zoellner \& Maercker, 2006).

Many terms have been used in the literature to describe the phenomenon of growth (finding benefits, stress-related growth, thriving, positive psychological changes, adversarial growth). This study uses the term "posttraumatic growth" as defined by Tedeschi and Calhoun $(1995,2004)$ as it best captures the meaning of the phenomenon. The term "posttraumatic" implies that the growth happens in the aftermath of a highly stressful/traumatic event, not as the result of any minor stress or as a part of a natural developmental process. The term "growth" emphasizes that the person has developed beyond his/her previous level of adaptation, psychological functioning, or life awareness (Zoellner \& Maercker, 2006). In other words, there is an additional benefit compared to the pre-crisis level.

Posttraumatic growth refers to a change in people that goes beyond an ability to resist and not be damaged by highly stressful events; it involves a movement beyond pretrauma levels of adaptation. Posttraumatic growth, then, has a quality of transformation, or a qualitative change in functioning. (Tedeschi \& Calhoun, 2004, p. 4)

PTG has been conceptualized as both an outcome of the struggle with a traumatic event (Schaefer \& Moos, 1992; Tedeschi \& Calhoun, 1995, 2004), and as a coping strategy (Affleck \& Tennen, 1996). This study conceptualized PTG as an outcome, 
utilizing the model proposed by Tedeschi and Calhoun $(1995,2004)$. One of the reasons this model is useful is because it attempts to illustrate the mechanisms of PTG. In the PTG as outcome model, coping influences PTG, but growth is the transformative result of the struggle with trauma rather than a particular coping strategy used to deal with the event.

Schaefer and Moos (1998) explained that environmental and personal factors, such as social support and demographic characteristics, shape the traumatic experience and its aftermath. The authors suggested these factors influence cognitive appraisal processing and coping responses, which, subsequently affect the outcome of trauma and the perception of growth. They emphasized the important role of approach coping, as opposed to avoidance coping, for growth to occur. Approach coping involves attempting to analyze trauma in a logical way, reappraising the crisis in a more positive light, seeking support, and taking actions to solve the problem. Avoidance coping includes trying to minimize the problem, deciding that nothing can be done to change it, seeking alternative rewards, and venting emotions (Schaefer \& Moos, 1998). Thus, they suggested that the specific ways in which an individual copes has an influence on whether or not growth is experienced.

\section{Model of Posttraumatic Growth}

Tedeschi and Calhoun (2004) conceptualized the growth process as follows: a traumatic event, which they describe as an event of "seismic" proportions, shakes or destroys some key elements of an individual's important goals and worldview. It represents a challenge to goals, beliefs, and the ability to manage emotional distress. The resulting emotional distress initiates a process of recurrent rumination and attempts to 
engage in behavior that is intended to reduce distress. Initially, rumination is more automatic than deliberate. It is characterized by frequent returns to thinking about the trauma and related issues. After initial coping success (e.g., reduction of emotional distress, disengagement from unreachable goals, etc.) rumination may evolve to more purposeful thinking about the trauma and its impact on one's life.

In the initial aftermath of a traumatic event, people often do not conceive of positive outcomes, or growth, due to being overwhelmed by distressing emotions and loss. Only through the reduction of some of this distress does growth become possible (Tedeschi \& Calhoun, 1995). Rumination, in the form of constructive cognitive processing (analyzing the situation, re-appraisal, and finding meaning) is assumed to play a critical role in the development of personal growth. PTG is conceptualized as a multidimensional construct including changes in beliefs, goals, behaviors, and identity, as well as the development of a life narrative and wisdom.

Tedeschi and Calhoun (1996) proposed five domains of growth (as measured by the Posttraumatic Growth Inventory (PTGI)): greater appreciation of life and a changed sense of priorities; warmer, more intimate relationships with others; a greater sense of personal strength; recognition of new possibilities or paths for one's life; and spiritual development. Individual characteristics, styles of managing distressing emotions, the degree to which individuals engage in self-disclosure about their emotions and perspective on trauma, cognitive processing (specifically, the process of deliberate ruminative thought), and some degree of enduring distress are all believed to influence the emergence of PTG (Tedeschi \& Calhoun, 2004; see Figure 1). This study focused on one of these elements; the role of cognitive processing in the development of PTG. 
Figure 1: A model of posttraumatic growth.

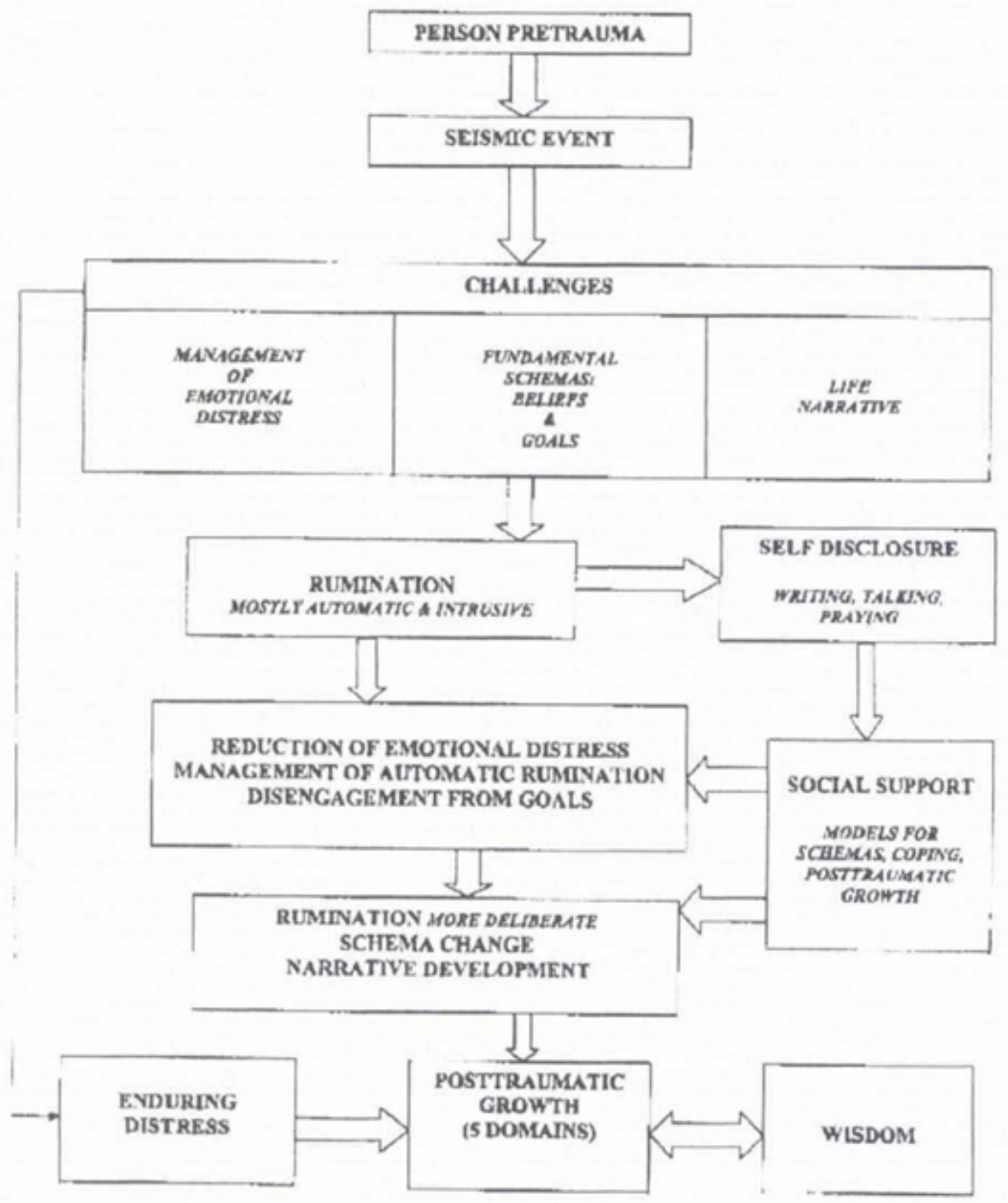

Figure 1. A model of posstruamatic growith.

Note. From Tedeschi, R.G. \& Calhoun, L.G. (2004). Target Article: "Posttraumatic

Growth: Conceptual Foundations and Empirical Evidence." Psychological Inquiry, 15(1), 1-18. 


\section{Challenges to the Concept of PTG}

There are arguably two main controversies in the current PTG literature: (1) whether PTG and related constructs reflect genuine positive change, and (2) whether the manner in which growth is typically measured is valid (Frazier, Tennen, Gavian, Park, Tomich \& Tashiro, 2009). Several researchers have wondered whether perceptions of growth are real or illusory (Frazier, et al., 2009; Helgeson \& Park, 2009; Sumalla, Ochoa \& Blanco, 2009; Wortman, 2004). Wortman (2004) suggested that statements of positive change might represent defensive illusions, such that survivors want to convince themselves that something good has come out of the loss they have endured. Furthermore, the author proposed that individuals are often motivated to depict a more positive view of their lives than is actually the case in order to convey to others that they are coping well (Wortman, 2004).

Yet, illusions are not necessarily negative, nor untrue. Taylor and Brown (1988) argued that positive illusions lead to positive mental health because they add to feelings of well-being, increase optimism in one's choices and future path, promote confidence in one's abilities and enhance self-regard, all of which have been associated with positive psychological outcomes. Moreover, Taylor and Brown (1988) reported that illusions are adaptive when an individual is challenged by adversity, such as a diagnosis with cancer. Evidence from converging sources suggests that positive illusions about the self, one's control, and the future may be especially apparent and adaptive under circumstances of adversity, that is, circumstances that might be expected to produce depression or lack of motivation. Under these circumstances, the belief in one's self as a competent, efficacious actor behaving in a world with a generally positive future may be especially helpful in 
overcoming setbacks, potential blows to self-esteem, and potential erosions in one's view of the future (Taylor \& Brown, 1988, p. 201).

Related to the question of whether reports of PTG are real or illusory, is the question of whether to conceptualize the report of growth as a means of coping (either as an adaptive mechanism or a defensive, self-protective strategy), or as an outcome in and of itself representing true change (Butler, 2007). Helgeson, Reynolds and Tomich (2006) described,

Growth outcomes may reflect a variety of processes, some of which have to do with actual changes in one's life, some of which have to do with coping, and others of which have to do with cognitive manipulations on the order of self-enhancement biases meant to alleviate distress. (p. 812)

As mentioned above, this study will conceptualize PTG as an outcome. One reason it is useful to conceptualize PTG in this manner is because research suggests growth and psychological distress are two independent dimensions of well-being (Park \& Helgeson, 2006).

Cognitive engagement and rebuilding typically takes into account the changed reality of one's life and produces schemas that incorporate the trauma, which may be experienced as growth. Yet, the trauma itself may remain distressing (Tedeschi \& Calhoun, 2004). Often in the literature, distress and growth are conceptualized as having an ipsative relationship, such that if one experiences growth, distress will decrease. However, Tedeschi and Calhoun (2004) argued that distress and PTG are two distinct constructs, and a person can experience them simultaneously.

Baker, Kelly, Calhoun, Cann, and Tedeschi (2008) conducted a study where both PTG and posttraumatic depreciation were measured. The researchers designed a scale that mirrored the Posttraumatic Growth Inventory, but assessed depreciation in life 
domains, and administered both scales to a group of undergraduate students. Results revealed that changes in growth and depreciation were independent. Moreover, the researchers concluded that individuals who experience significant stressors might simultaneously report depreciation in the same areas they report growth (Baker et al., 2008). Cordova and Andrykowski (2003) explained that it is possible to experience cancer-related stress and growth concurrently because individuals often view the experience as both a trauma and a transition into a new chapter in their lives. Thus, growth may best be conceptualized as an outcome of importance in its own right (Park \& Helgeson, 2006).

Another critique of PTG relates to measurement. PTG is most often assessed by self-report, through interviews and/or questionnaires, and some believe these reports are not valid (Frazier \& Kaler, 2006). Ransom, Sheldon and Jacobsen (2008) explained that the validation of survivors' PTG reports has been challenging, largely due to the difficulty in obtaining pre-stressor data from individuals who subsequently survive a traumatic event. Thus, it is largely unknown whether individuals' reports of PTG reflect actual, measurable change from their pre-stressor state.

Two routine measures of growth, the Posttraumatic Growth Inventory and the Stress-Related Growth Scale have sound psychometric properties (Helgeson, Reynolds \& Tomich, 2006); however, these measures may not be sophisticated enough to capture the complexity of growth. Moreover, Helgeson, et al. (2006) explained that it is unclear how these instruments could be altered so that they may be able to distinguish actual growth from perceived growth from coping. The shortcomings of current growth assessment instruments are a limitation of the literature that needs to be taken into consideration. 
Future research also needs to examine growth using multiple methods at various time points, and to examine the process of growth, which is likely multi-faceted. This study seeks to examine variables, such as cognitive processing, which will aid in understanding how PTG emerges after trauma.

Cognitive Processing and Posttraumatic Growth

Affleck and Tennen (1996) suggested that cognitive models of trauma all share the premise that trauma can lose some of its subjective severity through cognitive adaptations. One of these adaptations, for example, might be finding the "good in bad events," which can restore encouraging views of ourselves, other people, and the world. Research on the correlates of PTG is consistent with the notion that cognitive processing of the traumatic event has an important role in facilitating growth. It has been proposed that individuals who cope effectively with traumatic events actively contemplate and process their experience using cognitive, affective, and interpersonal processing strategies (Manne, Ostroff, Winkel, Goldstein, Fox \& Grana, 2004). A number of indicators of cognitive processing (e.g., rumination, intrusions, positive appraisal, acceptance) have been found to relate to PTG (Helgeson, Reynolds \& Tomich, 2006; Linley \& Joseph, 2004).

In line with the assumptive world literature above, Carboon, Anderson, Pollard, Szer and Seymour (2005) asserted that the potential for growth is somewhat dependent on the degree of positive bias in an individual's pre-trauma assumptions and therefore the degree to which assumptions are challenged by the event. Likewise, the theoretical framework used in this study (Tedeschi \& Calhoun, 2004) proposed that the degree to which a traumatic event is perceived as threatening by an individual (i.e., challenges 
his/her fundamental beliefs and is perceived as a threat of death or physical integrity), and is actively and deliberately thought about, will influence the amount of PTG experienced. Therefore, deliberate rumination plays a significant role in the process of growth.

For instance, Bower, Kemeny, Taylor, and Fahey (1998) conducted a study with 40 HIV-positive men who had recently experienced an AIDS-related bereavement. The results indicated that those men who engaged in active or deliberate thinking about the death were more likely to report positive shifts in their values or priorities in response to the loss. The authors explained that actively thinking about trauma can be a painful process, provoking short-term increases in negative mood and certain measures of autonomic activity. However, this process may be needed to reach a positive cognitive outcome following the event, creating changes in attitudes and values that would not otherwise be conceived.

When considering the role of cognition, and more specifically rumination, in the facilitation of growth, it is necessary to keep in mind that there is a lack of consistency in the use of the term "rumination" in the psychology literature, and it can be either adaptive or maladaptive depending on the particular definition used. For instance, when rumination is defined as "persistent thoughts about one's symptoms of distress, and the possible causes and consequences of these symptoms" (Nolen-Hoeksema \& Davis, 2004, p. 62), it is associated with more distress, more negative thinking, poorer problem solving, and other negative outcomes. In contrast, "reflection," defined as analyzing recent events in order to increase understanding, has been found to be associated with benefit finding and forms of coping that seem likely to be associated with PTG (Nolen- 
Hoeksema \& Davis, 2004). This study utilized Martin and Tesser's (1996) general definition of rumination as "a class of conscious thoughts that revolve around a common instrumental theme and that recur in the absence of immediate environmental demands requiring the thoughts" (Martin \& Tesser, 1996, p. 1).

Intrusive thoughts, which are common and automatic initially following a traumatic event, are typically categorized in the literature as maladaptive and associated with avoidance and escape. Furthermore, attempts at avoidance, particularly of traumarelated thoughts and feelings, have been conceptualized as key to the development and maintenance of PTSD. Yet, some researchers have argued that intrusive thoughts are adaptive. Individuals who have experienced a traumatic event tend to think about it frequently in an attempt to understand, resolve, and make sense out of what happened. Horowitz (1986, p. 99) explained that intrusive thoughts may lead to "revising the automatic processing of such information, to revising the relevant schemas... and to completing the processing of the stressful information." Creamer, et al. (1992) argued that escape and avoidance may reduce immediate distress, but long-term reliance on these methods might be maladaptive. The authors explained that for recovery to occur, memories must be activated for periods long enough to allow for effective processing, which tends not to occur when escape and avoidance are high. Examples of effective processing identified in the literature include: talking about the trauma with family and friends, therapeutic exposure to trauma-related stimuli, and deliberate attempts to access new information. Creamer et al. (1992) explained that in these instances the thoughts and memories may not be intrusive, but rather individuals are making a conscious effort to recall the event. 
Research has suggested that cognitive processing is related to the amount of PTG individuals' report (Gangstad, Norman \& Barton, 2009; Phelps, Williams, Raichle, Turner \& Ehde, 2008; Salsman, Segerstrom, Brechting, Carlson \& Andrykowski, 2009). Research has also indicated that the timing of cognitive processing is significant. For example, when intrusive thoughts are actively processed early in the cancer experience rather than ignored, they are likely to result in a better psychological outcome (Manne et al., 2004). Calhoun, Cann, Tedeschi, and McMillan (2000) conducted a study with 54 individuals who had experienced a traumatic event, and results indicated a relationship between event-related rumination and the amount of PTG reported. The more rumination participants experienced soon after the event, the greater the amount of PTG. The authors concluded that early event-related rumination is associated with growth. Moreover, it is expected that people who purposefully think about the event, and its potential meaning and importance, are more likely to report PTG. However, the authors also speculated that when ruminations are primarily intrusive, negative, and continue consistently for long periods of time, both low levels of growth and high levels of distress would be expected.

Salsman, Segerstrom, Brechting, Carlson, and Andrykowski (2009) conducted a study with 55 post-treatment, colorectal cancer survivors, which demonstrated the role cognitive processing might have not only on growth, but on distress. As part of the study, participants completed measures assessing cognitive processing (cognitive intrusions, cognitive rehearsal) and psychological adjustment variables, including PTSD and PTG. It was found that baseline intrusive thoughts were not significantly associated with baseline or 3-month PTGI scores. Also, baseline cognitive rehearsal (defined as a form of deliberate cognitive processing) was neither predictive of baseline PTGI scores nor 
predictive of 3-month PTGI scores. After controlling for baseline PTGI scores, and excluding both age at diagnosis and education, baseline cognitive rehearsal was found to predict 3-month PTGI scores (Salsman et al., 2009).

The researchers concluded that in contrast to the lack of associations between automatic, intrusive cognitions and PTG, more intentional effortful processing was weakly associated with higher levels of growth. Though baseline cognitive rehearsal was not associated with baseline growth, the relationship between baseline cognitive rehearsal and 3-month PTG suggested a trend. Moreover, the researchers hypothesized that individuals who were experiencing higher levels of intrusive thoughts, along with PTSD symptoms, were also engaging in more deliberate reflection as a means to begin processing their cancer experience. Three months later, those who had engaged in higher levels of cognitive rehearsal were less likely to report higher levels of PTSD symptoms and more likely to report higher levels of PTG (Salsman et al., 2009). Thus, based on these results, it appears that cognitive rehearsal is positively associated with PTG.

Finally, the important role of cognitive processing in PTG is not meant to imply that a survivor's emotions are not important. Yet, research has suggested that more growth may occur when both emotions and thoughts are processed. Ullrich and Lutgendorf (2002) reported that emotional expression, focused specifically on cognitive processing following exposure to trauma (i.e., writing in a journal about emotions and making sense of the traumatic event) was related to increased positive growth over a one month time period. The authors suggested that engagement of both cognitions and emotions while journaling about a traumatic event can raise awareness of the benefits of the event. In contrast, focusing solely on the emotions related to the event might not 
produce a greater understanding of the trauma. Furthermore, they explained that creating a coherent explanation for the event may help restore self-efficacy and add meaning to the event (Ullrich \& Lutgendorf, 2002). In general, the above findings support the notion that the greater the opportunity to contemplate stressor-related thoughts and feelings, the greater the opportunity for PTG.

\section{Unique Aspects of the Cancer Experience}

Sumulla et al. (2009) suggested five ways that cancer is different than an acute trauma, such as a car accident, and therefore is somewhat unique. First, whereas some traumatic events can be characterized by a specific and single stressor, during cancer the stressors may be associated with "the diagnosis of cancer, its severity and prognosis, the aggressiveness of treatments, alterations in body image, a decrease in the level of functional autonomy or role alterations" (p. 25). Thus, with cancer it is usually difficult to identify an exact, or single, stressor that precipitates PTG. Second, while acute traumatic events are often caused by an external source; cancer has an internal nature and origin. The authors proposed that it is this internal reference point that makes it more difficult for survivors to avoid or ignore signs of the trauma (such as not feeling well physically), and that may also play a key role in changing assumptions about the individual's self.

Third, unlike acute traumas, in which the recurrence of intrusive cognitions is associated with the past traumatic event, with cancer many of the intrusive cognitions are fears related to future health. Cancer survivors, unlike other trauma survivors, must live with the threat of disease recurrence (Carboon, et al., 2005). For instance, in a study with breast cancer survivors conducted by Bower, Meyerowitz, Desmond, Bernaards, Rowland and Ganz (2005), approximately $40 \%$ of the participants reported a persistent 
worry about the possible recurrence of the illness five years after diagnosis. Sumulla, et al. (2009) asserted, "in a cancer-associated trauma what is present is an ill-fated future, resulting in the inability of the patients to picture themselves over time because of the distress this causes" (p. 26).

Fourth, it is not easy to establish the onset and termination of cancer. Typically, with a cancer diagnosis comes the progressive presence of adverse events related to treatment, side effects, and changes in prognosis such that it feels like a "long obstacle race" (Sumulla et al., 2009). Finally, survivors of cancer often experience a certain level of control over treatment and its outcome. In contrast with the uncontrollable nature of an acute trauma, medical knowledge related to the mechanisms involved with cancer may allow survivors some means of changing the way in which the illness develops (e.g.; diet, exercise, etc.). Komura and Hegarty (2006) asserted that many of the positive changes described by cancer survivors are associated with a greater sense of perceived control over their lives and selves, and this perception is a typical characteristic of illness-related trauma.

Experience Of Being A Survivor: Prominent Role of Uncertainty \& Fear

To better understand what it means to be a cancer survivor, Allen, Savadatti and Levy (2009) conducted six focus groups with 47 breast cancer survivors, who had completed treatment in the past 12 months. Participants were asked about the transition from being a "patient" who is actively in treatment, to a "survivor" who has completed treatment. The most prominent theme in these groups centered around positive aspects of the cancer experience. These changes included: enhanced appreciation for life, greater willingness to do things that were once put off, feeling empowered by surviving, and an 
increased ability to "not sweat the small stuff" (p. 74). Yet, the majority of women also expressed constant and distressing fears about disease recurrence. Often these fears were triggered by physical symptoms of unclear origin. Related to fear of recurrence where reports of emotional distress. For some women, it felt as though anguish could arise at any time without a specific trigger.

While the completion of successful cancer treatment may be a cause of celebration for many survivors, the end of treatment was associated with a sense of loss for many women in the focus groups. The loss experienced was related to the cessation of monitoring by, and support from, medical professionals, and a clear "action plan" against cancer. The women also reported decreased contact with other cancer survivors. Finally, the women talked about difficulty returning to their pre-cancer life including: the sense that life would never feel "normal," the struggle to resume responsibilities, the burden of needing to "be strong" for family and friends, and uncertainty regarding the future. The authors also noted that for younger women, the unknown impact of treatment on fertility was typically a prime concern (Allen et al., 2009).

Distress and physical symptoms related to cancer can continue for many years after diagnosis and treatment. While there is a general understanding of, and sympathy for illness in our society, it has been argued that the challenges of survival are less well recognized (Little, Paul, Jordens \& Sayers, 2002). One proposed reason why cancer leaves consequences that persist well beyond diagnosis and treatment is because it is an "extreme experience...that leaves no aspect of identity untouched" (Little, et al., 2002, p. 176). The authors explained,

The physical, embodied component of identity has been challenged, and its vulnerability made clear. There may also be physical changes in the body as a result of 
the extreme experiences, such as a colostomy or a surgical scar. The content of cognition, the perceptions and remembered experiences that make up the background to our thinking, has been changed. There is now available to it the knowledge that comes from the extreme experience. (Little, et al., 2002, p. 176-177)

A paradoxical rise in anxiety has been found at the end of treatment as the survivor now must contend with an uncertain future without frequent contact with medical services (Brennan, 2001; Kaiser, 2008). Kaiser (20008) performed a qualitative study with 39 women who had completed treatment (surgery, chemotherapy, or radiation) for breast cancer 3-18 months prior to the interviews. One survivor talked about the contrast between the precise, medical periods of diagnosis and treatment, and the vague, uncertain period following treatment.

There was so much definition to the cancer, you know the cancer is this grade, and it's estrogen receptor positive, and you're this age and all these factors and this is what we can do. Everything was so precise and now it's like you're back to the unknown again like I was before, and I don't like that. (Kaiser, 2008, p. 84)

A paper published in the Journal of Aging Studies in 2005 presented the experiences of breast cancer survivors living years beyond diagnosis. The dialogue presented in the paper was taken from focus group transcripts that were conducted to help create a theatrical production in Ontario, Canada about life after breast cancer. The focus groups were comprised of 10 cancer survivors (nine breast, one cervical) who were at least four years disease free (Sinding \& Gray, 2005). The women spoke of ongoing pain, scars, or perhaps having only one breast from surgery, lingering anxiety and depression, fatigue and low energy as well as the loss of predictability of energy. The awareness that cancer might recur was central to the experience of survivorship. The potential for metastatic disease carried with it a change in the meaning of bodily symptoms. The 
authors explained, "Certain signs, once neutral, became charged; aches or pains that would have been treated casually are now laced with danger" (p. 153).

The women discussed how they know they may live for many years, even perhaps the rest of their lives, without ever receiving another cancer diagnosis; yet, they described "living under threat," of anticipating, at some level, cancer returning. This experience of living under threat was perceived to separate the survivors from their peers who had never experienced cancer. One survivor pointed out that others, based on the universality of death, often minimize the sense of ongoing threat: 'any of us could be hit by a bus!' Yet, as another survivor noted, "Most people don't go around worrying they are going to be hit by a bus and most people do not try to organize their lives to avoid buses" (Sinding \& Gray, 2005, p. 154).

\section{Cancer and Posttraumatic Growth}

Traumatic events, including cancer and other life-threatening illnesses, can undermine a person's ability to cope, create symptoms of depression and anxiety, and decrease psychological well-being. The psychological toll of illness is related to the challenge and threat the illness presents to one's core beliefs and assumptions about themselves and the world (Janoff-Bulman, 1992). Lepore and Helgeson (1998) explained, "The life-threatening and unpredictable nature of cancer, as well as the changes it can impose on daily life, can cause its victims to question core beliefs they hold about themselves, their relationships with others, and their future" (p. 90). For example, the authors described that prostate cancer can challenge a man's assumptions about being invulnerable to illness, having an active and fulfilling retirement, providing for his family, and satisfying his and his partner's sexual desires. Moreover, prostate cancer is 
likely to especially challenge a man's masculine identity, because it can cause fatigue and lessen control over important bodily functions, including sexual function and continence (Lepore \& Helgeson, 1998).

For many people the threat of cancer is believed to have been the prompting force for restructuring their lives in more meaningful ways, thereby creating the perception that cancer was beneficial. When individuals find some positive meaning in their illness, their ability to cope is enhanced, they might feel better about themselves, and they suffer less (Affleck \& Tennen, 1996; Taylor, 1983). According to Taylor (1983), finding meaning in adversity is self-empowering. It is a cognitive adaptation that lessens the negative impact of traumatic events and helps the individual master future challenges.

Taylor (1983) reviewed data from interviews with 78 women with breast cancer, and found that when the women were asked if the changes they reported in their lives since cancer were positive or negative, only $17 \%$ reported any negative changes in their lives. Fifty-three percent reported only positive changes, and the remainder reported no changes. Moreover, participants tended to see themselves as presently better adjusted than before diagnosis. Taylor (1983, p. 1165) concluded that the women possessed a "remarkable ability to construe personal benefit from potential tragedy."

A study performed over a three-year period with 56 adult bone marrow transplant (BMT) cancer survivors found that most survivors found benefit from cancer (Tallman, Altmaier \& Garcia, 2007). More specifically, at one-year post BMT, 91\% of participants indicated at least one benefit from their experience; most frequently in the "life perspective domain." At three years post BMT, again, 91\% of participants found benefits from their experience. The majority of participants reported benefits in life perspective, 
followed by perception of self, interpersonal relationships, and spiritual or religious benefits. Moreover, it was found that individuals who found more benefit at year one were less depressed and had better physical functioning at year three than were participants who found less benefit at year one (Tallman et al., 2007).

Key Variables Associated with Posttraumatic Growth Following Cancer

\section{Demographic Variables}

Numerous studies have demonstrated that demographic variables, such as ethnicity, marital status, gender, religious affiliation, and age are unrelated, or inconsistently related, to individual differences in PTG among individuals with cancer (Bellizzi \& Blank, 2006; Lechner et al., 2003; Stanton et al., 2006; Tomich, \& Helgeson, 2004; Widows, Jacobsen, Booth-Jones, \& Fields, 2005). However, given the inconsistent relationship between some of these variables and PTG, along with the fact that the majority of studies that have examined PTG in cancer have been conducted with breast cancer survivors, certain demographic variables, such as age and gender, require further investigation.

Age

Prior research examining the impact of age on PTG has revealed non-significant results, although several studies have demonstrated significant negative associations, such that younger adults with cancer report greater PTG than do older adults (Stanton et al., 2006). For example, Manne, Ostroff, Winkel, Goldstein, Fox, and Grana (2004) found that younger breast cancer survivors had higher PTGI scores shortly after surgery, as well as nine and 18 months later compared to older breast cancer survivors. The researchers suggested that the younger survivors in the study might be more aware of and 
motivated to conform to expectations to adopt a positive attitude when dealing with cancer than older adults. It has also been hypothesized that one reason differences emerge in PTG between young and older survivors is because onset of disease is a more normative experience for older adults, whereas a cancer diagnosis requires more developmental readjustment for younger adults and is therefore more distressing for them (Stanton et al., 2006). Bellizzi (2004) reported, “Those diagnosed in their thirties and forties likely face a different set of life challenges, expectations, and demands when compared to individuals diagnosed in their eighties" (p. 269). Furthermore, the researcher hypothesized that younger cancer survivors may experience greater PTG than older survivors because they realize they have more time left in their lives to accomplish chosen goals.

Using a sample of 74 cancer survivors ranging in age from 23 to 93 years, Bellizzi (2004) examined three age groups: 26-41 years, $42-54$ years, and 55 years and over in relation to perceptions of PTG as measured by the PTGI. It was found that both younger adults as well as midlife adults reported significantly higher scores of PTG than older adults. When examining the subscales of the PTGI, it was revealed that younger and midlife cancer survivors reported significantly more growth in 'new possibilities' than older survivors. There were no significant differences between younger and midlife survivors. This same pattern was found for 'personal strength' and 'appreciation of life'. With regard to 'spiritual change' midlife survivors reported more growth than older survivors, and again there were no differences between younger and midlife survivors. Bellizzi (2004) speculated that lower scores of PTG may be found among older individuals because of where they are in the life cycle. Perhaps with age comes a 
"peaceful acceptance" about life, and growth may simply not be as important to older survivors.

Gender

Gender may influence how one copes with and processes his/her cancer experience, which in turn, influences the emergence of growth. However, research on the relationship between gender and PTG is mixed. Some studies propose that females report both more distress and more growth compared to men, while other studies report no differences (Salsman, Brechting, Segerstrom, Carlson \& Andrykowski, 2009). In a study conducted with 55 colorectal cancer survivors, of which $58.9 \%$ were female, it was found that gender was unrelated to psychological variables including: cancer-related intrusive thoughts, PTSD, and PTG (Salsman, et al., 2009). Lepore and Helgeson (1998) argued that talking with empathic and supportive others facilitates cognitive processing and growth. Yet, the researchers asserted that prostate cancer, in particular, may be difficult to talk about with close others because it is related to a man's intimate anatomy and creates problems in bodily functions that ordinarily might not be discussed.

In a qualitative study with 34 prostate cancer survivors and their partners (Gray, Fitch, Phillips, Labrecque \& Fergus, 2000), which examined issues related to coping and support, it was found that many of the men minimized the impact of their cancer and either seemed to experience less distress overall when compared to their partners or went to greater lengths to avoid acknowledging their distress. The researchers explained that, in general,

Ill men are in a psychological bind. They may feel vulnerable, and may experience more intense emotions than they are accustomed to feeling. Consequently, they may also feel a greater need for emotional support. But these experiences run counter to their identities as men. To express their distress or to actively seek support has the 
potential for undermining their sense of self. Minimization of impact thus becomes understandable. (Gray et al., 2000, p. 545)

The researchers also found that the survivors flipped back and forth from talking with their partners and withdrawing. The researchers explained that sometimes the men "go with the needs the illness evokes" and at times "fight those needs in favor of meeting their expectations for themselves" (p. 545). Overall, the impact of the disease on the survivors was often hidden as they struggled to stay in control of their emotions and lives (Gray, et al., 2000). If men are more likely to downplay their cancer experience and not acknowledge their distress due to gender expectations, it seems reasonable that they would experience less growth compared to females as the emergence of growth requires an acknowledgment of distress and cognitive processing, which for many is facilitated by talking with others.

To examine the amount of growth reported by prostate cancer survivors, Thornton and Perez (2006) conducted a study with 82 prostate cancer survivors, which assessed PTG one year after the men underwent a radical prostatectomy. The researchers found that none of the demographic and medical information collected, including employment status, type of surgery (standard versus nerve-sparing), and disease stage, were related to PTG. In general, the survivors reported a modest degree of PTG one year after treatment. Coping by using positive reframing $(p<0.001)$ and emotional support $(p=0.01)$ was significantly related to PTG. Levels of PTG were slightly lower in the sample of prostate cancer survivors compared to samples of breast cancer survivors. The researchers hypothesized that lower scores for prostate survivors may have been related to the fact that stress symptoms were lower in the sample of men one year after treatment compared to stress symptoms of breast cancer survivors that were assessed as much as two years 
post-diagnosis or treatment. In addition, echoing the conclusion above, the researchers postulated that gender socialization differences between men and women could have impacted reports of PTG (Thornton \& Perez, 2006).

It has also been proposed that both men and women seek emotional support and information following cancer, but that they express themselves differently. A 2007 study conducted by Gooden and Winefield investigated two online discussion boards for breast and prostate cancer survivors in order to examine the expressive content of the survivors' postings. Using a qualitative research approach it was found that the two primary reasons survivors used both discussion boards were information sharing and emotional support, and both men and women used the boards for these reasons equally. However, there were differences in how the survivors communicated (Gooden \& Winefield, 2007). Men provided more accounts from research and medical reports than women and spent more time discussing disease-site specific concerns, such as sexual dysfunction. Moreover, men used humor as a way to cope whereas women used humor less. Regarding sharing distress, women clearly expressed emotions, whereas men tended to imply their emotions. When offering encouragement to other survivors, women tended to provide nurturance and affection, whereas men emphasized strength and used "battle like terminology, as though they were at war with cancer" (Gooden \& Winefield, 2007, p. 111). Results of this study highlight the differences in how men and women tend to express themselves. It is important to note that growth measures may be more in accordance with how women are socialized to express their thoughts and feelings, which may be part of the reason why many studies find women reporting more growth than men. 
Zwahlen, Hagenbuch, Carley, Jenewein and Buchi (2010) reiterated the above conclusion after conducting a study examining the effects of gender and role on benefit finding in couples. The sample of 224 cancer survivors (123 men and 94 women) and their partners completed questionnaires, including the PTGI. The participants had several types of cancer including, among others: skin, intestinal, breast, lung, and leukemia. The results revealed gender did contribute to variation in PTGI scores such that PTGI total scores for all women in the sample were greater than those reported by the men. The researchers suggested that females may be more willing or able to express personal growth experiences or the PTGI itself may not capture the "engagement in emotional work in men" (p. 17).

It is worth noting that an alternative hypothesis for why prostate cancer survivors, in particular, are less likely to report PTG than breast cancer survivors is that, based upon trends in five-year survival rates, more individuals survive five years with prostate cancer (99\%) compared to breast cancer (89\%, National Cancer Institute, 2007). Because fewer people die from prostate cancer than breast cancer, it may be perceived as less threatening, and thus, less growth is reported. However, survival rates based off of group data do not speak to the individual differences among survivors, and perceived threat may be related to many variables, such as type of treatment received, treatment side effects and degree that beliefs are challenged. Furthermore, as will be discussed below, a survivor may subjectively feel a great degree of threat regardless of objective data, such as medical information and relevant statistics. Upon hearing a diagnosis of prostate cancer, a man may be very worried that he will die, regardless of the fact that, in general, fewer individuals die of prostate cancer compared to other types of cancer. 


\section{Time}

Intuitively, it seems that a positive correlation would be expected between PTG and time, as more time to process the meaning and impact of cancer might be associated with the discovery of more benefit and positive life changes. For example, measures of PTG taken soon after a traumatic event might reflect a cognitive strategy that individuals use to reduce distress, rather than reflecting actual change or growth (Helgeson, Reynolds, \& Tomich, 2006). As Helgeson, et al. (2006) stated, "It is difficult to imagine that true growth can occur within days of a traumatic event" (p. 811). Butler (2007) explained that growth is more likely to be reported in hindsight when an individual reflects upon his/her experience because he/she has used coping methods and other efforts over time to process what has occurred. A longitudinal study conducted by Manne et al. (2004) found a consistent and significant increase in PTGI scores over 18 months among women with breast cancer. In general, a meta-analysis revealed that benefit finding is more likely to be related to a good outcome when a longer time has elapsed since the trauma (Helgeson, et al., 2006).

It is likely that when an individual is initially diagnosed with cancer, he/she experiences a high level of distress and feelings of being overwhelmed, as the individual is confronted with a large amount of new information that needs to be processed. For instance, results from a longitudinal study conducted with women with various types of cancer indicated that the cancer diagnostic period is one of acute stress (Andersen, Anderson, \& deProsse, 1989). Carboon, et al. (2005) asserted that during treatment survivors report being "intently focused on the goal of 'getting better."' Thus, all their resources are directed to managing the practical aspects of treatment. It is only when 
treatment is complete that many survivors find time and energy to process and reflect on their experience. Therefore, it typically takes time for PTG to emerge. Sears, Stanton and Danoff-Burg (2003) found that among a sample of women with early-stage breast cancer who had completed medical treatment, greater perceived cancer stress and longer duration since diagnosis at study entry were related to PTG one year later. Findings were consistent with the notion that more intensive initial engagement with a stressor (as indicated by high thought intrusion, avoidance, and stress appraisals), coupled with more time to process the stressor, facilitates PTG.

Some research, conducted with time periods ranging from two weeks to eight years after a traumatic event, has suggested that PTG is unrelated to time since the trauma occurred, and that there may even be a negative relationship between time and growth (Linley \& Joseph, 2004; Tedeschi \& Calhoun, 1996). Stanton et al. (2006) reported that in two studies that found a negative relationship between the variables, the participants were on average more than three years post-diagnosis of their cancer, as compared with a diagnosis duration of less than two years in three of the four studies that found a positive relationship. Therefore, it has been suggested that the "relationship between PTG and time since diagnosis may be stronger in the one to two years following diagnosis and treatment than after several years of survivorship" (Stanton et al., 2006, p. 159).

Overall, the literature on the relationship between time and PTG is inconsistent, which is likely related to methodological issues in the research. For instance, many studies use participants who are newly diagnosed and likely still undergoing primary treatment (e.g., surgery, radiation, chemotherapy) or those who are no more than a few years post-diagnosis (Stanton et al., 2006). There is less information available regarding 
longer-term survivors. Further, studies generally utilize a cross-sectional design. Because there is a lack of longitudinal data (Helgeson et al., 2006; Stanton, et al., 2006), researchers are uncertain of the emergence and role of growth over time.

Nonetheless, it has been suggested that it is not time per se that facilitates PTG, but rather intervening events and processes (Linley \& Joseph, 2004). Considering the cognitive processing model of trauma, it is probable that the longer the time since being diagnosed with cancer, the more opportunity for deliberate cognitive processing (versus the automatic and intrusive rumination that typically occurs in the immediate aftermath of trauma), which would increase the facilitation of PTG (Cordova et al., 2001; Helgeson et al., 2006; Sears et al., 2003).

\section{Types of Cognitive Processing}

By and large, little research has been conducted that examines the relationship between specific types of cognitive processing and growth. Manne et al. (2004) conducted a study with 162 women with breast cancer and their partners, and found that women who engaged in more contemplation about the potential reasons why they might have developed breast cancer experienced more growth over time. It was also found that engaging in more attempts to search for meaning in breast cancer was marginally associated with gains in growth. However, other cognitive processes, including intrusive thoughts, searching for a cause for developing cancer, and positive reappraisal were not associated with growth. The researchers concluded that not all cognitive processes facilitate growth. For instance, they proposed that searching for a cause of one's cancer may lead to self-blame, which has been found to be associated with poorer adaptation to breast cancer (Manne et al., 2004). 
Another study examined whether cognitive processing was associated with PTG after stroke in a sample of 60 stroke survivors (Gangstad, Norman \& Barton, 2009). The researchers found statistically significant $(p<.01)$ positive correlations between scores on the Posttraumatic Growth Inventory and four of the five Cognitive Processing of Trauma Scale subscales: Positive Cognitive Restructuring, Downward Comparison, Resolution, and Denial ( $r=.52, .29, .44$ and .38 , respectively). Thus, higher levels of growth were associated with these four subscales. It was concluded that cognitive processing of the traumatic event is an important process for stimulating PTG. Interestingly, Denial is one of two subscales (Regret is the other) that are thought to represent minimal and/or negative cognitive processing and thus, theoretically, one would assume that denial would be negatively, not positively, related to growth. Yet, Bonanno (2004) has written on the potentially adaptive role of various kinds of cognitive processing, such as denial, self-enhancement and dissociation from stressful events. Therefore, it is possible that in some instances denial is helpful and in others it is not. There is a gap in the current literature related to understanding how different types of cognitive processing influence growth.

Finally, 83 people with newly acquired limb loss participated in a study which investigated symptoms of depression, PTSD and PTG (measured with the PTGI) six and 12 months following amputation (Phelps et al., 2008). The researchers examined cognitive processing as a potential predictor of the psychological outcomes. Positive Cognitive Processing consisted of benefit-finding, accommodation of belief structures, and resolution. Negative Cognitive Processing was defined as ruminative thoughts, anger, sense of victimization, and blaming. It was found that Positive Cognitive Processing was 
significantly related to depressive symptom severity at six and 12 months $(r=-.27$ and .33 , respectively) and to the level of PTG at 12 months $(r=.33)$, such that greater levels of positive processing were associated with lower symptom severity and more growth. Negative Cognitive Processing was significantly related to depressive and PTSDsymptom severity at six months $(r=.29$ and .36 , respectively), such that greater levels of negative processing were associated with greater levels of distress. Moreover, cognitive processing was found to account for 15 percent of the unique variance in PTG at 12 months, with higher levels of positive processing associated with higher levels of growth (Phelps et al., 2008).

The above researchers also found that demographic factors were unrelated to PTG, which is consistent with prior research (Linley \& Joseph, 2004). Impressively, in their study, positive cognitive processing within nine weeks of amputation predicted growth at 12 months. Interestingly, negative cognitive processing was found to be basically unrelated to PTG at any time. It was concluded that the findings support a cognitive-processing model of PTG in which deliberate thought characterized by meaning-making, as opposed to nonproductive rumination, facilitates PTG (Phelps et al., 2008).

\section{Perceived Threat: Overview}

Threat of mortality as well as threat to a person's overall belief system are thought to be a stimulus for PTG in cancer survivors, as a diagnosis of cancer involves an inherent threat to mortality and often challenges how one sees him/herself, others and the world at large. Thus, a general theme in the literature on growth following cancer is that 
the greater the impact of the cancer experience (i.e., larger threat to physical well-being and beliefs/assumptions), the greater the potential for PTG (Stanton et al., 2006).

\section{Curvilinear Relationship Between Threat and Growth}

Based upon Tedeschi and Calhoun's (2004) model of PTG, if an event is not serious enough to provoke a re-examination of one's fundamental beliefs, schemas are unlikely to be shattered. Therefore, searching for meaning in the cancer experience might not take place, and the individual would not believe that much had changed in his/her life as a result of cancer. Conversely, the experience of very advanced disease might prompt such a high life threat that an individual cognitively shuts down any search for meaning and benefit (Lechner et al., 2003). Carboon, et al. (2005) explained that cognitive processing, and subsequent perception of PTG, might be contingent on the ending of immediate threat upon successful treatment, thereby arguably allowing a survivor to have enough emotional and physical energy to devote to processing his/her cancer experience.

Evidence exists that a curvilinear, rather than a linear, relationship exists between degree of trauma exposure, PTG and trauma-related distress (Butler, Blasey, Garlan, McCaslin, Azarow, Chen, et al., 2005; Kleim \& Ehlers, 2009; Lechner et al., 2003; McCaslin, Zoysa, Butler, Hart, Marmar, Metzler, et al., 2009). For example, using a large convenience based Internet sample participating in a longitudinal study following the terrorist attacks of September 11, 2001, Butler, et al. (2005) found a curvilinear relationship between PTG and PTSD symptoms, with the highest level of growth reported by those who endorsed a moderate level of PTSD symptoms. Further, results indicated that initial PTG (nine weeks post 9/11) was related to higher levels of trauma symptoms (measured by the PTSD Checklist-Specific), positive changes in worldview 
(measured with the Changes in Outlook Questionnaire), more denial, and less behavioral disengagement (measured with the Brief COPE). Levels of PTG also declined somewhat over time with the exception of 'spiritual change.' PTG levels at follow-up assessment (mean 6.5 months post-attacks) were primarily predicted by initial PTG levels; however, decreases from initial trauma symptoms and increases in positive worldview, acceptance, and positive reframing were also associated with higher reported PTG at follow-up. The authors concluded that the results "suggest that there may be a range of traumatic experience most conducive to growth and they also highlight the important contributions of cognitive and coping variables to psychological thriving in short-and longer-term periods following traumatic experience" (Butler, et al., 2005, p. 247).

Given the evidence of a curvilinear relationship between distress and growth, McCaslin, et al. (2009) argued that a sample with a small range of PTSD symptoms could conceivably yield a positive, negative or no relationship to growth depending upon the level and range of symptoms, and segment of the curve sampled. The researchers also explained that the curvilinear relationships found between PTG and PTSD suggest that an optimal level of immediate and subsequent distress may facilitate PTG, whereas low levels of distress may be insufficient to foster growth, and an overwhelming amount of distress, at the time of an event and following it, may impede the development of PTG.

\section{Threat to Physical Well-Being}

The threat of mortality that comes with a cancer diagnosis is consistent with existential theory (Frankl, 1959), and more specifically, the concerns that arise when one is confronted with the finite nature of his/her life. Weisman and Worden (1976) described the "existential plight in cancer" as a period of time that begins with initial and definite 
diagnosis and continues for two to three months. The researchers interviewed and assessed 120 newly diagnosed cancer survivors using semi-structured interviews and assessments, specifically the Minnesota Multiphasic Personality Inventory (MMPI), the Thematic Apperception Test (TAT), and scales that were designed for the study (a modification of the General Coping Strategies [COPE], Resolution [RES], Inventory of Predominant Concerns [IPC] and the Index of Vulnerability [VUL]). The survivors were assessed at about 10 days after diagnosis, then at four and six-week intervals until three to four months had elapsed. Overall, the authors found that the primary signs of this period were predominance of life/death concerns as the individual's very existence seemed endangered. Weisman and Worden (1976) suggested that cancer survivors, regardless of the prognosis or site, are primarily concerned about dying. Other concerns about health, family, finances, work, and so forth are largely secondary during the first few months after initial diagnosis.

Being confronted with one's mortality may elicit a re-evaluation of life goals and priorities, such that individuals have a greater appreciation of life, interpersonal relationships, spirituality, and personal strengths and skills (Cordova, et al. 2001; Fromm, Andrykowski \& Hunt, 1996; McMillen, Smith \& Fisher, 1997; Wortman, 2004). For instance, a study conducted with 90 male cancer survivors revealed that a majority of the participants reported an increased ability to appreciate life, and an enhancement in their interest and concern for significant others. The authors concluded that the cancer experience had a "humanizing impact" on the survivors (Cella \& Tross, 1987).

It has been suggested that the greater the life threat posed by the stressor, the greater the opportunity for growth (Cordova, Cunningham, Carlson, \& Andrykowski, 
2001); however, there have been few studies that have assessed perceived threat of cancer. Most studies in the psycho-oncology literature have utilized objective stage of disease as a measurement of threat. For instance, a study that examined benefit-finding and quality of life in 364 women with Stage I, II, and III breast cancer found that stage of disease was associated with benefit-finding (Tomich \& Helgeson, 2004). Women diagnosed at Stage II perceived more benefits as a result of their experience than did those diagnosed at Stage I. Furthermore, the mean on the benefit-finding measure for women diagnosed at Stage III was higher than those diagnosed at Stage I and II, but this difference was not statistically significant, likely because there were only 22 women with Stage III cancer. The authors concluded that women diagnosed with more severe disease perceived more benefits following diagnosis than did women diagnosed with less severe disease and that a potential reason for this difference is because women with more advanced disease may have more critically examined their experience (Tomich \& Helgeson, 2004).

These results, and the conclusion drawn from them, need to be examined critically as research has shown that threat measured using stage of disease is often not a valid indicator of the subjective level of threat a survivor may be experiencing (Cordova, Giese-Davis, Golant, Kronenwetter, Chang, \& Spiegel, 2007; Lechner, Zakowski, Antoni, Greenhawt, Block, \& Block, 2003). For example, if degree of cognitive engagement and reflection is based upon amount of subjective threat, which is not dependent on disease stage, then someone with Stage I cancer may feel just as much, or possibly more, threat than someone with Stage III cancer. In that case, the person at Stage I might deliberately think about and process his/her experience. 
Utilizing a more subjective measure of threat, Cordova et al. (2001) conducted a study, which investigated predictors of PTG among 70 women with breast cancer. The researchers examined perceived threat based upon Diagnostic and Statistical Manual of Mental Disorders-IV criteria for PTSD. They asked participants two questions: "Did you perceive being diagnosed with and treated for breast cancer as a threat of death or serious injury or a threat to your physical integrity?" and "Given your experience with breast cancer, has your response ever involved intense fear or helplessness?" The coefficient alpha for this two-item alternative measure of the stressor criteria was .68 (Cordova et al., 2001).

It was found that $61 \%$ of breast cancer participants endorsed both of the questions above; $80 \%$ perceived breast cancer as a threat to life or physical integrity; and $64 \%$ responded with fear or helplessness. Moreover, the total score on the Posttraumatic Growth Inventory was significantly correlated with scores on the two-item stress criteria measure $(\mathrm{r}=.38, p<.001)$, such that greater PTG was associated with breast cancer being perceived as a traumatic stressor. The researchers concluded that for stressors to elicit positive change, they must have been threatening enough to challenge one's assumptions and to elicit coping responses (Cordova et al., 2001).

A further study conducted by Cordova et al. (2007), with 65 breast cancer survivors, utilized the same criteria as the above study to measure "Stressfulness of the Cancer Experience," and found that approximately $66 \%$ of the sample reported that they perceived their cancer experience as a threat to their life or physical well-being, and 59\% indicated that their response involved fear or helplessness. It was found that perception of cancer as a traumatic stressor was a unique predictor of PTG $(p<.05)$, such that those 
who perceived cancer as traumatic reported greater PTG. Results also revealed that objective indices of cancer severity, such as stage of disease or treatment received, were not related to PTG. The researchers suggested that in line with stress and coping theories, perceived, rather than objective threat posed by a stressor appears to be a better predictor of adjustment (Cordova et al., 2007).

Salsman, et al. (2009) conducted a study with 55 colorectal cancer survivors which examined emotional coping as a predictor of PTG. Participants were all posttreatment and within six to 18 months post-diagnosis. Perceived threat was measured using DSM-IV stressor criteria. Results indicated that few participants (16.4\%) perceived their cancer experience as a traumatic stressor; however, perceiving the cancer experience as traumatic was associated with reports of appreciation of life and greater personal strength over time. The researchers concluded PTG might continue to increase posttreatment as patients begin to live life as a cancer survivor (Salsman et al., 2009).

Bellizzi and Blank (2006) asked 224 breast cancer survivors to use their recollection to rate the emotional impact they experienced in relating to others, purpose in life, and appreciation of life at the time of diagnosis. Results indicated that the perceived emotional impact of the disease on specific domains of life was significantly related to perceptions of PTG. More specifically, women who rated a high emotional impact at the time of diagnosis reported higher levels of PTG in their relationships with others, purpose in life and appreciation for life compared to women who did not (Bellizzi \& Blank, 2006).

Lechner et al. (2003) investigated the relationships between benefit-finding, sociodemographic and disease-related variables in a sample of 83 men and women with 
various types and stages of cancer. The researchers measured perceived threat by asking the participants to provide a percentage rating of how likely they felt it was that they would die of cancer; the rating was made on a scale of zero to 100 percent. Lending support to the notion that perceived threat is an important determinant of PTG, the researchers found that after statistically controlling for stage of disease, perceived threat was significantly correlated with benefit-finding $(r=0.24, p<0.05)$.

This finding, along with the results of the above studies, indicates that irrespective of objective life threat (i.e., stage of disease), perceptions of threat appear to influence the facilitation of PTG. Lechner et al. (2003) concluded that different levels of threat or prognostic uncertainty should produce different responses to cancer. Bower, Meyerowitz, Desmond, Bernaards, Rowland and Ganz (2005) reported that there is evidence that greater impact of cancer is associated with perceptions of both vulnerability and positive meaning. For instance, studies have shown that younger survivors, as well as those who perceive a greater physical threat associated with cancer and who undergo more risky medical treatment, report more positive changes (Cordova, et al., 2001; Lechner, et al., 2003).

The majority of studies on PTG and cancer have examined individuals diagnosed with breast cancer. A study by Widows et al. (2005) was conducted with 72 bone marrow transplant patients with the aim of extending previous research to a population of cancer survivors undergoing more aggressive treatment and who are at a greater risk for mortality than are women with non-metastatic breast cancer. Contrary to hypotheses, PTG was found to be unrelated to risk of disease recurrence or progression $(p<.19)$, a variable that might serve as an objective measure of degree of life threat. 
Overall, the above study found that reports of PTG were common among participants, yet not more so than reports of PTG in other populations of cancer survivors. More specifically related to threat, PTG was not significantly related to the total stress appraisal score $(p=.09)$; however, it was significantly positively associated with postBMT recollections of greater emotional distress $(p=.01)$ and greater concern for life $(p=$ .02) during BMT. Thus, in general, similar to other research findings, the researchers explained that survivors' subjective concerns about mortality were associated with PTG, whereas an objective measure of mortality risk was not (Widows et al., 2005).

Summarizing the research, Brennan (2001) explained that since cancer diagnosis poses a future threat that is likely to be influenced by the individual's unique belief system, typically the individual's perception of their prognosis may be a more accurate measure of the traumatic impact of disease threat, rather than the more objective prognosis made by medical professionals based on clinical staging.

\section{Threat to Assumptive World}

As mentioned above, a traumatic event has the potential to greatly challenge an individual's beliefs about him/herself, others, and the world. It has been proposed that in order to "make meaning" of the event, individuals must either change their view of the trauma to make it fit into their existing worldviews, or change their worldviews to accommodate the trauma. Park and Folkman (1997) asserted that individuals alter either their global beliefs and goals, or their appraised situational meaning of the traumatic event in order to reduce their distress. While this study conceptualizes PTG as an outcome rather than a coping strategy, Tennen and Affleck (1998) suggested that 
perceiving positive changes or personal growth following trauma is an adaptive strategy for reducing the upsetting nature of the event.

Cognitive processing is considered crucial to resolve discrepancies between an individual's previously held beliefs and current situation that they appraise as violating those beliefs (Park \& Blumberg, 2002). Thus, a traumatic event must sufficiently challenge an individual's beliefs in order to set in motion cognitive processing that potentially may lead to PTG. Conversely, if the event does not disrupt an individual's beliefs, his/her distress is likely to be less severe, and subsequently there is less of a psychological need to alter his/her worldview to accommodate trauma.

Few, if any, studies have examined the extent to which having cancer challenges an individual's worldview. In a qualitative study conducted by Collins, Taylor and Skokan (1990) 55 female and male cancer survivors were asked about changes experienced in self-views, views of the world, future plans, relationships, and activities/priorities following diagnosis. On average, participants had received a diagnosis of cancer, or had a recurrence, three years prior to the interview. Results revealed that the majority of participants reported changes in their perceptions following diagnosis. Eighty-four percent reported changes in their views of themselves, $83 \%$ in their relations with others, $79 \%$ in priorities/daily activities, $67 \%$ in their plans for the future, and $66 \%$ in their views of the world. Changes in activities/priorities and relationships were primarily positive, whereas changes in views of the self, world, and the future were mixed. More specifically, there was a belief by some participants that the future was now uncertain or threatening. Although respondents reported increased perceptions of threat, 
some of them considered this a positive change as they now had an increased awareness of the uncontrollability of many events (Collins et al., 1990).

As stated previously, Bellizzi (2004) examined PTG in a mixed age and gender sample of cancer survivors. Participants were asked to rate on a 4-point Likert scale "the extent to which cancer impacted their perspective on life" (p. 276) and to explain their answers. The narrative explanation was coded and examined for themes that were associated with the four response choices. The researcher found that younger survivors' perspectives on life were impacted significantly more than older adults, but there were no significant differences between midlife survivors and the other two groups. Graphing the results, it was also found that there was a linear decline in impact for middle and older survivors compared to the younger group. Furthermore, $12 \%$ of the oldest group reported, "having cancer had no impact on their perspective on life" (p. 278).

Of those who reported cancer had impacted their perspective on life "a lot," $64 \%$ described an "increased awareness of the fragility of life and the value of loved ones, and had learned not to worry about the little annoyances in life" (p. 278). The researcher concluded that for this group of survivors, facing mortality and realizing the fragility of life might promote a positive shift in one's beliefs regarding life. For those who reported the impact on life perspective as "somewhat," $68 \%$ reported themes related to mortality. For participants who reported "a little" impact, no consistent themes emerged. However, one 53 year-old prostate cancer survivor reported, "I spend little time reflecting on a situation that I can't control and their outcomes" (p. 281). Finally, for those who stated "no impact" on their life perspective, 58\% reported having other problems that took precedence over cancer (Bellizzi, 2004). 
While the above studies offered useful information regarding changes in beliefs, a missing component in the literature is the degree to which an individual's beliefs are challenged. As noted previously, according to the model of PTG utilized in this study (Tedeschi \& Calhoun, 2004), the more the event challenges an individual's beliefs, and causes them to reflect on various life issues, such as mortality, the greater the potential for PTG. Thus, it is critical that the degree of threat to an individual's belief system, not necessarily changes in the belief system, is measured. Bellizzi (2004) echoed this idea by stating, "It may be important for posttraumatic growth models to examine potential levels of "seismic" events. If different intensity levels exist, do they correspond to different levels of reported posttraumatic growth?"(p. 282).

Anxiety \& Depression in Cancer Survivors

As noted previously, diagnosis of cancer often generates fear and unrest in the lives of survivors. Cancer has the potential to disrupt all aspects of daily life, and often creates anxiety, anger, sadness, and depression as patients struggle to process and work through the many decisions that confront them. It has been proposed that "somatic distress," defined as preoccupation with physical symptoms and fear of recurrence, is almost universal and part of a "normal" response to cancer (Somerfield, Stefanek, Smith, \& Padberg, 1999). A longitudinal study conducted with women who had a variety of cancer diagnoses revealed that the emotional distress experienced by survivors appeared to be primarily defined by depressed and confused moods, with anxiety being the second most frequent source of distress (Andersen et al., 1989).

Prevalence rates for anxiety and depression disorders amongst cancer survivors are generally in the range of 10 to $30 \%$, with rates of various anxiety disorders equivalent 
to, or greater than, those of depression in many cases (Roy-Byrne, Davidson, Kessler, Asmundson, Goodwin, Kubzansky et al., 2008). A particular factor that has been shown to increase the risk of co-morbid depression and anxiety is severity of cancer, as manifested by significant pain, declining performance status, or the need for ongoing treatment (Massie, 2004).

Results from a 2002 to 2006 National Health Interview Survey, using a sample of 4,636 survivors of adult-onset cancer of five years or more, and 122,220 respondents who were never diagnosed with cancer, revealed that survivors were more likely to experience "serious psychological distress" (SPD) than non-survivors. Moreover, survivors remained significantly more likely to experience SPD after adjustment for sociodemographic and clinical characteristics (Hoffman, McCarthy, Recklitis, \& Ng, 2009). SPD was measured by the $\mathrm{K} 6$ scale, which is a validated reliable screening scale that is designed to assess nonspecific psychological distress that is severe enough to cause moderate to severe impairment in social, occupational or school functioning and to require treatment. An important methodological strength of this study is that it was designed to produce a nationally representative sample that reflects ethnic and socioeconomic diversity.

The researchers proposed several reasons why a cancer history can affect current psychological health including: a) cancer diagnosis and treatment can cause delayed negative effects on physical health and functioning, negative affects on social adaptation, employment opportunities or insurance coverage; b) an underlying sense of loss for what might have been in life; c) or an underlying fear of recurrence and death. Overall, it was found that "approximately 1 in 18 non-institutionalized long-term survivors of adult- 
onset cancer who reside in the United States report SPD based on a general screening instrument" (Hoffman, et al., 2009, p. 1279).

A study conducted by Zabora, Brintzenhofeszoc, Curbow, Hooker, and Piantadosi (2001) found that in a sample of over 4000 cancer survivors, the overall prevalence rate of distress was $35.1 \%$. Breast cancer is arguably the most studied cancer in terms of the psychological and psychosocial aspects of the disease. It has been suggested that up to $30 \%$ of breast cancer survivors develop an anxiety or depressive disorder within a year of diagnosis. On the other hand, some research has demonstrated that women with breast cancer show no difference in psychological distress compared to women with benign breast problems (Montazeri, Harirchi, Vahdami, Khaleghi, Jarvandi, Ebrahimi et al., 2000).

Montazeri et al., (2000) conducted a study with a baseline sample of 168 breast cancer survivors, and a follow-up sample of 151 survivors. The researchers assessed levels of anxiety and depression before diagnosis and three months post-diagnosis using the Hospital Anxiety and Depression Scale (HADS). Results revealed that $48 \%$ had severe symptoms of anxiety at both baseline and follow-up. In contrast to prior research findings, approximately $63 \%$ of survivors had normal scores on the HADS depression subscale both at baseline and follow-up. Both symptoms of anxiety and depression were found to be more prevalent in those who had metastatic disease. At baseline, survivors with breast cancer showed no statistically significant differences in anxiety and depression scores compared to women with benign disease. However, at follow-up, breast cancer survivors had significantly higher mean scores on the HADS for both anxiety $(p<.001)$ and depression $(p<.001)$, compared to those with benign disease. 
Thus, the researchers concluded that, in general, symptoms of anxiety and depression persist three months after disease diagnosis (Montazeri et al., 2000).

Although the above study found that the majority of participants did not experience depressive symptoms, research has suggested that cancer survivors are at increased risk of having co-existent depression. Depression is believed to be particularly common in survivors who experience pain and physical disability. Since pain and physical disability become more common in the terminal phases of cancer, coupled with the threat of impending loss, depression is typical among palliative care patients. In a sample of 63 survivors with advanced cancer, who were currently receiving palliative care services, it was found that $22 \%$ of participants scored above the cutoff for probable depression on the HADS and 25\% scored above the cutoff for probable anxiety. Moreover, anxiety and depression were associated with impairment in cognitive functioning and global health status. This finding continued to be significant after controlling for the effects of pain and illness severity (Smith, Gomm, \& Dickens, 2003). Pain and physical limitations are also more common during treatment compared to posttreatment (although pain can persist as discussed above), with many survivors showing slight and steady improvement in physical and psychological functioning over time (Helgeson, Snyder \& Seltman, 2004), which is why it is important to distinguish between active-treatment and post-treatment survivors.

Variables that are likely related to level of distress in cancer survivors are type of cancer treatment completed and the severity of treatment side effects. Jim, Andrykowski, Munster and Jacobsen (2007) performed a longitudinal, observational study with 151 women who had completed chemotherapy and/or radiotherapy for Stage I or II breast 
cancer. The researchers examined whether the number of physical symptoms/side effects experienced during treatment was a correlate of cancer-related distress and/or general distress, four months after treatment completion, as measured by the Impact of Events Scale and the Mental Health subscale of the Short Form-36. Hierarchical multiple regression analysis (with relevant sociodemographic, clinical, and psychiatric variables entered as controls) revealed that greater physical symptoms/side effects predicted greater total cancer-related distress, intrusive thoughts, and general distress. More specifically, results suggested that physical symptoms/side effects during treatment trigger later cancer-related distress, specifically intrusive thoughts. Intrusive thoughts, in turn, appear to have a negative effect on overall mental health and well-being. The researchers asserted that this finding is important because it suggests that to improve mental health in breast cancer survivors, one must first address intrusive thoughts relating to cancer.

Interestingly, in the above study, physical symptoms/side effects did not significantly predict avoidance. Jim, et al. (2007) made sense of this finding by postulating;

Perhaps patients who are troubled by more physical symptoms/side effects have more intrusive thoughts but also more difficulty avoiding reminders of cancer, whereas patients who have fewer physical symptoms/side effects experience fewer intrusive thoughts and thus do not need to reduce distress by avoiding reminders of cancer. (p. 206)

The researchers suggested that physical symptoms/side effects might lead to increased distress by negatively impacting survivors' self-image and by interfering with important activities, though future research is needed to explore this hypothesis. Overall, it appeared that survivors who experience greater physical symptoms/side effects during 
treatment are at greater risk for later cancer-related distress and, in turn, general distress (Jim, et al., 2007).

\section{Differences Between Cognitions Associated with Anxiety \& Depression}

In 1988, Mitchell and Campbell published a study that sought to examine whether cognitions associated with depression were different than those associated with anxiety. Sixty-four students and administrative staff completed the Beck Depression Inventory, the State-Trait Anxiety Inventory and the Cognitions Questionnaire. While the results of the study are limited due to the fact that the sample was primarily students, it was revealed that individuals with depression scores above the median were significantly more likely to generalize their thoughts from one situation to another and showed greater overall cognitive distortion than individuals with scores below the median. Those who had anxiety scores above the median did not show a greater amount of cognitive distortion, and there was no difference between high and low anxiety scores in terms of generalization from one situation to the next. The researchers concluded that although there are some cognitions that might arise with both anxiety and depression, they represent distinct constructs (Mitchell \& Campbell, 1988).

In general, research suggests that anxiety and depression symptoms are components of a broader construct of negative affectivity, but there are unique components to each type of distress. More specifically, symptoms of anhedonia and low positive affect are relatively unique to depression, and manifestations of somatic tension and arousal are relatively specific to anxiety (Primo, et al., 2000; Watson, Clark, Weber, Assenheimer, Strauss, \& McCormick, 1995). Research has also indicated that those who engage in negative rumination, which is commonly associated with a depressed mood, 
have a more perseverative cognitive style and therefore might not be able to change their cognitive focus despite negative consequences. Worry, consisting of a focus on future potential threat, imagined catastrophes, uncertainties, and risks has also been found to be difficult to dismiss or ignore, and is often perceived as uncontrollable (Segerstrom, Stanton, Alden, \& Shortridge, 2003). When an individual is depressed, for example, his/her thoughts tend to be more negative and self-critical and he/she may experience impaired concentration. When anxious, an individual might attempt to engage in unsuccessful problem solving that is focused on events which have an uncertain outcome (Watkins, 2008). Thus, thoughts can impact the emergence of symptoms of anxiety and depression, and symptoms of anxiety and depression can impact the type of cognitive processing in which an individual engages in. As discussed above, cognitive processing facilitates the emergence of PTG, and therefore it is necessary to assess a survivor's anxiety and depression symptoms as well as cognitive processing when investigating PTG.

\section{The Role of Cognition in Anxiety \& Depression}

Intrusive, unwanted negative thoughts about cancer, accompanied by efforts to avoid these thoughts, are among the more prominent stress-related cognitions experienced by cancer survivors (Primo, Compas, Oppedisano, Howell, Epping-Jordan \& Krag, 2000). These types of cognitions are often associated with symptoms of anxiety and depression. In a study with 85 women with newly diagnosed breast cancer, the majority of whom (61\%) had Stage I cancer, Primo, et al. (2000) found that survivors who reported high levels of both intrusive thoughts and avoidance symptoms (as measured by the Impact of Events Scale), and those high in only intrusive thoughts, had 
the highest levels of anxiety and depression symptoms (as measured by the Symptom Checklist-90-Revised) at the time of diagnosis and at three and six month follow-ups. Moreover, survivors who were high only in avoidance also displayed more anxiety and depression symptoms when compared with survivors who were low in both intrusion and avoidance. When examining demographic variables it was found that younger survivors had higher levels of intrusion, and survivors with fewer years of education had higher levels of avoidance.

The authors speculated that younger survivors might perceive their diagnosis as more threatening as it occurs at a point in their life when a potentially life-threatening illness is more unexpected compared to older survivors. Authors also suggested that survivors with less education may perceive themselves as having fewer resources to cope and thus may rely on avoidance. Interestingly, disease severity did not influence patterns of intrusive thoughts and avoidance; however, it is worth noting that the majority of the sample was comprised of only one disease stage (Primo, et al., 2000). These findings suggest that it is imperative to assess potential anxiety and depression symptoms, as these might be important indicators of current and future distress.

Incorporating the role of meaning in the relationship between cognitions and distress, Vickberg, Bovbjerg, DuHamel, Currie and Redd (2000) conducted a study with breast cancer survivors (two to 15 years past diagnosis), with a variety of disease stages and treatment procedures. The researchers sought to determine if a survivor's sense of global meaning (defined as the belief that one's life has purpose and order, and measured by the Personal Meaning Index of the Life Attitude Profile-Revised) might moderate the relationship between intrusive thoughts and distress. The authors hypothesized, 
More frequent intrusive thoughts would be associated with higher psychological distress among women with lower global meaning. However... among those with higher global meaning, more frequent intrusive thoughts would not be associated with increased distress... we expected that higher global meaning would protect (buffer) those women from the distress such thoughts might otherwise cause. (Vickberg, et al., 2000, p. 153)

Consistent with these hypotheses, hierarchical regression analyses showed a strong association between intrusive thoughts and psychological distress only among those with lower global meaning. For these survivors, more intrusive thoughts were associated with higher psychological distress; yet, this relationship was not found among survivors with higher global meaning. The authors concluded that although global meaning did not prevent intrusive thoughts from occurring, a survivor who feels that life has more purpose and meaning is possibly less distressed by such thoughts. It was also postulated that intrusive thoughts experienced by individuals with high global meaning might be qualitatively different (i.e., less threatening) than those experienced by individuals with low meaning. The authors suggested that future research examine the content of intrusive thoughts to determine whether specific types of thoughts are more distressing than others and whether a sense of meaning influences what types of thoughts a survivor has (Vickberg, et al., 2000).

It is important to note that a sense of meaning and PTG are not the same. Yet, research has suggested that PTG is an outcome of meaning-making (Tedeschi \& Calhoun, 2006). In other words, an individual has to assign some meaning to a traumatic event before they experience growth. This process occurs as an individual is repeatedly exposed to, or reflects on a trauma and begins to have a clearer understanding of the event, typically reappraising the experience by identifying positive aspects of it (Tedeschi \& Calhoun, 2004). Some studies have found that meaning-making processes are related 
to more growth (Bower et al., 1998; Calhoun, Cann, Tedeschi, \& McMillan, 2000; Park \& Ai, 2006).

Putting the key variables of Perceived Threat, Anxiety, Depression, Cognitive Processing and PTG together, the above research demonstrates that at least a moderate level of threat is needed to facilitate growth. If threat is too low or too high, it is probable that growth will not emerge as an individual will likely not have the impetus to engage in the cognitive processing necessary for growth or will be too overwhelmed to engage in deliberate and effortful processing. Given that there is typically some level of on-going distress in those that experience growth, it stands to reason that some survivors will also experience a certain amount of symptoms of anxiety and depression as these often indicate the amount of threat and/or distress an individual feels. Individuals with symptoms of anxiety and depression, would, in turn, likely engage in minimal or negative cognitive processing (defined by the Cognitive Processing of Trauma Scale as Denial and Regret). Subsequently, less growth would likely emerge.

In contrast, if symptoms of anxiety and depression are fairly low, it would then be expected that a survivor would engage in positive cognitive processing (defined by the Cognitive Processing of Trauma Scale as Positive Cognitive Restructuring, Resolution, and Downward Comparison), and, in turn, would experience more PTG. This theorydriven rationale provides the foundation for several hypotheses in this study which will be presented more thoroughly in Chapter Three. 


\section{Types of Cancer}

This study examined PTG in breast, prostate, and colorectal cancer because of their high prevalence rates, occurrence in both females and males, and similar course of treatment.

Breast, prostate, and colorectal cancer represent three of the four (lung cancer is the fourth) leading anatomical sites of both new cancer cases and cancer deaths in Colorado, and the United States as a whole (American Cancer Society, 2008). The American Cancer Society (2008), using a sample of 294,120 men and 271,530 women reported that lung cancer is, by far, the most common fatal cancer in men (31\%), followed by prostate (10\%), and colon \& rectum (8\%). In women, lung (26\%), breast (15\%), and colon \& rectum (9\%) are the leading sites of cancer death. Trends in five-year survival rates based on follow-ups with survivors through 2004, have improved significantly since the 1970 s due, in part, to earlier detection and advances in treatment (American Cancer Society, 2008). Between 1996-2003, prostate cancer had a five-year survival rate of $99 \%$, followed by breast (89\%), rectum (66\%) and colon cancer $(65 \%$; National Cancer Institute, 2007). Also, age of the survivor and stage of the cancer at the time of diagnosis have an impact on survival rates.

From a methodological viewpoint, these cancer types are similar to one another in several ways. First, they all are solid tumor cancers (as opposed to blood cancers) and therefore have similar treatment courses, which typically consist of chemotherapy, radiation, surgery, or a combination, depending upon the specific stage of the cancer (American Cancer Society, 2008). 
Second, according to the National Cancer Institute (2008) breast, prostate, and colorectal cancer all have similar mean ages for initial diagnosis (61, 68, and 71 years old, respectively), although more young people are diagnosed with breast cancer compared to prostate or colorectal cancer. Table 1 presents the percentage of individuals diagnosed with each type of cancer, based on age, using data collected between 2002 and 2006.

Table 1

Percentage of Individuals Diagnosed with Breast, Prostate and Colorectal Cancer Based on Age

\begin{tabular}{|l|l|l|l|}
\hline & Breast & Prostate & Colorectal \\
\hline Under Age 55 & $34.9 \%$ & $9.3 \%$ & $17 \%$ \\
\hline Age 55-84 & $59.5 \%$ & $86 \%$ & $71 \%$ \\
\hline Age 85+ & $5.5 \%$ & $4.7 \%$ & $12.1 \%$ \\
\hline
\end{tabular}

Source: National Cancer Institute, 2008.

Third, a fairly low percentage of individuals diagnosed with these three cancer types will be in Stage IV, although it is anticipated that more colorectal cancer survivors will be compared to breast and prostate. Table 2 presents the percentage of individuals diagnosed with Stage I-IV.

Table 2

Percentage of Individuals Diagnosed with Stage I-IV Cancer

\begin{tabular}{|l|l|l|l|}
\hline & Breast & Prostate & Colorectal \\
\hline Stage I & $60 \%$ & $80 \%$ & $39 \%$ \\
\hline Stage II \& III & $33 \%$ & $12 \%$ & $37 \%$ \\
\hline Stage IV & $5 \%$ & $4 \%$ & $19 \%$ \\
\hline
\end{tabular}

Source: National Cancer Institute, 2008. 
While not many survivors diagnosed with breast, prostate or colorectal cancer will be in an advanced stage of disease, there was potential for a fairly wide range of disease severity to be represented in this study's sample. Therefore, a decision was made to examine the influence of stage of disease on other variables. However, it is important to note that this study examined perceived threat of cancer, and the literature has demonstrated that perceived threat of cancer is associated with PTG beyond objective threat (i.e., stage of disease). Moreover, perceived threat is likely to be related to variables other than disease stage, such as the degree to which beliefs are challenged, etc.

Fourth, both females and males are represented among the diagnoses, which allows for a potentially gender-balanced sample. Although gender differences have not been found in most of the PTG literature to date (Stanton et al., 2006), it is important to consider that the majority of studies are conducted with breast cancer survivors and, therefore, are gender biased.

Finally, given the high prevalence of breast, prostate, and colorectal cancer there was opportunity to recruit a high number of participants, potentially yielding a larger sample size, and subsequently more statistical power. Also, since three types of cancer will be assessed, the results will be more generalizable than if sampling were restricted to a single cancer diagnosis.

Overall, breast, prostate and colorectal cancer have the potential to impact a large number of people, and therefore, it is important that research examine these survivor populations. More specifically, it is imperative that we further our understanding of the mechanisms of PTG among those who experience the most common types of cancer. By doing so, clinical interventions can be created that are not only sensitive to the potential 
for PTG, but which can also help survivors utilize beliefs of growth to promote their adjustment to living with cancer during diagnosis and treatment and beyond.

\section{Summary}

A growing body of literature has examined PTG in the aftermath of trauma. While numerous studies have been conducted investigating PTG among cancer survivors, few have examined the role of cognitive processing in facilitating PTG. The studies that were selected for review provide findings that suggest that cognitive processing, along with perceived threat and an individual's level of anxiety and depression, have the potential to influence the emergence of PTG. However, many of the proposed relationships amongst these variables have been conceptual in nature, and therefore, more research is needed to investigate these issues.

The concept of positive change in the aftermath of trauma has been recognized for centuries, and an emerging body of literature exists on PTG. Yet, there are many unanswered questions regarding the specific mechanisms that facilitate growth. The empirical evidence reported in the literature suggests that not everyone who experiences cancer is going to perceive that they have grown from it, thereby suggesting there are a number of factors that influence PTG. The aim of this study was to develop a regression model to examine factors related to, and predictive of, PTG among cancer survivors. More specifically, Anxiety, Depression, Perceived Threat and Cognitive Processing were explored as predictors of PTG. This regression model was investigated in a group of breast, prostate, and colorectal cancer survivors who had completed primary medical treatment (i.e., chemotherapy, radiation and/or surgery). 


\section{CHAPTER THREE}

\section{METHODS}

\section{Introduction}

The current study examined the role of Anxiety, Depression, Perceived Threat and Cognitive Processing in predicting Posttraumatic Growth (PTG) among breast, prostate, and colorectal cancer survivors. These cancer diagnoses were selected because they represent three of the top four anatomical sites of new cancer cases and cancer deaths in the United States. After reviewing the literature, these particular variables were chosen based upon their common association with cancer and/or their key theoretical function in the facilitation of PTG. Chapter Three will present information regarding participants, power and sample size, measures, procedures, data analysis and the hypotheses of the study.

\section{Participants}

Participants were breast, prostate or colorectal cancer survivors who had completed primary medical treatment (i.e., chemotherapy, radiation, and/or surgery) at least two months prior to the study. This convenience sample was recruited from Rocky 
Mountain Cancer Centers (RMCC) in the Denver metro area. The time period of at least two months post-treatment was chosen because of the assumption that during treatment, survivors are likely overwhelmed with information and the treatment process, including attending various medical appointments and dealing with initial treatment side effects. Therefore, if a survivor is at least two months post-treatment, he/she has presumably started to make the transition from "active patient" to "survivor" and likely has more emotional energy and time to devote to reflecting on his/her cancer experience.

Eligibility criteria for participation in the study were: (1) at least 18 years of age; (2) at least two months post-completion of surgery, chemotherapy, and radiotherapy (can be taking maintenance medication); (3) no history of multiple types of cancer; (4) English speaking. Only individuals who have completed primary treatment, and those with no history of multiple types of cancer, were eligible because people in the midst of cancer treatment, or those with multiple types of cancer, may experience specific psychological symptoms, such as intense feelings of being overwhelmed, that may disrupt cognitive processing and blur the results of the study. Also, those individuals with a history of multiple cancers were excluded in order to create a more homogenous sample. Only data from participants who met eligibility criteria were included in the analyses.

\section{Power and Sample Size}

In order to determine the appropriate sample size needed to achieve statistical power in the current study, a power analysis was conducted using the computer software G*Power 3.1. The analysis was run for the statistical test "Linear Multiple Regression" and was computed with three possible effect sizes $($ small $=0.02$, medium $=0.15$, large $=$ 
0.35, Cohen, 1988) and 11 predictor variables as each regression model included a total of 11 predictors. The power analysis revealed that to achieve statistical power with a small effect size, 1,267 participants would be needed, with a medium effect size, 178 participants would be needed, and with a large effect size, 83 participants would be needed. A medium effect size was targeted for the study as the researcher deemed that a large effect was unlikely and a small effect would be potentially inconsequential in terms of the study's implications. Research has shown that the average response rate for mail surveys is approximately 30\% (Cobanoglu, Warde \& Moreo, 2001); therefore, anticipating an average response rate, and a medium effect size, 600 questionnaires were mailed to potential participants.

\section{Measures}

\section{Demographics}

The survey contained a demographic information section (see Appendix D), which included variables provided by self-report. This information was used for descriptive and analytic purposes and participants were encouraged to complete this section of the survey, which also included seven ethnic categories defined by the federal government as follows: African American, American Indian/Alaskan Native, Asian/Pacific Islander, Caucasian, Hispanic/Latino/a, Multi-racial, and Other.

\section{Perceived Threat}

Perceived Threat was measured with a survey designed for the current study. The measure designed for the study was comprised of items that have been utilized in prior 
research, as well as items created by the researcher (see Appendix E). These items were piloted with five cancer survivors at RMCC before administration to the study participants to ensure the items were clear and understandable and were measuring what they were intended to measure. Two of the Perceived Threat items that have been utilized in previous research (Cordova et al., 2001; Cordova et al., 2007; Salsman et al., 2009) assessed whether cancer constituted a traumatic stressor for the participant. These items were created based upon the Diagnostic and Statistical Manual of Mental Disorders-IVText Revision criteria for PTSD (DSM-IV-TR; American Psychiatric Association, 2000).

Lechner et al. (2003) measured perceived threat in a mixed sample of cancer survivors by asking them to provide a percentage rating of how likely they felt it was that they would die of cancer; a scale ranging from zero to 100 percent was used. The current study utilized three items from the Perceived Threat Questionnaire created by Lechner et al. (2003). While the questionnaire has never been validated for its psychometric properties, the developer of the measure confirmed its use in research (S.C. Lechner, personal communication, April 6, 2009).

Finally, because research has not examined the extent to which being diagnosed with cancer challenges an individual's beliefs about him/herself, others, and the world in general; the researcher created three new items. The items are based on the notion that the more cancer challenges an individual's beliefs, the greater the potential for PTG. Each of the three items asked participants to rate on a 1 ("not at all") to 6 ("extremely") Likerttype scale the extent to which being diagnosed with cancer challenged how the individuals see (1) themselves, (2) others, and (3) the world. For all the items in the 
Perceived Threat questionnaire, higher scores indicate greater threat. The items used in this measure can be found in Appendix E.

\section{Anxiety and Depression}

The Hospital Anxiety and Depression Scale (HADS) was used to measure symptoms of anxiety and depression. The HADS was developed by Zigmond and Snaith in 1983 to identify both possible and probable cases of anxiety disorders and depression among patients in non-psychiatric hospital clinics. The measure is a 14-item questionnaire with two subscales measuring anxiety and depression. Each subscale contains seven items scored on a 4-point Likert-type scale ranging from 0 ("not at all") to 3 ("very much"), indicating degree of psychological distress. The total score for each subscale ranges from zero to 21 and examples of the items include "I feel tense or 'wound up"" and "I still enjoy the things I used to enjoy." In order to prevent "noise" from somatic disorders on the scores, all symptoms of anxiety and depression relating also to physical disorder, such as dizziness, headaches, insomnia, and fatigue were excluded. Symptoms related to serious mental disorders were also excluded since such symptoms were less common in patients attending a non-psychiatric hospital clinic (Bjelland et al., 2002).

Zigmond and Snaith (1983) assessed the internal consistency of the two subscales based on data from 50 medical patients in outpatient clinics, who were dealing with a variety of complaints and illnesses, by calculating correlations between each item and the total score of the remaining items in the subscale. For the anxiety items, the correlations ranged from 0.41 to 0.76 and were all statistically significant $(p<.01)$. The items in the depression scale had correlations ranging from 0.30 to 0.60 and were all statistically 
significant $(p<.02)$. The researchers then conducted analyses to determine what rating for each subscale total score would represent "non-cases," "doubtful" cases and "definite" cases. For both the depression and anxiety scales, it was found that a score of seven or less for "non-cases," scores of eight to 10 for "doubtful" cases, and scores of 11 or more for "definite" cases produced the best results (Zigmond \& Snaith, 1983). These criteria were then applied to another 50 patients and similar results were found. Next, correlations between subscale scores and psychiatric ratings were calculated in order to determine whether scores on the two subscales could also be used as indicators of severity of depression and anxiety, respectively. For both depression and anxiety, statistically significant $(p<.001)$ positive correlations were found $(\mathrm{r}=.70$ and .74 , respectively). The researchers concluded that the subscale scores could justifiably be used as measures of severity of depression and anxiety.

Bjelland et al. (2002) reviewed 747 papers to examine the validity of the HADS. It was found that in most studies an optimal balance between sensitivity and specificity was achieved when "caseness" was defined by a score of eight or above on both subscales. Moreover, correlations between the HADS and other commonly used instruments, such as the Beck Depression Inventory and State Trait Anxiety Inventory, were in the range of .49 to .83 . Overall, the researchers concluded that the HADS performed well in assessing symptom severity and caseness of anxiety disorders and depression in both somatic, psychiatric, and primary care patients and in the general population (Bjelland et al., 2002). The items used in this measure can be found in Appendix F. 
It is worth noting that the HADS has been used with cancer survivors to assess anxiety and depression in various studies (Ho, Chan \& Ho, 2004; Kornblith, Powell, Regan, Bennett, Krasner, Moy, et al., 2007; Lipscomb, et al., 2005; Mager \& Andrykowski, 2002; Thomas, Glynne-Jones, Chait \& Marks, 1997). For instance, a study by Kornblith, et al. (2007) used the HADS as the primary measure of "psychosocial adjustment" in a group of breast and endometrial cancer survivors who were on average 3.7 years post-treatment. A study with a Chinese sample of 32 male and 156 female cancer survivors, who were at least five years "disease free," used the Chinese version of the HADS to assess symptoms of anxiety and depression and found adequate internal reliability values for the anxiety and depression subscales ( 0.88 and 0.78 , respectively; Ho, et al., 2004). Finally, Thomas, et al. (1997) utilized the HADS to assess anxiety in a group of 65 cancer survivors, two-thirds of whom had completed treatment approximately ten years prior. Thus, the HADS has been shown to be an appropriate anxiety and depression measure for use with cancer survivors who are actively in treatment as well as post-treatment survivors.

\section{Cognitive Processing}

The Cognitive Processing of Trauma Scale (CPOTS; Williams et al., 2002) is a 17-item scale measuring cognitive processing of traumatic experiences. Each item was scored on a Likert-type response scale ranging from -3 ("strongly disagree") to +3 ("strongly agree"). The CPOTS measures five aspects of cognitive processing: (a) Positive Cognitive Restructuring, (b) Downward Comparison, (c) Resolution, (d) Denial, and (e) Regret. Examples of items are: "There is ultimately more good than bad in this 
experience" and "I have figured out how to cope." The instrument is scored by adding +3 to each item score, and then computing a mean score for each of the five subscales.

The CPOTS was developed using two different college student samples. Based on initial testing with 35 participants, the researchers conducted a second study with 229 undergraduate students in order to refine the measure. The researchers investigated the factor structure of the scale utilizing multiple confirmatory factor analysis, and the results indicated that a five factor model was the best fit. The five factors in the final model were: (a) Positive Cognitive Restructuring (three items, $\alpha=$ .83), (b) Resolution/Acceptance (four items, $\alpha=.81$ ), (c) Downward Comparison (three items, $\alpha=.72$ ), (d) Denial (four items, $\alpha=.85$ ), and (e) Regret (three items, $\alpha=.74$; Williams et al., 2002). A sub-sample of 67 participants completed the measure four weeks after the first administration to assess test-retest reliability. Correlations between each of the subscales administered at baseline and four weeks later ranged from $r=.70$ to .85 ; all were significant at $p<.001$. These results suggest that the subscales of the CPOTS have adequate test-retest reliability (Williams et al., 2002). Correlations among each of the five subscales revealed that the subscales indicative of positive cognitive processing (i.e., Positive Cognitive Restructuring, Resolution, and Downward Comparison) are negatively associated with those subscales that indicate minimal/negative cognitive processing (i.e., Denial, Regret). Further, the subscales indicating positive cognitive processing are positively associated with each other, as are the two subscales indicating minimal cognitive processing (Williams et al., 2002). These findings suggest that it may be appropriate to create two composite variables from the five subscales, Positive and Negative Cognitive Processing, when analyzing data. 
The CPOTS has also been correlated with the Impact of Event Scale (IES) and the Stress-Related Growth Scale (SRGS) to establish construct validity. It was found that the IES items that are indicative of relatively little cognitive processing were positively associated with Denial and Regret ( $r$ values ranged from .24 to .51 , all $p$ values $<.001$ ) and negatively associated with Resolution, Positive Cognitive Restructuring and Downward Comparison ( $r$ values ranged from -.16 to -.54 , all $p$ values $<.01$ ). Further, Positive Cognitive Restructuring was associated with the SGRS $(r=.31, p<.001)$, while Denial and Regret were minimally associated with SRGS. Thus, when compared to other validated measures, the subscales of the CPOTS appear valid. Overall, the researchers reported that the CPOTS is a "17-item psychometrically solid measure" (Williams et al., 2002, p. 356). The items used in this measure can be found in Appendix G.

\section{Posttraumatic Growth}

The Posttraumatic Growth Inventory (PTGI; Tedeschi \& Calhoun, 1996) was used to measure the extent to which cancer survivors perceived personal benefits, including changes in perceptions of self, relationships with others, and philosophy of life, accumulated from their attempts to cope with cancer and its aftermath. The PTGI is a 21item self-report measure scored on a 6-point Likert-type scale ranging from 0 ("I did not experience this change as a result of my crisis") to 5 ("I experienced this change to a very great degree as a result of my crisis"). Scores can range from zero to 105, with high scores indicating positive growth. Examples of items are: "I changed my priorities about what is important in life" and "I have a greater appreciation for the value of my own life." The scale can be used with various types of trauma as it was developed to measure 
positive outcomes after traumatic experiences. In the current study, "my crisis" was changed to, "my cancer."

The PTGI has five factors: Relating to Others, New Possibilities, Personal Strength, Spiritual Change, and Appreciation of Life, which are based on principle component analyses performed with data from 604 undergraduate students. Relating to Others includes positive changes such as developing stronger bonds with loved ones, reestablishing relationships with family members/friends, or gaining more compassion for others. New Possibilities refers to changes such as making choices in a more conscious manner according to a plan, or changing factors in one's life that one believes need changing. Personal Strength may be expressing greater self-reliance and feeling more able to accept circumstances and developing personal strength that may help one through future challenges. Spiritual Change includes re-evaluating spiritual beliefs, associating with a community of similar believers, or connecting with spiritual roots. Lastly, Appreciation of Life refers to changes such as trying to live each day more fully, rethinking one's values and priorities about what is most important in life, and acting differently regarding changed priorities (Tedeschi \& Calhoun, 1996).

Tedeschi and Calhoun (1996) reported that the internal consistency of the PTGI is $\alpha=.90$. Research has demonstrated that all items contribute relatively equally to the consistency of the scale. The internal consistency of each factor is as follows: New Possibilities $(\alpha=.84)$; Relating to Others $(\alpha=.85)$; Personal Strength $(\alpha=.72)$; Spiritual Change $(\alpha=.85)$; and Appreciation of Life $(\alpha=.67)$. The correlations among the factors ranged from .27 to .52 , and the correlations of the factors with the PTGI total score ranged from .62 to .83 , which indicates some separate contributions by these factors 
(Tedeschi \& Calhoun, 1996). Overall, test-retest reliability over the period of two months, with 28 participants, was acceptable at $r=.71$ (Tedeschi \& Calhoun, 1996).

It is interesting to note that Tedeschi and Calhoun (1996) found that in a subsample of 117 undergraduate participants, women reported more benefits than men (F $(1,113)=10.69, p<.001)$ and people experiencing severe trauma reported more benefits than those who did not $(\mathrm{F}(1,113)=12.33, p<.001)$. In general, the researchers concluded that the PTGI has sound internal consistency, acceptable test-retest reliability, and that among people reporting a variety of life difficulties, scores on the scale are approximately normally distributed (Tedeschi \& Calhoun, 1996). The items used in this measure can be found in Appendix H.

\section{Procedure}

Permission to conduct this study was granted by the Institutional Review Board for the use of Human Subjects at the University of Denver. Additionally, permission was obtained from the Institutional Review Board utilized by the Rocky Mountain Cancer Centers (RMCC) of the Denver metro area (HealthONE). Two RMCC employees, Teri Simoneau, PhD and Susan Ash-Lee, MSW, LCSW, were identified on the IRB protocol as "co-investigators" of the study. This was done because these professionals meet the Health Insurance Portability and Accountability Act (HIPAA) requirements that allow them access to survivor identifying information through the RMCC database. Therefore, Dr. Simoneau was the primary professional, with the help of Susan Ash-Lee, who identified potential participants in the database and mailed them a questionnaire packet. The researcher did not have access to or knowledge of, a participant's identifying 
information until he/she consented to participating in the study and mailed the researcher a signed informed consent form and HIPAA form along with a completed questionnaire.

The RMCC database holds the names of thousands of cancer survivors. In order to identify potential participants, Dr. Simoneau and Susan Ash-Lee requested the names and addresses of all survivors with a diagnosis of breast, prostate or colorectal cancer who were seen in the Denver Division clinics, and whose first appointment at RMCC was between the dates of 1/1/06 and 9/1/09 from the database coordinator. The specific time frame of between 1/1/06 to 9/1/09 was chosen in order to limit the potential list of participants and to identify survivors who had completed primary treatment within the last few years. A list of 4,474 survivors was yielded, which was broken down as follows: Breast $=3,956$, Prostate $=147$, Colorectal $=367$. All prostate survivors were sent a questionnaire packet as the number was so low, and the remaining packets (433) were split generally evenly between breast (221) and colorectal (232) cancer survivors.

To narrow down the 3,956 identified breast cancer survivors, all survivors who had Carcinoma in Situ were removed (a total of 461 survivors), because that is a low risk breast cancer, and then the first 221 were chosen alphabetically. Similarly, with colorectal cancer survivors the first 232 were chosen alphabetically. Dr. Simoneau postulated that the reason there were so few prostate cancer survivors on the database list is because they are often treated by urologists, not oncologists, and typically only come to the attention of oncologists when the disease is metastatic (T. Simoneau, personal communication, April 19, 2010).

The questionnaire packets contained two copies of an informed consent form, two copies of a HIPAA form (participants were advised to retain one copy of each form for 
their reference), a refusal postcard, and the self-report measures mentioned previously. The informed consent form provided potential participants with information regarding the purpose of the study, including the risks and benefits, and highlighted that participation was voluntary. The confidentiality of participant responses was emphasized and assured on the form and all participants were discouraged from providing any information on the questionnaires that might have led to potential identification. Consent to participate in the study was provided when participants returned a signed copy of the informed consent and HIPAA form, along with the completed questionnaire, to the researcher. If a participant decided not to participate, he/she was asked to return the postage-paid pre-addressed refusal postcard to the researcher. The completion time of the questionnaire was estimated at 20-30 minutes based upon piloting the entire questionnaire with five cancer survivors at RMCC.

The researcher compiled and placed postage on each of the 600 questionnaire packets and dropped off the packets and follow-up mailings (see below) to Dr. Simoneau. Each questionnaire was numbered in the upper right hand corner of the cover page and on the corner of each outside envelope. These numbers corresponded to a number next to the participant's name on a mailing list in order to track receipt of completed questionnaires. The mailing list was kept and tracked by Dr. Simoneau so that the researcher did not have access to participants' identifying information. Dr. Simoneau assigned each potential participant a number, addressed the packets and mailed them to the participants. As the researcher received refusal postcards and/or returned questionnaire packets, she emailed Dr. Simoneau with the identification numbers so that the individuals could be marked off the mailing list and would not receive follow-up mailings. 
As reported previously, mail surveys have an expected response rate of approximately $30 \%$. This study used the follow-up mailing procedure outlined by Dillman (2000) in order to increase the response rate. There were three follow-up mailings, which were all mailed by Dr. Simoneau. The first letter was mailed one week after the original mailing and was sent to all potential participants. It served as both a thank you for those who had responded and as a courteous reminder for those who had not. Three weeks after the original mailing, a second letter was sent only to nonrespondents (those who had not returned the refusal postcard or a completed questionnaire). This letter informed non-respondents that their questionnaire, or refusal postcard, had not been received, and appealed for a return. If participants were in need of another questionnaire, they were instructed to contact Dr. Simoneau or Susan Ash-Lee at RMCC. Five weeks after the original mailing a final reminder letter was sent to all nonrespondents that contained the same information as the second letter (Dillman, 2000).

\section{Data Analyses}

The alpha level was set at $p<.05$ for all statistical analyses. A cross-sectional design was used. Four hierarchical regression analyses were conducted to explore the potential effect of both Positive and Negative Cognitive Processing on the relationship between Anxiety, Depression, Perceived Threat and PTG. The regression assumptions of normality, linearity, independence, multicollinearity, and homoscedasticity were determined.

\section{Preliminary Analyses}

Preliminary analyses were conducted for several purposes. First, data were collected from several RMCC sites throughout the Denver metro area and it was important to 
ensure, using descriptive statistics and Analysis of Variance (ANOVA), that the samples were similar enough to justify combining them for future analyses. Second, data were collected from persons with cancer of one of three specific anatomical sites and as in the example above, it was important to determine whether or not the samples could be combined. Third, a small portion of the data set (13\%) had experienced a recurrence of cancer and it was necessary to determine whether data from those with and without a cancer recurrence could be combined. Fourth, because several items were created and added to the Perceived Threat questionnaire, analyses were performed to refine and yield a reliable instrument. Finally, analyses were conducted on the five subscales of the Cognitive Processing of Trauma Scale in order to determine if the subscales could be grouped together to form the two composite variables of Positive (i.e., Positive Cognitive Restructuring, Downward Comparison, Resolution) and Negative (i.e., Denial, Regret) Cognitive Processing.

\section{Primary Analyses}

Several demographic variables were used as control variables in each regression equation. Research has shown that gender differences may exist in the level of PTG reported, with women being more likely to report PTG than men (Tedeschi \& Calhoun, 1996). Therefore, Gender was controlled for in the regression models. Stage of disease may also influence the amount of PTG reported, with more severe stages, and the accompanying greater threat to mortality, facilitating more growth (Tomich \& Helgeson, 2004). Thus, Stage of Disease was included as a demographic variable. Time since treatment completion has been found to be related to PTG, such that greater PTG emerges over time (Helgeson, Reynolds, \& Tomich, 2006). Therefore, Time Since 
Treatment Completion was included. Finally, younger age has sometimes been associated with greater reports of PTG (Stanton et al., 2006). Therefore, Age was included in the regression model. It was also suggested (C. Parry, personal communication, June 15, 2009) that the specific cancer treatment a survivor received, possible permanent aftereffects of treatment, and the amount that after-effects interfere with a survivor's daily life may influence the amount of PTG that emerges and, therefore, need to be included in the regression model. Thus, analyses were performed to examine if these specific variables had statistically significant relationships with the main outcome variables (see Chapter 4 for more information).

The study hypotheses were as follows:

1. Anxiety, Depression, and Perceived Threat will predict Positive Cognitive Processing over and beyond the contribution of the demographic control variables. It is hypothesized that Anxiety and Depression will negatively predict Positive Cognitive Processing, while Perceived Threat will positively predict Positive Cognitive Processing.

The research reviewed in Chapter Two demonstrated that a sufficient level of threat is needed to facilitate any type of cognitive processing. Thus, in general, it is predicted that Perceived Threat would be positively associated with Positive Cognitive Processing. In contrast, an individual experiencing symptoms of anxiety and depression would likely engage in minimal, or no positive cognitive processing due to potentially experiencing a high degree of worry and non-deliberate ruminative, rather than reflective thinking. Therefore, negative relationships between Anxiety and Depression and Positive Cognitive Processing are predicted. 
Hierarchical regression analyses were utilized to investigate the relative contributions of Anxiety, Depression, and Perceived Threat in the prediction of Positive Cognitive Processing. The following participant characteristics were included in the first block of variables: Age, Gender, Stage of Diagnosis, and Time Since Treatment Completion. Anxiety and Depression were entered in the second block, while Perceived Threat was entered in the third block. Positive Cognitive Processing was entered as the dependent variable.

2. Positive Cognitive Processing will significantly predict PTG over and beyond the demographic control variables, Anxiety, Depression, and Perceived Threat. In other words, Positive Cognitive Processing will account for a significant amount of the variance of PTG beyond the demographic control variables (i.e., Age, Gender, Stage of Diagnosis, Time Since Treatment Completion), Anxiety, Depression, and Perceived Threat. It is hypothesized that Positive Cognitive Processing and Perceived Threat will positively predict PTG, while Anxiety and Depression will negatively predict PTG.

It has been argued that threat, along with deliberate and effortful cognitive processing (what the CPOTS defines as Positive Cognitive Processing), facilitates the emergence of PTG. Therefore, positive relationships between these variables and growth are anticipated. On the other hand, it is expected that Anxiety and Depression will be negatively associated with growth due to the assumption that individuals experiencing these symptoms would likely not engage in deliberate and effortful thinking.

A hierarchical multiple regression analysis was utilized to investigate the relative contributions of Anxiety, Depression, Perceived Threat, and Positive Cognitive Processing in the prediction of PTG. The following participant characteristics were 
included in the first block of variables: Age, Gender, Stage of Diagnosis, and Time Since Treatment Completion. Anxiety and Depression were entered in the second block. Perceived Threat was entered in the third block, and then Positive Cognitive Processing was entered in the fourth block. PTG scores were entered as the dependent variable.

3. Anxiety, Depression, and Perceived Threat will predict Negative Cognitive Processing over and beyond the contribution of the demographic control variables. It is hypothesized that Anxiety, Depression, and Perceived Threat will all positively predict Negative Cognitive Processing.

It is anticipated that Perceived Threat will positively predict Negative Cognitive Processing based on the theoretical assumption stated above that a certain level of threat and/or subjective impact is needed to prompt cognitive processing of any type. Stated another way, an individual who engages in negative cognitive processing (what the CPOTS defines as Denial and/or Regret) would have likely experienced his or her cancer as threatening. Furthermore, an individual who is experiencing symptoms of anxiety and depression is more likely to engage in negative types of thinking compared to positive types. Thus, positive relationships are also anticipated between Anxiety and Depression and Negative Cognitive Processing.

Hierarchical regression was utilized to investigate the relative contributions of Anxiety, Depression, and Perceived Threat in the prediction of Negative Cognitive Processing. The following participant characteristics were included in the first block of variables: Age, Gender, Stage of Diagnosis, and Time Since Treatment Completion. Anxiety and Depression were entered in the second block, and then Perceived Threat was 
entered in the third block. Negative Cognitive Processing was entered as the dependent variable.

4. Negative Cognitive Processing will significantly predict PTG over and beyond the demographic control variables, Anxiety, Depression, and Perceived Threat. In other words, Negative Cognitive Processing will account for a significant amount of the variance of PTG beyond the demographic control variables (i.e., Age, Gender, Stage of Diagnosis, Time Since Treatment Completion), Anxiety, Depression, and Perceived Threat. It is hypothesized that Negative Cognitive Processing, Anxiety, and Depression will negatively predict PTG, while Perceived Threat will positively predict PTG.

This hypothesis is based on the same theoretical basis as above. Anxiety and Depression are anticipated to have a negative association with PTG, because an individual with these symptoms expectedly engages in negative cognitive processing. Negative Cognitive Processing, in turn, is expected to have a negative relationship with PTG because this type of processing is likely intrusive, non-deliberate, non-effortful and non-reflective in nature, and thus, would not facilitate growth. Finally, Perceived Threat is, again, expected to be positively related to PTG.

A hierarchical multiple regression analysis was utilized to investigate the relative contributions of Anxiety, Depression, Perceived Threat, and Negative Cognitive Processing on the prediction of PTG. The following participant characteristics were included in the first block of variables: Age, Gender, Stage of Diagnosis, and Time Since Treatment Completion. Anxiety and Depression were entered in the second block. Perceived Threat was entered in the third block, and then Negative Cognitive Processing was entered in the fourth block. PTG scores were entered as the dependent variable. 


\section{Summary}

The role of Anxiety, Depression, Perceived Threat, and Cognitive Processing in facilitating PTG was examined amongst breast, prostate and colorectal cancer survivors who had completed primary medical treatment. All data were collected through selfreport measures (i.e., demographic questionnaire, Perceived Threat questionnaire, Hospital Anxiety and Depression Scale, Cognitive Processing of Trauma Scale, and Posttraumatic Growth Inventory) which were mailed to the homes of potential participants. In order to better understand the potential mechanisms of PTG, four primary hierarchical regression analyses were conducted. Two of the analyses explored the predictive role of Anxiety, Depression, and Perceived Threat related to either Positive or Negative Cognitive Processing, while the other two analyses explored the association of these variables, including Cognitive Processing, to PTG. 


\section{CHAPTER FOUR}

\section{RESULTS}

\section{Overview}

In this chapter, the findings of the statistical analyses associated with the study are presented. Offered are the results of the preliminary analyses followed by the results of the primary analyses related to the four main hypotheses. All preliminary, primary and follow-up statistical analyses were performed using the Statistical Package for the Social Sciences version 18.0 (SPSS 18.0). All statistical procedures used two-tailed tests of significance with the alpha level set at $p<.05$.

\section{Preliminary Analyses}

This portion of the chapter includes: 1) details of the survey response rate, 2) an analysis of missing data and how it was treated in analyzing the research hypotheses, 3) the participants' demographic and cancer information, 4) results of reliability analyses for the main study variables: Anxiety, Depression, Life Outlook Threat, Physical Threat, Positive Cognitive Processing, Negative Cognitive Processing, and PTG 5) descriptive statistics and correlations related to the main variables analyzed in the research hypotheses, 6) results of analyses (ANOVAs) to examine possible differences between 
three sets of groups (no recurrence of cancer/recurrence of cancer, single cancer treatment received/multiple cancer treatments received, and no permanent after-effects of treatment/permanent after-effects of treatment), an ANOVA to examine differences between three groups (breast, prostate and colorectal cancer survivors), as well as an ANOVA to examine differences between groups based upon location of treatment, and 7) results of one additional ANOVA to examine differences between four groups based upon the degree after-effects interfere with a survivor's daily life (none, some, a little, a lot).

\section{Survey Details and Response Rate}

This study used a confidential mail survey method. Breast, prostate and colorectal cancer survivors treated at one of several Rocky Mountain Cancer Centers (RMCC) Denver metro area locations were invited to participate in the survey $(n=600)$. Out of the 600 potential participants 211 returned questionnaires, a response rate of $35 \%$. One hundred sixty nine $(n=169)$ of the returned questionnaires included signed consent and HIPAA forms and met eligibility criteria. It is important to note that 22 of the 169 participants had experienced a recurrence of cancer. In order to determine if survivors who had a recurrence of cancer were statistically similar to those who had not, a preliminary analysis was conducted (please see below for more information). There were 42 returned questionnaires that were not eligible for use in data analysis. Of the 42 , nine survivors did not return a signed consent and/or HIPAA form, 11 had a history of multiple types of cancer, 10 survivors were still in primary cancer treatment, and two provided unclear answers, which raised the possibility that their responses were not valid. 
Furthermore, 10 individuals were survivors of cancers other than breast, prostate or colorectal. Therefore, the total sample size used in data analyses was 169 .

One hundred thirty-three potential participants declined to participate in the study. One hundred sixteen of these individuals returned the refusal postcard, 10 returned the entire blank questionnaire packet, and seven survivors left phone messages for the researcher. Finally, 15 individuals contacted the researcher via note on the refusal postcard, hand-written note, or phone message explaining that the potential participant had passed away.

Analysis of Missing Data

There were 169 completed surveys in the final data set. As part of the preliminary analyses, the data set was examined using Frequencies analyses to assess the missing data in order to understand possible patterns or reasons that might help to explain why data were missing. Forty-eight completed surveys were missing some type of data. Out of these 48, 18 questionnaires were missing demographic information only, three participants appeared to have accidentally skipped an entire page of the questionnaire packet, and the remainder $(n=27)$ missed certain items on one of several measures within the questionnaire packet. An inspection of the missing data on these cases was completed by visually exploring the database and making note of the demographic and cancer history characteristics of each participant. No discernable pattern emerged. Missing values did not appear to be related to age, gender, race/ethnicity, marital status, living arrangement, education level, income, type of cancer, stage of cancer, type of treatment received, time since completed treatment, or recurrence of cancer. Thus, data were considered to be missing at random (Tabachnick \& Fidell, 2007). It is also 
important to note, that although 48 questionnaires were missing some type of data, for the variables utilized in primary analyses, the maximum number of cases missing data was nine. This was the case for the variables of Stage of Diagnosis and Location of Treatment. All other variables used in analyses had four or fewer cases of missing data, with five variables missing no data. Given the sample size, and due to the random nature of the missing values, it was determined that it was acceptable to not control for or correct missing data in the statistical analyses.

\section{Demographic Information}

A demographic questionnaire (Appendix C) designed for this study was used to collect information on the participants' demographic characteristics as well as cancer diagnosis and treatment. Results are presented in Table 3 and Table 4 . The demographic variables initially used in the analyses were Age, Gender, Stage of Diagnosis, and Time Since Treatment Completion. The results indicated that the sample was relatively homogeneous related to these variables.

Table 3

Overview of Demographic Characteristics

\begin{tabular}{|c|c|c|c|}
\hline \multicolumn{2}{|c|}{ Variable } & Frequency & Percentage \\
\hline \multicolumn{2}{|c|}{ Total Participants } & 169 & 100 \\
\hline Age Range & $\mathrm{M}(\mathrm{SD})$ & & \\
\hline 18 to 29 & $61.24(11.38)$ & 0 & 0 \\
\hline 30 to 39 & & 2 & 1.2 \\
\hline 40 to 49 & & 25 & 14.9 \\
\hline 50 to 59 & & 47 & 27.9 \\
\hline 60 to 69 & & 56 & 33.4 \\
\hline 70 to 79 & & 27 & 16.1 \\
\hline 80 to 89 & & 10 & 5.3 \\
\hline $90+$ & & 1 & 0.6 \\
\hline
\end{tabular}




\begin{tabular}{|c|c|c|}
\hline Variable & Frequency & Percentage \\
\hline \multicolumn{3}{|l|}{ Gender } \\
\hline Female & 117 & 69.2 \\
\hline Male & 52 & 30.8 \\
\hline \multicolumn{3}{|l|}{ Race/Ethnicity } \\
\hline Caucasian & 159 & 94.1 \\
\hline Hispanic, Latino/a & 4 & 2.4 \\
\hline African-American & 3 & 1.8 \\
\hline Asian or Pacific Islander & 2 & 1.2 \\
\hline American Indian or Alaskan Native & 0 & 0 \\
\hline Other & 1 & 0.6 \\
\hline \multicolumn{3}{|l|}{ Marital Status } \\
\hline Single (never married) & 8 & 4.7 \\
\hline Committed relationship & 5 & 3.0 \\
\hline Married/Remarried & 118 & 69.8 \\
\hline Divorced/Separated/Widowed & 37 & 21.9 \\
\hline Other & 1 & 0.6 \\
\hline \multicolumn{3}{|l|}{ Living Arrangement } \\
\hline Live alone & 29 & 17.2 \\
\hline Live with spouse/partner, w/children & 40 & 23.7 \\
\hline Live with spouse/partner, w/out children & 81 & 47.9 \\
\hline Live with children & 11 & 6.5 \\
\hline Live with someone else & 4 & 2.4 \\
\hline Other & 3 & 1.8 \\
\hline \multicolumn{3}{|l|}{ Education (\# of years) $\mathrm{M}$ (SD) } \\
\hline $16.08(2.28)$ & 1 & 0.6 \\
\hline 9 & 0 & 0 \\
\hline 10 & 1 & 0.6 \\
\hline 11 & 1 & 0.6 \\
\hline 12 & 16 & 9.5 \\
\hline 13 & 8 & 4.7 \\
\hline 14 & 11 & 6.5 \\
\hline 15 & 9 & 5.3 \\
\hline 16 & 47 & 27.8 \\
\hline 17 & 24 & 14.2 \\
\hline 18 & 23 & 13.6 \\
\hline $19+$ & 27 & 16.0 \\
\hline
\end{tabular}




\begin{tabular}{lcc}
\hline \multicolumn{1}{c}{ Variable } & Frequency & Percentage \\
\hline Household Income & & \\
Under $\$ 25,000$ & 10 & 5.9 \\
$\$ 25,001-\$ 50,000$ & 26 & 15.4 \\
$\$ 50,001-\$ 75,000$ & 30 & 17.8 \\
$\$ 75,001-\$ 100,000$ & 36 & 21.3 \\
$\$ 100,000+$ & 60 & 35.5 \\
\hline
\end{tabular}

Table 4

Overview of Cancer Diagnosis and Treatment Information

\begin{tabular}{|c|c|c|}
\hline Variable & Frequency & Percentage \\
\hline Total Participants & 169 & 100 \\
\hline \multicolumn{3}{|l|}{ Type of Cancer } \\
\hline Breast & 84 & 49.7 \\
\hline Prostate & 24 & 14.2 \\
\hline Colorectal & 61 & 36.1 \\
\hline Female & 33 & 54.1 \\
\hline Male & 28 & 45.9 \\
\hline \multicolumn{3}{|l|}{ Stage of Cancer (at Initial Diagnosis) } \\
\hline Pre-Cancer/Stage 0 & 3 & 1.8 \\
\hline Stage I & 49 & 29.0 \\
\hline Stage II & 57 & 33.7 \\
\hline Stage III & 30 & 17.8 \\
\hline Stage IV & 14 & 8.3 \\
\hline Stage V & 2 & 1.2 \\
\hline Other & 5 & 3.0 \\
\hline \multicolumn{3}{|l|}{ Location of Treatment ( $>5 \%$ of sample) } \\
\hline RMCC Midtown & 25 & 14.8 \\
\hline RMCC Rose & 35 & 20.7 \\
\hline RMCC Skyridge & 15 & 8.9 \\
\hline All other locations & 85 & 50.3 \\
\hline \multicolumn{3}{|l|}{ Treatment Received } \\
\hline Single Cancer Treatment Received & 49 & 29.0 \\
\hline Multiple Cancer Treatments Received & 120 & 71.0 \\
\hline
\end{tabular}




\begin{tabular}{|c|c|c|}
\hline Variable & Frequency & Percentage \\
\hline \multicolumn{3}{|c|}{ Time Since Completed Treatment } \\
\hline $2-4$ months & 8 & 4.7 \\
\hline $5-6$ months & 10 & 5.9 \\
\hline $7-12$ months & 17 & 10.1 \\
\hline $13-36$ months & 55 & 32.5 \\
\hline $37-60$ months & 26 & 15.4 \\
\hline $61-84$ months & 18 & 10.7 \\
\hline $85-108$ months & 10 & 5.9 \\
\hline $109-132$ months & 8 & 4.7 \\
\hline $133-156$ months & 6 & 3.6 \\
\hline $157-180$ months & 5 & 3.0 \\
\hline $180+$ months & 5 & 3.0 \\
\hline Unknown & 1 & 0.6 \\
\hline \multicolumn{3}{|l|}{ Recurrence of Cancer } \\
\hline Yes & 22 & 13.0 \\
\hline No & 147 & 87.0 \\
\hline \multicolumn{3}{|c|}{ Number of Recurrences } \\
\hline $1-2$ & 17 & 10.1 \\
\hline More than 2 & 3 & 1.8 \\
\hline Other & 1 & 0.6 \\
\hline \multicolumn{3}{|c|}{ Permanent After-Effects from Treatment } \\
\hline Yes & 90 & 53.3 \\
\hline No & 76 & 45.0 \\
\hline \multicolumn{3}{|c|}{ Degree After-Effects Interfere w/Daily Life } \\
\hline None & 20 & 11.8 \\
\hline A little & 28 & 16.6 \\
\hline Some & 30 & 17.8 \\
\hline A lot & 9 & 5.3 \\
\hline
\end{tabular}




\section{Reliability Analyses Related to Main Variables}

The Perceived Threat questionnaire used in this study contained three items created by the researcher that have never been used in prior research. The three items assessed various aspects of how being diagnosed with cancer challenged a survivor's life outlook and are found under the heading of Life Outlook in Appendix E. The remaining five items in the scale have been used in prior research (Lechner, 2003a, 2009b; Salsman, 2009), and measured physical threat related to cancer diagnosis. These items can be found under the two headings of Physical Threat and Reaction to Cancer. It is worth noting that although there are two separate headings all five items are related to physical threat. Based on theory (Janoff-Bulman, 1992; Tedeschi \& Calhoun, 2004) the researcher believed that the Life Outlook items were theoretically different than the Physical Threat items as the former were developed to measure cognitive threat rather than physical threat. Therefore, the researcher thought it appropriate to create two separate Perceived Threat variables: Life Outlook Threat and Physical Threat. In order to determine if the scale was reliable, reliability analyses using SPSS 18.0 were performed. More specifically, the internal consistency of the scale was assessed using Cronbach's coefficient alpha, which provides an indication of the average correlation among the items of the scale. Values range from zero to one, with higher values indicating greater reliability (Pallant, 2007). Nunnally (1978) recommended that a minimum level of .7 is needed to establish adequate reliability. Results of the reliability analysis indicated that the overall scale, consisting of eight items, had an estimated reliability of .87 . The three items comprising Life Outlook Threat had a Cronbach's alpha of .90 , which indicated that all of the items are essentially measuring the same 
construct. Finally, the five items that comprise Physical Threat had a Cronbach's alpha of .83 , again indicating that all of the items are essentially measuring the same construct. Based on these results, the researcher determined it was appropriate to run subsequent analyses with two Perceived Threat variables: Life Outlook Threat and Physical Threat.

Next, reliability analyses were conducted for the Anxiety and Depression subscales of the Hospital Anxiety and Depression Scale (HADS). Results indicated that the seven items comprising the Anxiety subscale of the HADS had an estimated reliability of .85 , while the seven items comprising the Depression subscale had an estimated reliability of .82 . Therefore, based on these results, the measure was reliable.

Reliability analyses were also performed to determine if it was appropriate to examine two subscales of the Cognitive Processing of Trauma Scale (CPOTS): Positive Cognitive Processing and Negative Cognitive Processing rather than examining each of the five subscales separately. The researcher speculated that positive and negative cognitive processing would differentially influence the amount of PTG that is reported by survivors, and therefore having two subscales assessing opposite ends of the spectrum was key to the study hypotheses. Ten items comprise the subscales that are theorized to represent Positive Cognitive Processing (i.e., Positive Cognitive Restructuring, Resolution, and Downward Comparison). Results revealed that these 10 items have a Cronbach's alpha of .83. One item, "Other people have had worse experiences than mine," which is part of the Downward Comparison subscale, had a low corrected item-total correlation of .236 (values below .3 are questionable). This result suggested that this item might be measuring something different from the other items as a whole. However, given the high overall alpha level, the item was retained. Seven items 
comprise the subscales that form Negative Cognitive Processing (i.e., Denial and Regret). The items had an alpha of .70, with all items having corrected item-total correlations of .33 or greater; thus, all items were retained. Overall, the results suggested that it was appropriate to divide the CPOTS into two subscales assessing Positive and Negative Cognitive Processing. Finally, a reliability analysis was performed on the Posttraumatic Growth Inventory (PTGI). The 21 items had an alpha of .96, indicating that all items are essentially measuring the same construct.

\section{Descriptive Statistics for Independent and Dependent Variables}

Descriptive analyses of the independent and dependent variables included in the study were conducted to determine if the responses were normally distributed and if the data showed adequate variability within this sample of breast, prostate and colorectal cancer survivors (see Table 5). An examination of the data indicated that the responses were normally distributed and that there was adequate variability within the sample for the variables of Life Outlook Threat, Physical Threat, and Posttraumatic Growth. However, the distributions of Anxiety, Depression, and Negative Cognitive Processing were positively skewed with the majority of scores clustering around lower values. In contrast, the distribution of Positive Cognitive Processing was negatively skewed with the majority of scores clustering around higher values. Thus, there was a lack of variability within the sample for these variables. 
Table 5

Descriptive Statistics for Independent and Dependent Variables

\begin{tabular}{|c|c|c|c|c|c|c|}
\hline Variable & $\mathrm{N}$ & Mean & SD & Min & Max 1 & $\begin{array}{l}\text { Possible } \\
\text { Range }\end{array}$ \\
\hline \multicolumn{7}{|l|}{ Independent Variables } \\
\hline Anxiety (HADS) & 167 & 4.72 & 3.83 & 0 & 18 & $0-21$ \\
\hline Depression (HADS) & 167 & 2.88 & 3.18 & 0 & 16 & $0-21$ \\
\hline Life Outlook Threat & 167 & 10.23 & 4.68 & 3 & 18 & $3-18$ \\
\hline Physical Threat & 166 & 16.62 & 6.26 & 5 & 30 & $5-30$ \\
\hline \multicolumn{7}{|l|}{ Dependent Variables } \\
\hline Positive Cognitive Processing (CPO & 166 & 14.71 & 2.92 & 1.83 & 18 & $0-18$ \\
\hline Negative Cognitive Processing (CPC & 167 & 2.74 & 2.27 & 0 & 11.75 & $0-12$ \\
\hline Posttraumatic Growth (PTGI, Total) & 165 & 48.84 & 27.08 & 0 & 105 & $0-105$ \\
\hline
\end{tabular}

Table 6 provides the correlation coefficients for the demographic, independent, and dependent variables utilized in the study.

Table 6

\section{Correlation Coefficients}

\begin{tabular}{|c|c|c|c|c|c|c|c|c|c|c|c|}
\hline Variable & 1 & 2 & 3 & 4 & 5 & 6 & 7 & 8 & 9 & 10 & 11 \\
\hline 1. Age & 1.00 & & & & & \multirow{5}{*}{\multicolumn{6}{|c|}{$\begin{array}{l}\text { Stage of Dx = Stage of Diagnosis } \\
\text { Time = Time Since Treatment Completion } \\
\text { L.O. Threat = Life Outlook Threat } \\
\text { Phys Threat = Physical Threat } \\
\text { Pos Cog Processing = Positive Cognitive Processing } \\
\text { Neg Cog Processing = Negative Cognitive Processing } \\
\text { PTG = Posttraumatic Growth }\end{array}$}} \\
\hline 2. Gender & $.322 * *$ & 1.00 & & & & & & & & & \\
\hline 3. Stage of Dx & .099 & $.211 * *$ & $=1.00$ & & & & & & & & \\
\hline 4. Time & $.167 *$ & $.005-$ & -.136 & 1.00 & & & & & & & \\
\hline 5. L.O. Threat & $-.423 * *$ & $-.182 *-$ & -.065 & -.095 & 1.00 & & & & & & \\
\hline 6. Phys Threat & $-.385 * *$ & -.035 & .075 & -.026 & $.511 * *$ & 1.00 & & & & & \\
\hline 7. Anxiety & $-.284 * *$ & -.127 & .062 & -.001 & $.335 * *$ & $.491 *$ & 1.00 & & & & \\
\hline 8. Depression & -.039 & .087 & $.250 * *$ & -.028 & .153 & $.328 *$ & $.574 * *$ & 1.00 & & & \\
\hline 9. Pos Cog Proc & $.172 *$ & $-.153 *$ & -.081 & .131 & $-.174 *$ & $-.479 * *$ & $-.460 * *$ & $-.516 * *$ & 1.00 & & \\
\hline 10. Neg Cog Proc & c. .132 & .104 & .072 & -.138 & .022 & .069 & $.160 *$ & $.221 * *-$ & $.252 * * 1$ & 1.00 & \\
\hline 11. PTG (Total) - & $-.315 * *$ & $-.236 * *$ & * -.079 & .024 & $.537 * *$ & $.249 * *$ & $.185^{*}$ & .030 & $.204 * *$ & $-.175^{*}$ & 1.00 \\
\hline
\end{tabular}

$* p<.05$ level, two tailed. $* * p<.01$, two tailed 


\section{Mean Comparisons for Variables Between Two Groups}

A One-Way Analysis of Variance (ANOVA) was performed using SPSS 18.0 to compare means of Life Outlook Threat, Physical Threat, Anxiety, Depression, Positive Cognitive Processing, Negative Cognitive Processing, and PTG between survivors who had experienced a recurrence of cancer and those who had not. ANOVAs were conducted instead of independent $t$-tests as Type I error rate (finding a significant result when it does not exist) can increase with multiple $t$ tests. Results indicated that there was a difference in mean scores of the two groups for Physical Threat, $\mathrm{F}(1,164)=7.48, p=$ .007 . More specifically, the non-recurrence group reported lower scores $(M=16.11, S D$ $=6.14)$ compared to the recurrence group $(M=19.95, S D=6.17)$. There was also a difference in means scores of the two groups for Positive Cognitive Processing, with the recurrence group reporting lower scores $(M=13.50, S D=3.53)$ than the non-recurrence group $(M=14.89, S D=2.80), \mathrm{F}(1,164)=4.20, p=.042$.

Given that Positive Cognitive Processing was the dependent variable in Hypothesis 1, and there was a significant difference between scores for those who had experienced a recurrence of cancer and those who had not, a decision was made to take a conservative approach and include Recurrence as a control variable in the regression equation for this hypothesis. However, it is important to note that running an ANOVA comparing samples of 22 and 144 participants may not provide meaningful information. There were no other significant differences.

Specifically related to the variable of Cancer Treatment Received, the database initially had 14 categories, including various combinations of treatments. Yet, many of these categories included very few survivors. Thus, in order to collapse the groups for 
future analyses, and create a variable that would be meaningful in terms of interpretation and implication of the study's results, two groups were created based on a survivor receiving only one type of cancer treatment (i.e., chemotherapy only) or multiple types of cancer treatments (i.e., chemotherapy and radiation). Twenty-nine percent of the sample $(n=49)$ received a single cancer treatment and $71 \%(n=120)$ received multiple cancer treatments. All subsequent analyses utilized this categorized variable of treatment received.

An ANOVA based upon Treatment Received indicated that there was a difference in mean scores between the two groups for Life Outlook Threat, with those who received a single cancer treatment reporting lower scores $(M=9.10, S D=5.01)$ than those who received multiple cancer treatments $(M=10.69, S D=4.48), \mathrm{F}(1,165)=4.08, p=.045$. There was also a difference between the groups for Anxiety, with survivors who received a single cancer treatment reporting lower scores $(M=3.59, S D=4.02)$ compared to those who received multiple treatments $(M=5.19, S D=3.67), \mathrm{F}(1,165)=6.19, p=.014$. Furthermore, results indicated a significant difference between the two groups for PTG, with survivors who received a single cancer treatment reporting lower scores $(M=36.81$, $S D=28.82)$ than those who received multiple treatments $(M=53.77, S D=24.82), \mathrm{F}(1$, $163)=14.44, p<.001$. Thus, for the hypotheses where PTG was the dependent variable (Hypotheses 2 and 4), Treatment Received was included as a control variable in the regression equations. No other significant results were found.

Another ANOVA was performed to compare means of Life Outlook Threat, Physical Threat, Anxiety, Depression, Positive Cognitive Processing, Negative Cognitive Processing, and PTG between survivors who had experienced permanent after-effects of 
treatment and those who had not. Results indicated there was a difference in mean scores between the two groups for Life Outlook Threat, with those without permanent aftereffects of treatment reporting lower scores $(M=8.69, S D=4.86)$ than those with permanent after-affects $(M=11.52, S D=4.17), \mathrm{F}(1,162)=16.07, p=.001$. There was also a significant difference between the groups for Physical Threat, with survivors who have not experienced permanent after-effects of treatment reporting lower scores $(M=$ $15.28, S D=6.28)$ compared to survivors who had $(M=17.97, S D=6.01), \mathrm{F}(1,161)=$ $7.79, p=.006)$. For Anxiety, results revealed that those without permanent after-effects $(M=3.76, S D=3.40)$ had significantly lower scores than those with them $(M=5.58, S D$ $=4.03), \mathrm{F}(1,162)=9.57, p=.002)$. A similar difference was found related to Depression, $\mathrm{F}(1,162)=8.82, p=.003)$, with those without after-effects reporting lower scores $(M=2.11, S D=2.54)$ compared to survivors with after-effects $(M=3.56, S D=$ 3.55). Finally, results indicated there was a difference between the two groups for Positive Cognitive Processing with those who had experienced permanent after-effects of treatment reporting lower scores $(M=13.84, S D=3.05)$ that those who had no aftereffects $(M=15.74, S D=2.44), \mathrm{F}(1,161)=18.65, p=.001)$. Given this result, a decision was made to include permanent After-Effects of Treatment in the regression equation for Hypothesis 1 since Positive Cognitive Processing was the dependent variable for that hypothesis. No other significant differences were found.

\section{Mean Comparisons for Variables Between Three or More Groups}

An Analysis of Variance (ANOVA) was conducted to compare means of Life Outlook Threat, Physical Threat, Anxiety, Depression, Positive Cognitive Processing, Negative Cognitive Processing, and PTG among breast, prostate and colorectal cancer 
survivors. Results revealed that for Life Outlook Threat, Cancer Type was significant, F $(2,164)=3.23, p=.042$ for the breast and prostate cancer groups. A Tukey posthoc comparison indicated that prostate cancer survivors reported lower scores $(M=8.33, S D$ $=4.98)$ compared to breast cancer survivors $(M=10.99, S D=4.56)$. Cancer Type was also significant for the variable of Negative Cognitive Processing, F $(2,164)=5.28, p=$ .006. A Tukey posthoc comparison revealed that breast cancer survivors reported lower scores $(M=2.18, S D=2.31)$ than colorectal cancer survivors $(M=3.36, S D=2.09)$.

Furthermore, the results indicated that for PTG, Cancer Type was significant, F $(2,162)=10.74, p<.001$. The posthoc comparison revealed a statistically significant difference between breast $(M=55.45, S D=26.30)$ and prostate cancer $(M=27.52, S D=$ 25.20) survivors as well as prostate and colorectal cancer $(M=47.97, S D=24.78)$ survivors. Prostate cancer survivors reported lower PTG scores compared to both breast and colorectal cancer survivors. Thus, for the hypotheses where Negative Cognitive Processing or PTG were the dependent variables (Hypotheses 2, 3 and 4) Cancer Type will be included in the regression analysis in order to control for the influence of this variable. There were no other statistically significant results.

An ANOVA was also performed to compare mean scores of survivors from different locations for cancer treatment on the variables described above. It is important to note that the database initially included 67 different treatment locations, counting various combinations of locations. Yet, the majority of these locations (54) included only one survivor. Thus, treatment location was categorized based upon percentage of participants (greater than 5\% of sample) going to a particular site. Four groups emerged: Rocky Mountain Cancer Centers (RMCC) Rose Office (20.7\%) RMCC Midtown Office 
(14.8\%), RMCC Skyridge Office (8.9\%) and All Other Locations (50.3\%). All subsequent analyses utilized the categorized variable of location of treatment. The ANOVA revealed that location of treatment was significant for Negative Cognitive Processing, F $(3,154)=4.75, p=.003$. The Tukey posthoc comparison indicated that survivors treated at the RMCC Rose Office had lower scores $(M=1.65, S D=1.54)$ than survivors treated at All Other Locations $(M=3.19, S D=2.41)$. Therefore, Location of Treatment was included in the regression equation where Negative Cognitive Processing was the dependent variable (Hypothesis 3). There were no other statistically significant results.

One additional ANOVA was then conducted to compare means on Life Outlook Threat, Physical Threat, Anxiety, Depression, Positive Cognitive Processing, Negative Cognitive Processing, and PTG among four groups based on the degree that permanent after-effects interfere with a survivor's daily life. This analysis was performed in order to determine if Interference of After-Effects of Treatment needed to be included in the regression equations for the study's main hypotheses. The ANOVA indicated that there was a significant difference for Physical Threat, $\mathrm{F}(3,28)=2.67, p=.043$. A Tukey posthoc comparison showed that those with "a little" interference $(M=16.71, S D=5.94)$ reported lower scores than those who had experienced "a lot" of interference $(M=22.67$, $S D=5.20)$. On the Depression score, there was a difference, $\mathrm{F}(3,81)=4.51, p=.003$, between the "none" group $(M=1.58, S D=1.61)$ and "a lot" group $(M=6.44, S D=$ 5.57), with the "none" group reporting lower scores. There was also a significant difference, $\mathrm{F}(3,83)=3.16, p=.023$, between the "none" group $(M=14.83, S D=2.03)$ and "a lot" group $(M=11.34, S D=4.48)$ for Positive Cognitive Processing, with the "a 
lot" group reporting lower scores. For Negative Cognitive Processing, there was a significant difference, $\mathrm{F}(3,83)=3.33, p=.023$, between the "none" group $(M=1.86$, $S D=1.67)$ and the "a little" group $(M=3.72, S D=2.67)$, with the "none" group reporting lower scores. Therefore, for the hypotheses where Positive and Negative Cognitive Processing were the dependent variables (Hypotheses 1 and 3), Interference of After-Effects of Treatment was included as a control variable in the regression equations.

\section{Primary Analyses}

This section first addresses the assumptions of multiple regression. Second, identification and treatment of outliers is discussed. Third, the analyses and results for each of the four research hypotheses are presented. The alpha level was set at $p<.05$ for all statistical analyses.

The multiple regression assumptions of normality, linearity, homoscedasticity of residuals, mean independence and absence of multicollinearity and outliers (Tabachnick \& Fidell, 2007) were examined and evaluated as follows. Normality was assessed by plotting the residuals of each regression model using histograms that were overlaid with a normal curve. A visual inspection of the histograms indicated that the residuals for the models reasonably followed a normal distribution and it was determined that the assumption of normality was met (Tabachnick \& Fidell, 2007).

Linearity was assessed using the Normal Probability Plot (P-P) of the Regression Standardized Residual. The Normal P-P Plot for all models revealed a reasonably straight line from bottom left to top right indicating a straight-line relationship between variables as well as no major deviations from normality. Homoscedasticity and independence of residuals was visually examined using a scatterplot of the standardized residuals. The 
residuals were roughly rectangularly distributed, as opposed to curvilinear or cone shaped, with scores relatively concentrated in the center, along the zero point line, and evenly distributed on both sides of the centerline, indicating that these assumptions were met (Tabachnick \& Fidell, 2007).

Mean independence is an assumption that addresses error term. In assessing independence, there are a few factors that need to be considered. The first factor is that independent variables relevant to the analyses are included in the regression model. Mean independence is violated when independent variables that influence the outcome variables are omitted from the regression model (Freedman, 2005). Thus, the variables used in the models were determined by existing literature and existing theories in order to determine that the appropriate predictors and control variables were selected. A second factor is that the variables are, ideally, measured without error. Therefore, the measures used in this study were selected based on their reliability. Each of the independent and dependent measures produced average to above average reliability within the current sample. To further evaluate independence of errors, Durbin-Watson coefficient $d$ values were examined for each regression model. Values lower than two indicate autocorrelation (Garson, 2010). The first regression equation had a coefficient $d$ value of 2.08, which demonstrated that there were no autocorrelations and there was an independence of errors. While, the second, third, and fourth models had Durbin-Watson values of 1.83, 1.90, and 1.82 respectively, these values are only slightly less than two and therefore there was no strong evidence for autocorrelation.

Multicollinearity was assessed using tolerance and Variance Inflation Factor (VIF) values. Tolerance is an indicator of how much variability of the specified independent 
variable is not explained by the other independent variables in the model. The VIF is the inverse of the tolerance value (Pallant, 2007). The cutoff values for tolerance and VIF used in the analyses were based on Pallant's (2007) cutoff levels of less than .10 for tolerance and above 10 for VIF. Based on these values, the variables in all of the models did not indicate the presence of multicollinearity.

As noted in the preliminary analyses, correlations coefficients were also examined to detect strong correlations between variables. Moderate correlations were found between Life Outlook Threat and Physical Threat $(r=.511, p<.01)$ as well as Anxiety and Depression $(r=.574, p<.01)$. Therefore a decision was made to separate each of these variable pairs, while keeping all other variables in the model the same, and run four follow-up regressions for each hypothesis: one with Life Outlook Threat and without Physical Threat, one with Physical Threat and without Life Outlook, one with Anxiety without Depression, and one with Depression without Anxiety. This was done in order to examine the individual impact of the four variables in each model.

Outliers were detected by inspecting the Mahalanobis distances that were found in the results. To identify which cases were outliers, the critical chi-square values were determined for each regression equation using the number of independent variables as the degrees of freedom and subsequently looking up the value in Tabachnick and Fidell's Multivariate Statistics text (2007, Table C.4). The authors suggest using an alpha level of .001 when determining critical chi-square values. Using these criteria, the maximum Mahalanobis values for the first three models indicated that there were no values in the dataset that exceeded the critical value, suggesting that no outliers were present. For the fourth model, the maximum Mahalanobis Distance value exceeded the critical value, 
suggesting that one or more outliers were present. To determine which cases were outliers, the database was sorted according to Mahalanobis Distance, which revealed that there was one outlier in the database. While it is unlikely that one outlier would make a substantial difference in the results, a conservative approach was taken and the equation for Hypothesis 4 was re-run without the outlier. It is important to keep in mind that once the single outlier was removed from the database the sample size decreased by one as sample size influences the values obtained in the results.

Statistical Analyses Addressing Research Hypotheses

Hypothesis 1: The first hypothesis stated, "Anxiety, Depression, and Perceived Threat will predict Positive Cognitive Processing over and beyond the contribution of the demographic control variables. It is hypothesized that Anxiety and Depression will negatively predict Positive Cognitive Processing, while Perceived Threat will positively predict Positive Cognitive Processing."

To address this hypothesis, a hierarchical regression was utilized to investigate the relative contributions of Anxiety, Depression, and Perceived Threat in the prediction of Positive Cognitive Processing after controlling for the demographic variables of Age, Gender, Stage of Diagnosis, Time Since Treatment Completion, Recurrence, AfterEffects of Treatment, and Interference of After-Effects. The last three variables were added to the regression model after the preliminary analyses revealed that there were differences for these variables related to the dependent variable of Positive Cognitive Processing. The demographic variables were entered in the first block, Anxiety and Depression were entered in the second block, and Life Outlook Threat and Physical Threat were entered in the third block. 
The demographic control variables in the first equation (Block 1), significantly contributed to the model, $\mathrm{R}^{2}=.226, \mathrm{~F}(7,76)=3.17, p<.01$, accounting for $22.6 \%$ of the variance. Anxiety and Depression (Block 2) also contributed to the model. After these variables were entered the total variance explained by the model as a whole was $41.1 \%, \mathrm{~F}$ $(9,74)=5.74, p<.001$. Anxiety and Depression explained an additional $18.5 \%$ of the variance in Positive Cognitive Processing, after controlling for the demographic variables, $\Delta \mathrm{R}^{2}=.185, \Delta \mathrm{F}(2,74)=11.62, p<.001$. Life Outlook Threat and Physical Threat (Block 3) contributed to the model as well. When these variables were included, the total variance explained by the model as a whole was $46.9 \%, \mathrm{~F}(11,72)=5.77, p<$ .001. Life Outlook Threat and Physical Threat explained an additional 5.8\% of the variance in Positive Cognitive Processing, after controlling for the demographic variables as well as Anxiety and Depression, $\Delta \mathrm{R}^{2}=.058, \Delta \mathrm{F}(2,72)=3.91, p<.05$. In the final model, the variables of Physical Threat, Depression, and After-Effects of Treatment were statistically significant, with Physical Threat recording the highest beta value $(\beta=-.32, p$ $=.007)$ compared to Depression $(\beta=-.27, p=.025)$ and After-Effects of Treatment $(\beta=-$ $.19, p=.045)$. These findings suggest that a decrease in physical threat and depression as well as not having experienced permanent after-effects of treatment appear to be related to an increase in positive cognitive processing. Table 7 provides a summary of the statistical findings. 


\section{Table 7}

Hierarchical Regression of Age, Gender, Stage of Diagnosis, Time Since Treatment Completion, Recurrence, and Interference of After-Effects on Positive Cognitive Processing $(n=166)$

\begin{tabular}{|c|c|c|c|}
\hline \multirow{3}{*}{$\begin{array}{l}\text { Variable } \\
\text { Block } 1 .\end{array}$} & \multicolumn{3}{|c|}{ Positive Cognitive Processing } \\
\hline & $B$ & $S E B$ & $\beta$ \\
\hline & & & \\
\hline Age & .028 & .029 & .108 \\
\hline Gender & -.761 & .749 & -.121 \\
\hline Stage of Diagnosis & .036 & .267 & .014 \\
\hline Time Since Treatment Completion & .031 & .133 & .025 \\
\hline Recurrence & -.609 & .973 & -.070 \\
\hline After-Effects of Treatment & -1.73 & .624 & $-.296 * *$ \\
\hline Interference of After-Effects & -.822 & .333 & $-.267 *$ \\
\hline $\mathrm{R}^{2}$ & & .226 & \\
\hline $\mathrm{F}$ for change in $\mathrm{R}^{2}$ & & $3.17 * *$ & \\
\hline \multicolumn{4}{|l|}{ Block 2.} \\
\hline Age & .016 & .027 & .061 \\
\hline Gender & -.935 & .667 & -.148 \\
\hline Stage of Diagnosis & .216 & .240 & .086 \\
\hline Time Since Treatment Completion & .090 & .118 & .072 \\
\hline Recurrence & -.542 & .861 & -.063 \\
\hline After-Effects of Treatment & -1.10 & .568 & -.188 \\
\hline Interference of After-Effects & -.460 & .313 & -.149 \\
\hline Anxiety & -.188 & .089 & $-.247 *$ \\
\hline Depression & -.260 & .112 & $-.284 *$ \\
\hline $\mathrm{R}^{2}$ & & .411 & \\
\hline $\mathrm{F}$ for change in $\mathrm{R}^{2}$ & & 11.62 & \\
\hline
\end{tabular}


Variable

\begin{tabular}{lccc}
\hline & $B$ & $S E B$ & $\beta$ \\
Block 3. & .003 & .027 & .013 \\
Age & -.868 & .644 & -.138 \\
Gender & .267 & .232 & .107 \\
Stage of Diagnosis & .106 & .114 & .085 \\
Time Since Treatment Completion & -.033 & .850 & -.004 \\
Recurrence & -1.13 & .556 & $-.194 *$ \\
After-Effects of Treatment & -.374 & .303 & -.121 \\
Interference of After-Effects & -.122 & .091 & -.160 \\
Anxiety & -.248 & .108 & $-.270^{*}$ \\
Depression & .091 & .067 & .147 \\
Life Outlook Threat & -.151 & .055 & $-.324 * *$ \\
Physical Threat & & .469 & \\
$\mathrm{R}^{2}$ & & $3.91 *$ & \\
F for change in $\mathrm{R}^{2}$ & & & \\
\hline
\end{tabular}

Note. Positive Cognitive Processing: $\mathrm{R}^{2}=.226 ; \Delta \mathrm{R}^{2}=.185$ for Block $2(p<.001) ; \Delta \mathrm{R}^{2}=.058$ for Block 3 $(p<.05) ; * p<.05 * * p<.01$

Follow-Up Analyses for Hypothesis 1

Given the high correlation between Anxiety and Depression $(r=.574, p<.01)$, a follow-up regression was conducted for Hypothesis 1 with Anxiety only in Block 2. In contrast to what was found in the primary analysis for Hypothesis 1, results revealed that Anxiety was a significant predictor in the model, $\mathrm{R}^{2}=.368, \mathrm{~F}(8,75)=5.46, p<.001$. Anxiety explained an additional $14.2 \%$ of the variance in Positive Cognitive Processing, after controlling for the demographic variables, $\Delta \mathrm{R}^{2}=.142, \Delta \mathrm{F}(1,75)=16.87, p<.001$, with a $\beta$ of -.308 $(p=.004)$ in the final model. This finding suggests that Anxiety alone is a significant predictor of Positive Cognitive Processing such that a decrease in anxiety is 
related to an increase in positive cognitive processing. However, Anxiety shares enough variance with Depression that when Depression is entered into the equation, the effect of Anxiety "washes out" and is no longer significant. An additional regression was performed with Depression only in the second block. Results revealed that, as expected from previous analysis, Depression contributed to the model, $\mathrm{R}^{2}=.376, \mathrm{~F}(8,75)=5.64$, $p<.001$. Depression explained an additional $15 \%$ of the variance in Positive Cognitive Processing, after controlling for the demographic variables, $\Delta \mathrm{R}^{2}=.150, \Delta \mathrm{F}(1,75)=$ $17.99, p=.001$, with a $\beta$ value of $-.354(p=.001)$. This finding indicates that a decrease in depression is associated with an increase in positive cognitive processing. Thus, one variable could be substituted for the other or Anxiety and Depression could be combined to form a single variable.

Life Outlook Threat and Physical Threat are also highly correlated $(r=.511, p<$ .01). Therefore, a regression was performed with Life Outlook Threat only in Block 3. Results revealed that Block 3 did not contribute significantly to the model, $\Delta \mathrm{R}^{2}=.001$, $\Delta \mathrm{F}(1,73)=.135, p=.716$, accounting for only $0.1 \%$ of the variance. When the regression was conducted again with Physical Threat only in Block 3, the results indicated that Physical Threat significantly contributed to the model, explaining an additional $4.4 \%$ of the variance in Positive Cognitive Processing, after controlling for the demographic variables as well as Anxiety and Depression, $\Delta \mathrm{R}^{2}=.044, \Delta \mathrm{F}(1,73)=5.89$, $p<.05$. In the final model, Physical Threat had a $\beta$ value of $-2.66(p=.018)$. This suggests that a decrease in physical threat is related to an increase in positive cognitive processing. Therefore, it appears that Life Outlook Threat and Physical Threat have a unique and separate relationship to Positive Cognitive Processing. 
Hypothesis 2. The second hypothesis stated, "Positive Cognitive Processing will significantly predict PTG over and beyond the demographic control variables, Anxiety, Depression, and Perceived Threat. In other words, Positive Cognitive Processing will account for a significant amount of the variance of PTG beyond the demographic control variables (i.e., Age, Gender, Stage of Diagnosis, Time Since Treatment Completion), Anxiety, Depression, and Perceived Threat. It is hypothesized that Positive Cognitive Processing and Perceived Threat will positively predict PTG, while Anxiety and Depression will negatively predict PTG."

To address this hypothesis, a hierarchical regression was utilized to investigate the relative contributions of Anxiety, Depression, Perceived Threat, and Positive Cognitive Processing in the prediction of PTG after controlling for the demographic variables of Age, Gender, Stage of Diagnosis, Time Since Treatment Completion, Cancer Type and Treatment Received. The last two variables were added to the regression model after the preliminary analyses revealed that there were differences for these variables related to the dependent variable of PTG. The demographic variables were entered in the first block, Anxiety and Depression were entered in the second block, Life Outlook Threat and Physical Threat were entered in the third block, and Positive Cognitive Processing was entered in the fourth block.

The demographic control variables in the second equation (Block 1), did significantly contribute to the model, $\mathrm{R}^{2}=.161, \mathrm{~F}(6,150)=4.81, p<.001$, accounting for $16.1 \%$ of the variance. Anxiety and Depression (Block 2) did not contribute to the model. After these variables were entered, the total variance explained by the model as a whole was $16.7 \%, \mathrm{~F}(8,148)=3.71, p=.001$. Anxiety and Depression explained only an 
additional $0.6 \%$ of the variance in PTG, after controlling for the demographic variables, $\Delta \mathrm{R}^{2}=.006, \Delta \mathrm{F}(2,148)=.499, p=.608$. Life Outlook Threat and Physical Threat (Block 3) significantly contributed to the model. After these variables were entered, the total variance explained by the model as a whole was $34.9 \%, \mathrm{~F}(10,146)=7.82, p<.001$. These variables explained an additional $18.2 \%$ of the variance in PTG, after controlling for the demographic variables as well as Anxiety and Depression, $\Delta \mathrm{R}^{2}=.182, \Delta \mathrm{F}(2$, 146) $=20.39, p<.001$. Positive Cognitive Processing (Block 4) also contributed to the model. When this variable was included, the total variance explained by the model as a whole was 44.5\%, F $(11,145)=10.59, p<.001$. Positive Cognitive Processing explained an additional $9.7 \%$ of the variance in PTG, after controlling for the demographic variables, Anxiety, Depression, Life Outlook Threat and Physical Threat, $\Delta \mathrm{R}^{2}=.097, \Delta \mathrm{F}$ $(1,145)=25.25, p<.001$.

In the final model, the variables of Life Outlook Threat, Positive Cognitive Processing, and Treatment Received were statistically significant, with Life Outlook Threat recording the highest beta value $(\beta=.452, p<.001)$ followed by Positive Cognitive Processing $(\beta=.416, p<.001)$, and Treatment Received $(\beta=.142, p<.05)$. These findings suggest that an increase in both life outlook threat and positive cognitive processing appear to be related to an increase in posttraumatic growth. Furthermore, having received more than one cancer treatment as compared to a single treatment, appears to be related to an increase in PTG. Table 8 provides a summary of the results. 
Table 8

Hierarchical Regression of Age, Gender, Stage of Diagnosis, Time Since Treatment Completion, Cancer Type Treatment Received, Life Outlook Threat, Physical Threat, Anxiety, Depression, and Positive Cognitive Processing on Posttraumatic Growth ( $n=$ 165)

Variable Posttraumatic Growth

(2)

Block 1.

$B \quad S E B \quad \beta$

Age

$-.589 \quad .195 \quad-.247 * *$

Gender

$\begin{array}{lll}-6.97 & 5.23 & -.119\end{array}$

Stage of Diagnosis

$\begin{array}{lll}-1.11 & 1.94 & -.048\end{array}$

Time Since Treatment Completion .667

.906

.058

Cancer Type

1.88

2.84

.064

Treatment Received

12.44

4.83

$.209 *$

$\mathrm{R}^{2}$

.161

$\mathrm{F}$ for change in $\mathrm{R}^{2}$

$4.81 * *$

Block 2.

$\begin{array}{lccc}\text { Age } & -.533 & .204 & -.22 *^{*} \\ \text { Gender } & -6.49 & 5.29 & -.111 \\ \text { Stage of Diagnosis } & -1.09 & 2.00 & -.047 \\ \text { Time Since Treatment Completion } & .606 & .911 & .052 \\ \text { Cancer Type } & 1.71 & 2.83 & .058 \\ \text { Treatment Received } & 11.95 & 4.89 & .201^{*} \\ \text { Anxiety } & .675 & .694 & .095 \\ \text { Depression } & -.314 & .824 & -.037 \\ \mathrm{R}^{2} & & .167 & \\ \text { F for change in } \mathrm{R}^{2} & & .499 & \end{array}$




\begin{tabular}{|c|c|c|c|}
\hline & $B$ & $S E B$ & $\beta$ \\
\hline \multicolumn{4}{|l|}{ Block 3.} \\
\hline Age & -.160 & .196 & -.067 \\
\hline Gender & -5.26 & 4.75 & -.090 \\
\hline Stage of Diagnosis & -.375 & 1.79 & -.016 \\
\hline Time Since Treatment Completion & .874 & .813 & .076 \\
\hline Cancer Type & 1.38 & 2.55 & .047 \\
\hline Treatment Received & 10.94 & 4.38 & $.184 *$ \\
\hline Anxiety & -.040 & .655 & -.006 \\
\hline Depression & -.401 & .736 & -.047 \\
\hline Life Outlook Threat & 2.86 & .477 & $.495 * *$ \\
\hline Physical Threat & -.097 & .382 & -.022 \\
\hline $\mathrm{R}^{2}$ & & .349 & \\
\hline $\mathrm{F}$ for change in $\mathrm{R}^{2}$ & & $20.39 * *$ & \\
\hline \multicolumn{4}{|l|}{ Block 4.} \\
\hline Age & -.230 & .182 & -.097 \\
\hline Gender & -1.11 & 4.48 & -.019 \\
\hline Stage of Diagnosis & -1.06 & 1.67 & -.046 \\
\hline Time Since Treatment Completion & .280 & .762 & .024 \\
\hline Cancer Type & .783 & 2.36 & .027 \\
\hline Treatment Received & 8.48 & 4.09 & $.142 *$ \\
\hline Anxiety & .429 & .613 & .061 \\
\hline Depression & .822 & .724 & .096 \\
\hline Life Outlook Threat & 2.62 & .444 & $.452 * *$ \\
\hline Physical Threat & .489 & .373 & .113 \\
\hline Positive Cognitive Processing & 3.86 & .767 & $.416^{* *}$ \\
\hline $\mathrm{R}^{2}$ & & .445 & \\
\hline $\mathrm{F}$ for change in $\mathrm{R}^{2}$ & & $25.25 * *$ & \\
\hline
\end{tabular}

Note. Posttraumatic Growth: $\mathrm{R}^{2}=.161 ; \Delta \mathrm{R}^{2}=.006$ for Block $2(p=.608) ; \Delta \mathrm{R}^{2}=.182$ for Block $3(p<$ $.001) ; \Delta \mathrm{R}^{2}=.097$ for Block $4(p<.001) ; * p<.05 * * p<.01$ 


\section{Follow-Up Analyses for Hypothesis 2}

Due to the high correlation between Life Outlook Threat and Physical Threat $(r=$ $.511, p<.01)$, and given the significance of these variables in the main regression, a follow-up regression was conducted for Hypothesis 2 with Life Outlook Threat only in Block 3. Results revealed that Block 3 did contribute significantly to the model, $\mathrm{R}^{2}=$ $.349, \mathrm{~F}(9,147)=8.74, p<.001$, explaining an additional $18.2 \%$ of the variance in PTG, after controlling for the demographic variables as well as Anxiety and Depression, $\Delta \mathrm{R}^{2}=$ $.182, \Delta \mathrm{F}(1,147)=40.98, p<.001$. When the regression was conducted again with Physical Threat only in Block 3, the results indicated that while the model as a whole was significant at Block $3, \mathrm{R}^{2}=.188, \mathrm{~F}(9,147)=3.78, p<.001$, Block 3 did not individually contribute to the model. Physical Threat explained only an additional $2.1 \%$ of the variance in PTG, after controlling for the demographic variables as well as Anxiety and Depression, $\Delta \mathrm{R}^{2}=.021, \Delta \mathrm{F}(1,147)=3.77, p=.054$. Yet, in the final model, Physical Threat had a significant $\beta$ value of $.310(p=.001)$. This suggests that although Physical Threat did not significantly change the variance explained by the model as a whole, when it is entered into the model alone it is a significant individual predictor of PTG such that an increase in physical threat is related to an increase in PTG. However, when Life Outlook Threat is entered along with Physical Threat, the influence of Physical Threat is overshadowed by the variance it shares with Life Outlook Threat and is no longer significant.

Hypothesis 3. The third hypothesis stated, "Anxiety, Depression, and Perceived Threat will predict Negative Cognitive Processing over and beyond the contribution of 
the demographic control variables. It is hypothesized that Anxiety, Depression, and Perceived Threat will all positively predict Negative Cognitive Processing."

To investigate this hypothesis, hierarchical regression was utilized to investigate the relative contributions of Anxiety, Depression, and Perceived Threat in the prediction of Negative Cognitive Processing after controlling for the demographic variables of Age, Gender, Stage of Diagnosis, Time Since Treatment Completion, Cancer Type, Location of Treatment, and Interference of After-Effects of Treatment. The last three variables were added to the regression model after the preliminary analyses revealed that there were differences for these variables related to the dependent variable of Negative Cognitive Processing. The demographic variables were entered in the first block, Anxiety and Depression were entered in the second block, and Life Outlook Threat and Physical Threat were entered in the third block.

The demographic control variables in the third equation (Block 1), did not significantly contribute to the model, $\mathrm{R}^{2}=.121, \mathrm{~F}(7,75)=1.48, p=.187$, accounting for $12 \%$ of the variance. Anxiety and Depression (Block 2) also did not contribute to the model. After these variables were entered the total variance explained by the model as a whole was $18.4 \%, \mathrm{~F}(9,73)=1.83, p=.078$. Anxiety and Depression explained an additional $6.2 \%$ of the variance in Negative Cognitive Processing, after controlling for the demographic variables, $\Delta \mathrm{R}^{2}=.062, \Delta \mathrm{F}(2,73)=2.79, p=.068$. Life Outlook Threat and Physical Threat (Block 3) did not contribute to the model as well. When these variables were included, the total variance explained by the model as a whole was $18.6 \%$, $\mathrm{F}(11,71)=1.48, p=.159$. Life Outlook Threat and Physical Threat explained only an additional $0.3 \%$ of the variance in Negative Cognitive Processing, after controlling for 
the demographic variables as well as Anxiety and Depression, $\Delta \mathrm{R}^{2}=.003, \Delta \mathrm{F}(2,71)=$ $.111, p=.859$. In the final model, none of the variables included in the model were statistically significant. These findings suggest that none of the included variables are related to negative cognitive processing. Table 9 provides a summary of the statistical findings.

Table 9

Hierarchical Regression of Age, Gender, Stage of Diagnosis, Time Since Treatment Completion, Cancer Type, Location of Treatment and Interference of After-Effects on Negative Cognitive Processing $(n=167)$

\begin{tabular}{|c|c|c|c|}
\hline \multirow{3}{*}{$\begin{array}{l}\text { Variable } \\
\text { Block } 1 .\end{array}$} & \multicolumn{3}{|c|}{ Negative Cognitive Processing } \\
\hline & $B$ & $S E B$ & $\beta$ \\
\hline & & & \\
\hline Age & .018 & .024 & .089 \\
\hline Gender & -.276 & .636 & -.056 \\
\hline Stage of Diagnosis & -.074 & .237 & -.038 \\
\hline Time Since Treatment Completion & -.129 & .110 & -.133 \\
\hline Cancer Type & .652 & .341 & .264 \\
\hline Location of Treatment & .399 & .213 & .207 \\
\hline Interference of After-Effects & -.023 & .271 & -.009 \\
\hline $\mathrm{R}^{2}$ & & .121 & \\
\hline $\mathrm{F}$ for change in $\mathrm{R}^{2}$ & & 1.48 & \\
\hline \multicolumn{4}{|l|}{ Block 2.} \\
\hline Age & .026 & .024 & .130 \\
\hline Gender & -.244 & .625 & -.050 \\
\hline Stage of Diagnosis & -.159 & .236 & -.082 \\
\hline Time Since Treatment Completion & -.146 & .108 & -.150 \\
\hline Cancer Type & .636 & .333 & .258 \\
\hline Location of Treatment & .400 & .208 & .208 \\
\hline Interference of After-Effects & -.174 & .279 & -.073 \\
\hline
\end{tabular}




\begin{tabular}{lccc}
\hline Variable & \multicolumn{3}{c}{ Negative Cognitive Processing } \\
\hline Anxiety & $B$ & $S E B$ & $\beta$ \\
Depression & .074 & .082 & .125 \\
$\mathrm{R}^{2}$ & & .124 & .174 \\
$\mathrm{~F}$ for change in $\mathrm{R}^{2}$ & & .184 & \\
Block 3. & & 2.79 & \\
Age & .030 & .026 & .151 \\
Gender & -.241 & .637 & -.049 \\
Stage of Diagnosis & -.154 & .240 & -.079 \\
Time Since Treatment Completion & -.144 & .109 & -.149 \\
Cancer Type & .637 & .337 & .258 \\
Location of Treatment & .406 & .214 & .211 \\
Interference of After-Effects & -.184 & .286 & -.077 \\
Anxiety & .064 & .089 & .108 \\
Depression & .123 & .103 & .173 \\
Life Outlook Threat & .025 & .064 & .151 \\
Physical Threat & .005 & .052 & .015 \\
$\mathrm{R}^{2}$ & & .186 & \\
F for change in R & & .111 & \\
& & & \\
\hline
\end{tabular}

Note. Negative Cognitive Processing: $\mathrm{R}^{2}=.121 ; \Delta \mathrm{R}^{2}=.062$ for Block $2(p=.068) ; \Delta \mathrm{R}^{2}=.003$ for Block 3 $(p=.895)$

\section{Follow-Up Analyses for Hypothesis 3}

Given that no statistically significant results were found in the primary regression equation for Hypothesis 3, no follow-up analyses were performed.

Hypothesis 4. The fourth hypothesis stated, "Negative Cognitive Processing will significantly predict PTG over and beyond the demographic control variables, Anxiety, Depression, and Perceived Threat. In other words, Negative Cognitive Processing will 
account for a significant amount of the variance of PTG beyond the demographic control variables (i.e., Age, Gender, Stage of Diagnosis, Time Since Treatment Completion), Anxiety, Depression, and Perceived Threat. It is hypothesized that Negative Cognitive Processing, Anxiety, and Depression will negatively predict PTG, while Perceived Threat will positively predict PTG."

To address this hypothesis, hierarchical regression was utilized to investigate the relative contributions of Anxiety, Depression, Perceived Threat, and Negative Cognitive Processing in the prediction of PTG after controlling for the demographic variables of Age, Gender, Stage of Diagnosis, Time Since Treatment Completion, Cancer Type and Treatment Received. The last two variables were added to the regression model after the preliminary analyses revealed that there were differences for these variables related to the dependent variable of PTG. The demographic variables were entered in the first block, Anxiety and Depression were entered in the second block, Life Outlook Threat and Physical Threat were entered in the third block, and Negative Cognitive Processing was entered in the fourth block.

The demographic control variables in the fourth equation (Block 1) significantly contributed to the model, $\mathrm{R}^{2}=.165, \mathrm{~F}(6,149)=4.91, p<.001$, accounting for $16.5 \%$ of the variance. Anxiety and Depression (Block 2) did not contribute to the model. After these variables were entered, the total variance explained by the model as a whole was $17.1 \%, \mathrm{~F}(8,147)=3.78, p<.001$. Anxiety and Depression explained only an additional $0.6 \%$ of the variance in PTG, after controlling for the demographic variables, $\Delta \mathrm{R}^{2}=$ $.006, \Delta \mathrm{F}(2,147)=.497, p=.609$. Life Outlook Threat and Physical Threat (Block 3) contributed to the model. After these variables were entered the total variance explained 
by the model as a whole was $35.4 \%, \mathrm{~F}(10,145)=7.95, p<.001$. Life Outlook Threat and Physical Threat explained an additional $18.4 \%$ of the variance in PTG, after controlling for the demographic variables as well as Anxiety and Depression, $\Delta \mathrm{R}^{2}=.184$, $\Delta \mathrm{F}(2,145)=20.60, p<.001$. Negative Cognitive Processing (Block 4) did not contribute to the model. After this variable was entered, the total variance explained by the model as a whole was $36.9 \%, \mathrm{~F}(11,144)=7.64, p<.001$. Negative Cognitive Processing explained only an additional $1.4 \%$ of the variance in PTG after controlling for the demographic variables, Anxiety, Depression and Perceived Threat, $\Delta \mathrm{R}^{2}=.014, \Delta \mathrm{F}(1$, $144)=3.29, p=.072$. In the final model, the variables of Life Outlook Threat and Treatment Received were statistically significant, with Life Outlook Threat recording the highest beta value $(\beta=.499, p<.001)$ followed by Treatment Received $(\beta=.176, p<$ $.05)$. These findings suggest that an increase in life outlook threat appears to be related to an increase in posttraumatic growth. Furthermore, receiving multiple cancer treatments as compared to a single form of treatment, appears to be related to an increase in PTG.

Table 10 provides a summary of the results.

Table 10

Hierarchical Regression of Age, Gender, Stage of Diagnosis, Time Since Treatment Completion, Cancer Type, Treatment Received, Life Outlook Threat, Physical Threat, Anxiety, Depression, and Negative Cognitive Processing on Posttraumatic Growth $(n=$ 165)

Variable

Posttraumatic Growth

$B \quad S E B \quad \beta$

Block 1.

Age

Gender
$-.591$

$-7.12$
.195

5.23 $\beta$ $-.248 * *$

$-.122$ 


\begin{tabular}{|c|c|c|c|}
\hline \multirow[t]{2}{*}{ Variable } & \multicolumn{3}{|c|}{ Posttraumatic Growth } \\
\hline & $B$ & $S E B$ & $\beta$ \\
\hline Stage of Diagnosis & -1.22 & 1.95 & -.052 \\
\hline Time Since Treatment Completion & .834 & .922 & .071 \\
\hline Cancer Type & 1.91 & 2.84 & .065 \\
\hline Treatment Received & 12.57 & 4.84 & $.211 *$ \\
\hline $\mathrm{R}^{2}$ & & .165 & \\
\hline $\mathrm{F}$ for change in $\mathrm{R}^{2}$ & & $4.91 * *$ & \\
\hline \multicolumn{4}{|l|}{ Block 2.} \\
\hline Age & -.536 & .204 & $-.225^{* *}$ \\
\hline Gender & -6.62 & 5.30 & -.113 \\
\hline Stage of Diagnosis & -1.17 & 2.00 & -.050 \\
\hline Time Since Treatment Completion & .772 & .927 & .065 \\
\hline Cancer Type & 1.74 & 2.85 & .059 \\
\hline Treatment Received & 12.11 & 4.89 & $.203 *$ \\
\hline Anxiety & .681 & .695 & .096 \\
\hline Depression & -.355 & .825 & -.042 \\
\hline $\mathrm{R}^{2}$ & & .171 & \\
\hline$F$ for change in $R^{2}$ & & .497 & \\
\hline \multicolumn{4}{|l|}{ Block 3.} \\
\hline Age & -.158 & .196 & -.066 \\
\hline Gender & -5.43 & 4.75 & -.093 \\
\hline Stage of Diagnosis & -.473 & 1.79 & -.020 \\
\hline Time Since Treatment Completion & 1.06 & .826 & .090 \\
\hline Cancer Type & 1.43 & 2.54 & .048 \\
\hline Treatment Received & 11.14 & 4.38 & $.187 *$ \\
\hline Anxiety & -.048 & .654 & -.007 \\
\hline
\end{tabular}




\begin{tabular}{|c|c|c|c|}
\hline & $B$ & $S E B$ & $\beta$ \\
\hline Depression & -.452 & .736 & -.053 \\
\hline Life Outlook Threat & 2.86 & .476 & $.496 * *$ \\
\hline Physical Threat & -.072 & .382 & -.017 \\
\hline $\mathrm{R}^{2}$ & & .354 & \\
\hline $\mathrm{F}$ for change in $\mathrm{R}^{2}$ & & $20.60 * *$ & \\
\hline \multicolumn{4}{|l|}{ Block 4.} \\
\hline Age & -.101 & .197 & -.042 \\
\hline Gender & -5.72 & 4.71 & -.098 \\
\hline Stage of Diagnosis & -.698 & 1.78 & -.030 \\
\hline Time Since Treatment Completion & .724 & .840 & .061 \\
\hline Cancer Type & 2.28 & 2.57 & .077 \\
\hline Treatment Received & 10.45 & 4.36 & $.176^{*}$ \\
\hline Anxiety & .074 & .652 & .010 \\
\hline Depression & -.234 & .740 & -.027 \\
\hline Life Outlook Threat & 2.88 & .472 & $.499 * *$ \\
\hline Physical Threat & -.067 & .379 & -.015 \\
\hline Negative Cognitive Processing & -1.69 & .931 & -.134 \\
\hline $\mathrm{R}^{2}$ & & .369 & \\
\hline $\mathrm{F}$ for change in $\mathrm{R}^{2}$ & & 3.29 & \\
\hline
\end{tabular}

Note. Posttraumatic Growth: $\mathrm{R}^{2}=.165 ; \Delta \mathrm{R}^{2}=.006$ for Block $2(p=.609) ; \Delta \mathrm{R}^{2}=.184$ for Block $3(p<$ $.001) ; \Delta \mathrm{R}^{2}=.014$ for Block $4(p=.072) ; * p<.05 * * p<.01$

\section{Follow-Up Analyses for Hypothesis 4}

A follow-up regression was conducted for Hypothesis 4 with Life Outlook Threat only in Block 3. Results were the same as reported above, with Life Outlook Threat contributing significantly to the model, $\mathrm{R}^{2}=.354, \mathrm{~F}(9,146)=8.89, p<.001$. Life 
Outlook Threat explained an additional $18.3 \%$ of the variance in PTG, after controlling for the demographic variables as well as Anxiety and Depression, $\Delta \mathrm{R}^{2}=.183, \Delta \mathrm{F}(1$, $146)=41.44, p<.001$. When the regression was conducted again with Physical Threat only in Block 3, the results indicated that Physical Threat significantly contributed to the model, $\mathrm{R}^{2}=.193, \mathrm{~F}(9,146)=3.88, p<.001$. Physical Threat explained an additional $2.2 \%$ of the variance in PTG, after controlling for the demographic variables as well as Anxiety and Depression, $\Delta \mathrm{R}^{2}=.022, \Delta \mathrm{F}(1,146)=4.01, p=.047$. In the final model, Physical Threat had a $\beta$ value of $.185(p<.05)$. This result suggests that when Physical Threat is entered into the model alone, it is a significant individual predictor of PTG such that an increase in physical threat is related to an increase in PTG. However, when Life Outlook Threat is entered with Physical Threat, the influence of Physical Threat is eclipsed by the variance it shares with Life Outlook Threat and is no longer significant.

\section{Summary}

Chapter Four provided the results of the preliminary analyses of the study followed by the primary analyses, which included the results of the four main regression equations aimed at examining the four research hypotheses as well as follow-up analyses. The results partially supported Hypothesis 1 . In the first and second block of variables, After-Effects of Treatment and Interference of After-Effects of Treatment emerged as significant independent predictors of Positive Cognitive Processing, while Physical Threat emerged as a predictor in the third block. After-Effects of Treatment, Physical Threat and Depression were significant predictors of Positive Cognitive Processing in the final model. Depression had a negative association with Positive Cognitive Processing, which supported the hypothesis. After-Effects of Treatment also had a negative 
relationship with the dependent variable such that experiencing permanent after-effects was related to a decrease in positive cognitive processing. This finding was expected based on the preliminary analysis and makes logical sense as experiencing permanent after-effects may hinder a survivor's ability to engage in positive cognitive processing. However, Physical Threat was negatively associated Positive Cognitive Processing, which is the opposite of what was predicted. Hypothesis 1 was not supported in that Life Outlook Threat and Anxiety were not significant predictors.

Hypothesis 2 was also partially supported. Age and Treatment Received emerged as significant predictors of PTG in the first two blocks of variables. Life Outlook Threat was a predictor in Block 3, along with Treatment Received. In the final model, Life Outlook Threat and Positive Cognitive Processing were positive predictors of PTG, as was Treatment Received. Interestingly, it was found that survivors who received more than one type of cancer treatment reported more PTG than survivors who received a single form of treatment. Hypothesis 2 was not supported in that Physical Threat, Anxiety and Depression were not predictors of PTG. Yet, the follow-up analysis revealed that when Physical Threat was entered into the equation without Life Outlook Threat, it was significant.

Hypothesis 3 was not supported in the study. None of the variables entered in the equation, including the demographic variables, emerged as predictors of Negative Cognitive Processing. Possible reasons for the lack of findings are presented in Chapter Five.

Finally, Hypothesis 4 was partially supported as Age and Treatment Received emerged as significant predictors of PTG in the first two blocks of variables, while Life 
Outlook Threat was significant in the third block, along with Treatment Received. In the final model, Life Outlook Threat and Treatment Received remained significant predictors of PTG. The hypothesis was not supported in that Physical Threat, Anxiety, Depression, and Negative Cognitive Processing did not predict PTG. Chapter Five will discuss these results as well as the limitations of the study and recommendations for future research. 


\section{CHAPTER FIVE}

\section{DISCUSSION}

This chapter includes 1) a brief summary of the study, 2) a discussion of the overall findings associated with each of the four research hypotheses and their implications, 3) limitations of the study, 4) recommendations for future research, and 5) conclusions.

\section{Summary of the Study}

Tedeschi and Calhoun (2004) defined posttraumatic growth (PTG) as "positive psychological change experienced as a result of the struggle with highly challenging life circumstances" and research investigating PTG has expanded over the past decade. More specifically, there is growing research in the field of psycho-oncology that examines PTG in the aftermath of cancer. Past research has shown promising results and has broadened the perspective on possible psychological responses following trauma. Many trauma theories (Creamer, et al., 1992; Janoff-Bulman, 1992; Park, 1998; Tedeschi \& Calhoun, 1995) propose that the degree to which trauma challenges one's beliefs and set of assumptions about the world will determine how threatening a traumatic event is, and subsequently facilitate the cognitive processing that leads to PTG. However, to the 
researcher's knowledge, few, if any, studies have directly assessed to what degree beliefs were challenged by a trauma, a concept the researcher has termed Life Outlook Threat in the present study. Rather, studies have focused on the degree of physical threat a trauma evokes and how beliefs were altered. Furthermore, while the amount of deliberate and purposeful cognitive engagement is theoretically key in the emergence of PTG, only a few studies have examined cognitive processing in relation to PTG (Gangstad, et al., 2009; Phelps, et al., 2008) Thus, there is a gap in the current literature regarding the role of threat to one's belief system and cognitive processing in the facilitation of PTG.

The overall purpose of this study was to increase understanding of how perceived threat, both cognitive and physical, as well as symptoms of anxiety and depression are associated with cognitive processing and PTG, and moreover, how cognitive processing is related to PTG in breast, prostate, and colorectal cancer survivors. Findings suggest that physical threat, along with depression, is associated with positive cognitive processing, and positive cognitive processing and life outlook threat are associated with PTG. However, Anxiety, Depression, and Negative Cognitive Processing did not emerge as predictors of PTG in the current sample of cancer survivors.

A better understanding of these factors is helpful in illuminating the mechanisms of PTG, allowing future research on the construct to be refined and furthered. In turn, more empirical support for, and a clearer understanding of the process of PTG may allow clinicians an increased ability to be aware of the potential for growth following trauma, thus helping them to better assist trauma survivors in processing their experiences and growth if and when it emerges. More broadly, research on PTG creates a more balanced view in the psychology field regarding psychological outcomes of trauma. While 
negative consequences of trauma are very real and should never be minimized or ignored, focusing exclusively on these outcomes creates a narrow view of human personality and response. With the acknowledgement and recognition of growth comes the ability to be more aware of the complexity of human personality, respectful of the multifaceted nature of trauma, and allows for a more holistic approach in treating survivors.

\section{Specific Findings and Implications for Hypotheses}

The first hypothesis in this study examined factors associated with positive cognitive processing. It stated that Anxiety and Depression would negatively predict Positive Cognitive Processing, while Perceived Threat would positively predict Positive Cognitive Processing. This hypothesis was partially supported by the data. The demographic variables accounted for $22.6 \%$ of the variance in Positive Cognitive Processing, with Interference of Treatment After-Effects emerging as a significant individual predictor in the first and second block of variables, and After-Effects of Treatment emerging as a significant predictor in all three blocks of the model. After controlling for the demographic and cancer history variables, Anxiety and Depression contributed to the model as well, accounting for an additional $18.5 \%$ of the variance in Positive Cognitive Processing. However, only Depression emerged as a significant individual predictor. Perceived Threat (composed of Life Outlook Threat and Physical Threat) accounted for an additional $5.8 \%$ of the variance in the dependent variable, yet only Physical Threat was a significant individual predictor. According to the final model, for every one point increase in the Positive Cognitive Processing mean score, Physical Threat decreased by 0.324 points, and Depression decreased by .270 points. Moreover, 
not having experienced permanent after-effects of treatment led to an increase in positive cognitive processing.

These findings contribute to the current literature, as little is known about factors associated with cognitive processing. Results suggest that lower levels of physical threat and depression, as well as reporting no permanent after-effects of cancer treatment led to an increase in positive cognitive processing. It was hypothesized that greater perceived threat would lead to positive cognitive processing, as it has been theorized (Tedeschi \& Calhoun, 2004) that a certain amount of threat is needed to provide impetus for cognitive processing. However, the results revealed the opposite. It may be that when physical threat is high a survivor is psychologically and emotionally overwhelmed and is not able to engage in the types of cognitive processing that are termed positive (i.e., Positive Cognitive Restructuring, Downward Comparison and Resolution). Furthermore, with depression often comes negative rumination and perseverative thinking (Primo, et al., 2000) and the results indicate that when depression is low the ability to engage in effortful and positive thinking increases. A similar argument can be made related to survivors experiencing permanent after-effects of treatment. It seems logical to postulate that with permanent after-effects comes greater distress and life disruption, which may obstruct a survivor's ability to think constructively. For example, significant correlations were found in the current sample between After-Effects of Treatment and Depression $(r=$ $.207, p=.008)$ as well as After-Effects of Treatment and Anxiety $(r=.206, p=.008)$. While Anxiety was not found to be a predictor of Positive Cognitive Processing in the primary model, when it was entered in the equation without Depression in a follow-up analysis, it was a significant predictor, such that for every one point increase in the 
Positive Cognitive Processing mean score, Anxiety decreased by 0.308 points. This finding suggests that lower levels of anxiety may be important in the facilitation of positive cognitive processing. Yet, the influence of Anxiety may be overtaken by the variance it shares with Depression as was the case in this sample of breast, prostate and colorectal cancer survivors.

Life Outlook Threat was not found to be a predictor of Positive Cognitive Processing. This finding was surprising as theory has suggested that growth emerges after trauma due to the event challenging one's beliefs, which leads to intentional and effortful cognitive engagement, which in turn, facilitates growth (Tedeschi \& Calhoun, 2004). Therefore, the researcher postulated that the threat to a survivor's belief system would provide the catalyst for positive cognitive processing. One possible explanation for the non-significant result is that the amount beliefs are challenged and the specific type of cognitive processing a survivor engages in are two distinct constructs. Furthermore, the degree to which one's worldview is challenged may lead to growth (see implications of Hypothesis 2) through a mechanism other than positive cognitive processing. This is a hypothesis that requires further research. In contrast, symptoms of depression and anxiety directly influenced cognition and therefore these variables were found to be related to positive cognitive processing. Moreover, a lack of physical threat may help to create the emotional stability necessary for positive types of thinking, thus explaining the inverse relationship between physical threat and positive cognitive processing. The overall results suggest that helping cancer survivors decrease their sense of perceived physical threat, as well as symptoms of depression and anxiety will increase positive cognitive processing. 
Moreover, reporting no permanent after-effects of treatment may be an indicator of an increased ability to engage in positive cognitive processing.

The second hypothesis in the present study stated that Positive Cognitive Processing and Perceived Threat would positively predict PTG, while Anxiety and Depression would negatively predict PTG. This hypothesis was partially supported by the data. The demographic control variables accounted for $16.1 \%$ of the variance in PTG, with Age and Treatment Received being significant individual predictors in the first and second block of variables. After controlling for the demographic variables as well as Anxiety and Depression, Life Outlook Threat and Physical Threat explained an additional 18.2\% of the variance in PTG, with Life Outlook Threat emerging as a significant individual predictor. When added to the model, Positive Cognitive Processing explained an additional 9.7\% of the variance in PTG. In the final model, Treatment Received, Life Outlook Threat and Positive Cognitive Processing were all significant predictors of PTG. For every one point increase in the PTG score, Life Outlook Threat increased by .452 points and Positive Cognitive Processing increased by .416 points. Also, receiving more than one form of cancer treatment was related to an increase in the PTG scores. Neither Anxiety nor Depression was related to PTG.

These findings contribute to the current body of literature, which suggests that the more beliefs are challenged by a traumatic event the more growth will emerge (Carboon, Anderson, Pollard, Szer and Seymour, 2005; Tedeschi \& Calhoun, 2004). The result that Life Outlook Threat was positively associated with PTG is important for several reasons. First, while theoretical evidence is strong, there is a lack of empirical evidence related to the degree beliefs are challenged and the emergence of growth. This finding lends 
support to theory and, in this sample, indicates that an increase in life outlook threat, or cognitive threat, is associated with increased PTG. Moreover, follow-up analyses revealed that when Life Outlook Threat and Physical Threat were entered into the regression equation together, only Life Outlook Threat was significant. However when entered separately, Physical Threat was a predictor of PTG, accounting for $2.1 \%$ of the variance in PTG. This indicates that both life outlook threat and physical threat are important in the emergence of growth, but that Life Outlook Threat may be more significant due to the fact that when it was entered into the model alone it accounted for $18.2 \%$ of the variance.

The theory of PTG, along with past research, suggests that deliberate and effortful cognitive processing is related to growth (Gangstad, Norman \& Barton, 2009; Phelps, Williams, Raichle, Turner \& Ehde, 2008; Salsman, Segerstrom, Brechting, Carlson \& Andrykowski, 2009). The results of this study lend support to this notion such that an increase in positive cognitive processing, which can be thought of as intentional and reflective thinking, was related to an increase in PTG. Thus, the degree that cancer challenged a survivor's belief system, as well as whether a survivor is engaging in positive cognitive processing may be important indicators of whether or not the individual experiences growth. Moreover, assisting in the facilitation of positive cognitive processing may facilitate growth.

It was hypothesized that a decrease in Anxiety and Depression would be related to an increase in PTG; however, neither Anxiety nor Depression were significant predictors of growth. Yet, the results from Hypothesis 1 demonstrated these variables are related to Positive Cognitive Processing. It may be the case that symptoms of anxiety and 
depression are related to positive cognitive processing, and positive cognitive processing is related to growth, but anxiety and depression are not directly related to the emergence of growth. In other words, the relationship between anxiety and growth as well as depression and growth may be explained by positive cognitive processing.

A somewhat surprising result found in Hypotheses 2 and 4 was that Treatment Received was predictive of PTG. An ANOVA conducted during the preliminary analyses revealed that receiving more than one form of cancer treatment compared to a single form of treatment led to more growth in this sample. One possible explanation for this finding is that a combination of cancer treatments creates more threat due to the potential of more side effects than any one treatment in isolation and therefore more growth emerges. An alternative explanation, and one that is supported by prior literature, is that this finding is related to gender. While Gender did not emerge as a significant individual predictor of PTG in the current sample, females reported significantly higher PTG scores compared to males $(p=.002)$, with mean scores of 53.03 and 39.18, respectively. This finding corroborates prior research (Gooden \& Winefield, 2007; Thornton \& Perez, 2006; Zwahlen, Hagenbuch, Carley, Jenewein and Buchi, 2010). To explore the potential relationship between Gender and Treatment Received, the researcher conducted a Chisquare test for independence (with Yates Continuity Correction), which indicated a significant association between the two variables, $\chi^{2}(1, \mathrm{n}=168)=11.74, p=.001$, phi $=$ -.279. The cross-tabulations of the Chi-square test indicated that the Multiple Treatment group was composed of more females (79.3\% female, $51.9 \%$ male) compared to the Single Treatment group, which was composed of more males (20.7\% female, $48.1 \%$ male). This finding suggests that survivors who have received more than one form of 
cancer treatment may report higher PTG because this group is composed of more females, rather than because of the specific treatment regimen.

An additional possibility may be that whether or not a survivor receives multiple forms of cancer treatment is associated with stage of disease, and stage of disease is in turn related to growth; e.g., prior research has suggested more advanced disease facilitates growth (Cordova, et al., 2001; Tomich \& Helgeson, 2004). Like Gender, Stage of Disease was not found to be a significant predictor of growth in the current study. Yet, a Chi-square test of independence indicated a significant association between Stage of Disease and Treatment Received, $\chi^{2}(1, \mathrm{n}=159)=13.07, p<.05$, phi $=.287$. More specifically, the cross-tabulations of the Chi-square test indicated that the Multiple Treatment group was composed of more survivors diagnosed with Stage III and IV cancer (93.3\% Stage III, 71.4\% Stage IV) compared to the Single Treatment group (6.7\% Stage III, 28.6\% Stage IV). This suggests that the relationship between receiving multiple forms of treatment and growth may be confounded by stage of disease. More research is needed to explore these possibilities.

The third hypothesis of the study stated that Anxiety, Depression, and Perceived Threat would all positively predict Negative Cognitive Processing, beyond the contribution of the demographic variables. No significant results were obtained in the regression equation; therefore this hypothesis was not supported. Based on the literature, this finding was not expected (Manne, et al., 2004; Phelps, et al., 2008; Smith, et al., 2003; Watkins, 2008). However, there are likely reasons for this outcome, as well as implications that need to be considered in future research. Most importantly, the mean score for Negative Cognitive Processing in the current sample was very low. Negative 
Cognitive Processing is calculated from the Cognitive Processing of Trauma Scale by computing a mean score from the means of the two subscales that comprise the variable, Denial and Regret. Possible scores range from zero to 12, with higher scores indicating more negative cognitive processing. The mean score in the sample was 2.74 ; thus, the survivors in the sample engaged in very little negative cognitive processing. It is worth noting that there was a fair amount of variance among scores $(M=2.74, S D=2.27)$, and this remained the case even when the five scores that fell outside two standard deviations of the mean were removed $(M=2.55, S D=2.01)$, suggesting that participants provided a fair range of responses on the variable. Nonetheless, overall the scores were low, with 42 participants (25\% of the sample) reporting Negative Cognitive Processing scores below one. In general, it is difficult to find relationships between variables when the dependent variable is not endorsed, which may explain the lack of findings. Additionally, only a few studies have used the Cognitive Processing of Trauma Scale to assess cognitive processing (Gangstad, Norman \& Barton, 2009; Phelps et al., 2008), and while the measure has been shown to have adequate reliability and validity (Williams, et al., 2002), it may not be refined to the extent necessary to detect a range of types of cognitive processing. The role of cognitive processing, both positive and negative, and how it relates to PTG, and other variables, in trauma survivors is an important field for further investigation.

The fourth hypothesis in the present study stated that Negative Cognitive Processing, Anxiety, and Depression will negatively predict PTG, while Perceived Threat will positively predict PTG. This hypothesis was partially supported by the data. The demographic variables contributed to the model and accounted for $16.5 \%$ of the variance 
in PTG, with Age and Treatment Received emerging as significant individual predictors. After controlling for the demographic variables as well as Anxiety and Depression, Life Outlook Threat and Physical Threat accounted for an additional 18.4\% of the variance, with Life Outlook Threat emerging as the significant individual predictor. In the final model, Life Outlook Threat and Treatment Received were the only significant predictors of PTG. For every one point increase in PTG score, Life Outlook Threat increased by .499 points and receiving more than one form of cancer treatment was related to an increase in the PTG score.

Anxiety, Depression and Negative Cognitive Processing did not contribute significantly to the variance in PTG. Follow-up analyses revealed that when Life Outlook Threat and Physical Threat were entered into the regression equation separately, Physical Threat was a significant individual predictor of PTG, accounting for $2.2 \%$ of the variance in PTG. As with Hypothesis 2, this result suggests that both life outlook threat and physical threat have a role in the emergence of growth, but that Life Outlook Threat may be more significant due to the fact that when it was entered into the model alone it accounted for $18.3 \%$ of the variance.

The findings from Hypothesis 4 are similar to those found with Hypothesis 2 as the same variables emerged as predictors of PTG and have the same implications (please see above). The main difference between the results of the two hypotheses is that Positive Cognitive Processing was a predictor of PTG in Hypothesis 2, while Negative Cognitive Processing was not significant in Hypothesis 4. As stated above, this result may be due to the low scoring of Negative Cognitive Processing in the sample. It is interesting to note that originally, Negative Cognitive Processing did emerge was a predictor of PTG in 
Hypothesis 4 , accounting for an additional $2.0 \%$ of the variance in PTG, $\Delta \mathrm{R}^{2}=.020, \Delta \mathrm{F}$ $(1,145)=4.68, p=.032$. Yet, upon examination of the Mahalanobis Distance statistic of the model it was determined that this result was due to an outlier in the sample and once the outlier was removed, Negative Cognitive Processing was no longer significant. Nevertheless, the initial finding suggested that a decrease in negative cognitive processing $(\beta=-.154, p=.032)$ was related to an increase in PTG. It may be the case that if the current sample of survivors engaged in higher levels of negative cognitive processing, it would have emerged as a predictor of PTG. Further research is needed to investigate this prospect.

\section{Summary of Study Implications}

The empirical literature has provided growing evidence for posttraumatic growth (PTG) following cancer. It is clear that cancer can produce negative psychological consequences; however, research has shown it can also lead to positive psychological transformation. A critical question in the PTG literature is, "what processes are most central to the emergence of growth?" The results of this study provide additional information about the relationships between several factors and PTG. The implications of the results from this study are significant to cancer survivors, psychologists and health care providers in general.

By gaining understanding of the mechanisms of growth, effective interventions can be developed and psychologists can be better equipped to help survivors not only adjust and adapt to life after trauma, but also potentially grow in ways that they otherwise may not have. Furthermore, evidence for the potential for growth following major medical illness helps to broaden the perspective on potential responses to disease and 
increases health care providers' awareness of the outcome of growth, which may lead to a more complex and nuanced approach with survivors. Calhoun and Tedeschi (1999) pointed out that experiencing growth is not the same as having an absence of negative emotions. Distress and growth often occur simultaneously, and moreover, a certain degree of distress may be needed for growth to be maintained. Thus, Calhoun and Tedeschi (1999) advised that clinicians adopt a complex perspective when working with survivors in order to enhance the possibility of growth.

When considering the implications of this study, it is important to bear in mind how PTG benefits survivors. Prior research has demonstrated that the emergence of PTG during active medical treatment for breast cancer predicted better quality of life and less distress several years after treatment was completed (Carver \& Antoni, 2004). While it may take time for growth to emerge, it might also be the case that assisting survivors in exploring benefits as they emerge early on in their cancer experience may lead to less distress over time. Yet, as stated previously, growth and distress may often co-exist, and a curvilinear relationship between the two variables has been indicated (Butler, Blasey, Garlan, McCaslin, Azarow, Chen, et al., 2005; Kleim \& Ehlers, 2009; Lechner et al., 2003; McCaslin, Zoysa, Butler, Hart, Marmar, Metzler, et al., 2009). Exploring the positive ways a survivor has changed due to his or her struggle to cope with cancer does not negate or minimize the challenges and hardships that cancer creates. Survivors who report PTG may continue to feel distress and their cancer may have a certain amount of lasting negative influence in their lives. However, simultaneously, they may feel more self-reliant, more capable, have developed deeper relationships, have a greater appreciation for life and re-prioritized their lives in a way that brings them contentment. 
The findings of this study highlight the importance of the role of Physical Threat, Depression, Anxiety and whether or not a survivor experiences permanent After-Effects of Treatment in predicting Positive Cognitive Processing. In addition, Life Outlook Threat, Positive Cognitive Processing and Type of Cancer Treatment Received were found to be associated with PTG. The results suggest that an increase in physical threat hinders positive cognitive processing, and positive cognitive processing is significant in that it predicts growth. Furthermore, an increase in life outlook threat facilitates growth. Thus, taken all together, it is important to assess a cancer survivor's perception of threat, both cognitive and physical, as well as symptoms of anxiety and depression and engagement in positive cognitive processing. It is also necessary to determine what specific types of treatment a survivor has received and whether or not he/she is experiencing permanent after-effects from treatment.

The results of the study further indicate the importance of understanding and working from a survivor's subjective point of view because key variables related to PTG, such as Life Outlook Threat, are subjective. The relationship between life outlook threat and growth indicates that from a clinical perspective, allowing survivors to explore how a cancer diagnosis challenged their general worldview may facilitate growth, as having beliefs challenged often leads to the consideration of philosophical questions regarding life. Subsequently, over time, survivors may come to question their assumptions, or believe that their beliefs pre-trauma are no longer accurate, leading them to create a set of beliefs and values that are more fitting to their new reality, which is regarded as growth. While growth should not be expected, nor should psychologists imply to survivors that those who do not report growth are somehow deficient, it may be helpful for some 
survivors to understand that while it may be initially distressing for beliefs to be challenged, or even shattered by trauma, this challenge may eventually lead to positive change and growth.

In general, the results point to the usefulness of psychosocial interventions for cancer survivors that address cognitions. Psychologists should consider opportunities to decrease symptoms of anxiety and depression as well as physical threat and the permanent after-effects of treatment as a means to manage emotional distress and increase positive cognitive processing, which in turn, would likely increase growth. The relationship between positive cognitive processing and growth suggests that constructive and deliberate cognitive engagement facilitates growth (Tedeschi \& Calhoun, 2004). Thus, it may be helpful for psychologists to assist cancer survivors in identifying ways they can engage in constructive thinking as opposed to the negative or brooding rumination that may often occur in the initial aftermath of trauma. Cognitive and relaxation based techniques may also be useful as these tend to lessen anxiety and depression, thereby increasing the ability to engage in effective cognitive processing. Perhaps most importantly, creating a safe, empathic, nonjudgmental and supportive therapeutic relationship is likely key to the facilitation of PTG. Providing survivors with the space and opportunity to freely and openly process their experiences with cancer would expectedly lead to more deliberate and constructive cognitive engagement, and subsequently, growth.

\section{Study Limitations}

While this study produced results that addressed gaps in the current psychooncology growth literature, there are limitations to the contributions. First, the results 
found cannot be generalized to all cancer, or trauma, survivors as a whole. The sample consisted of breast, prostate and colorectal cancer survivors, the majority of whom were diagnosed with Stage I or Stage II cancer. Thus, results cannot be generalized to other trauma survivors as different types of trauma may create distinct sets of circumstances. Moreover, the current sample of cancer survivors was predominately Caucasian (94.1\%), came from households with high incomes, was well educated, married, and female. (While both females and males were represented in the sample, over $69 \%$ of the sample was female) Therefore, the sample is not representative of the diversity found within the general cancer survivor population. Overall, the results of this study are limited by the lack of diversity within the sample (e.g., racial/ethnic, economic, etc.) and it would be a mistake to assume that the results can be generalized to survivors who fall outside the specific demographics of the sample.

Second, the sample appeared to be positively biased. Very low levels of negative cognitive processing, anxiety and depression were reported, while a high level of positive cognitive processing was reported. These findings might be due to socially desirable responding, or it may be the case that primarily psychologically healthy and motivated survivors were the ones who completed the survey. This seems probable if one considers that survivors who are currently experiencing high symptoms of anxiety or depression may not feel well enough or have the energy to complete the questionnaire. Overall, the sample appears to represent the most mentally healthy portion of survivors and not survivors as a general group.

Third, mailed survey research is at risk of self-selection bias and typically has a response rate of approximately $30 \%$ (Cobanoglu, Warde \& Moreo, 2001), which was the 
rate obtained in the current study. While the sample size of this study was adequate, a larger sample size would have been preferable, which can be difficult to obtain using this method. Future research should be mindful that survey research conducted through the mail might lead to positively biased and/or smaller samples and strive to sample a wider range of individuals through more efficient and/or multiple methods.

Fourth, this study used a cross-sectional research design. With this type of design, researchers are unable to draw conclusions regarding causation, and future researchers might consider a longitudinal design where survivors are assessed pre- and post-trauma, if possible, so that cause and effect can be examined. In addition, questions have been raised in the literature regarding the validity of self-reported growth (Frazier \& Kaler, 2006; Ransom, Sheldon \& Jacobsen, 2008). The measure of growth used in this study, the Posttraumatic Growth Inventory (PTGI), has established validity and reliability (Tedeschi \& Calhoun, 1996) and has been widely used in the growth literature. Nonetheless, it would be useful for future research to investigate growth using multiple methods, and multiple sources of data (e.g., data from survivors and significant others, for example).

While much effort was made to select measures that had been tested for reliability and validity within the PTG literature, there are limitations associated with two of the measures included in this study. These measures have been less widely used in research since the constructs have only recently received attention in the literature. Thus, there were few measurement options for the researcher. The Perceived Threat questionnaire was complied using several items that have been used in prior research (Cordova et al., 2001; Cordova et al., 2007; Lechner, et al., 2003; Salsman et al., 2009), although on a 
limited basis, and three items created by the researcher to assess the degree of challenge cancer poses on a survivor's worldview. These three questions were based on theory and were created based on the research's knowledge that no prior study has assessed the degree to which a trauma challenged worldview. While reliability analyses for the measure as a whole, as well as groupings of items, were conducted before the primary analyses were performed, and showed adequate reliability (please see preliminary analyses section in Chapter 4) the validity of this instrument to measure perceived threat in cancer survivors beyond the current sample has not been investigated and might be an important area for future research.

The Cognitive Processing of Trauma Scale (CPOTS), while it directly met the needs of this study, has received only a modest amount of attention in the literature. This is likely because the construct has not been empirically investigated in the growth literature to any great extent. The instrument did have adequate reliability in the current sample, and based on the alpha values it was determined that it was appropriate for the researcher to create two variables out of the scale items, Positive Cognitive Processing ( $\alpha$ $=.83)$ and Negative Cognitive Processing $(\alpha=.70)$ rather than examining each of the measure's five subscales individually. Yet, based upon prior research, the creators of the measure determined that a five factor model fit the data best (Williams et al., 2002). Thus, it is possible that the two factor model utilized in this study is not the most effective when assessing cognitive processing using the CPOTS. Furthermore, the lack of significant results related to Negative Cognitive Processing may be the result of using a measure that is potentially not adequate to identify this type of cognitive processing. At the time of this study, there were few measures assessing types of cognitive processing, 
and it is important to note limitations associated with measures that have not been widely used.

\section{Recommendations for Future Research}

This study was designed to explore the relationships between Anxiety, Depression, Perceived Threat, Cognitive Processing and PTG. Breast, prostate and colorectal cancer survivors who were at least 18 years of age, English speaking, and who did not have a history of being diagnosed with multiple types of cancer, were recruited through the Rocky Mountain Cancer Centers in the Denver metro area. It will be important for future research to focus on cancer survivors of multiple types and stages of cancer, located in a range of geographic areas, and with diverse demographic backgrounds in order to generalize findings from research.

An important area for future research is to further investigate the role of challenge to survivors' assumptive worlds as well as cognitive processing in facilitating growth as these two variables have not been widely studied in the literature. It is also imperative that future research seeks to recruit participants with a broader range of psychological health and functioning, as the current sample reported low levels of negative cognitive processing as well as depression and anxiety, thereby hindering the emergence of significant results related to these variables. It would be interesting to see if a relationship between negative cognitive processing and PTG exists in a population that reports higher levels of negative cognitive processing.

As mentioned previously, there is little information available regarding measures of perceived threat and cognitive processing as these constructs have received little empirical attention in the literature. This study contributes to this gap in research by 
creating and establishing the reliability of three items that assess how much being diagnosed with cancer challenges a survivor's assumptive world (i.e., Life Outlook Threat) and reveals a relationship between an increase in life outlook threat and an increase in PTG. Further research could consider creating additional measures of perceived threat and cognitive processing as well as more widely establishing the validity and reliability of those that exist.

Additionally, replicating findings regarding perceived threat, cognitive processing and growth would help to advance research in this important area. This study supports the finding that a decrease in physical threat and depression as well as not experiencing aftereffects of treatment predicts positive cognitive processing, and an increase in life outlook threat and positive cognitive processing predicts PTG. Receiving more than one type of cancer treatment was also found to be related to increased growth, which is interesting and somewhat unexpected. The researcher proposes that this finding may be confounded by gender and stage of disease. More research is necessary to explore these relationships and it is unknown if the overall results would be duplicated among other groups of cancer and/or trauma survivors.

Finally, using a cross-sectional research design utilizing regression analyses of the data prevents the drawing of conclusions regarding a causal relationship between the independent and dependent variables. Nonetheless, the use of multiple sources of data, both qualitative and quantitative, as well as longitudinal designs would provide researchers with a more complete understanding of the process and mechanisms of PTG and would help to establish causation. While the findings of this study are an important step in considering the predictive ability of the variables of Anxiety, Depression and 
Perceived Threat on Cognitive Processing and PTG, more complex research methods will be a necessary progression so researchers can examine multifaceted theoretical models.

\section{Conclusions}

This study examined the effect of Anxiety, Depression and Perceived Threat on Positive and Negative Cognitive Processing as well as the effect of these variables on Posttraumatic Growth (PTG) in breast, prostate and colorectal cancer survivors. Three of the four hypotheses were partially supported by the data. Results indicate that AfterEffects of Treatment, Physical Threat and Depression account for a significant amount of the variance in levels of Positive Cognitive Processing within this population, and a follow-up analysis revealed that when Anxiety was included in the regression equation without Depression, it also emerged as a significant predictor of Positive Cognitive Processing. Furthermore, Life Outlook Threat, Positive Cognitive Processing and Type of Treatment Received (single versus multiple forms of treatment) accounted for a significant amount of the variance in levels of PTG, and a follow-up analysis indicated that when Physical Threat was entered into the regression equation without Life Outlook Threat it, too, was a predictor of PTG. The hypothesis that Perceived Threat, Anxiety and Depression would positively predict Negative Cognitive Processing was not supported, which might be related to the low scoring of Negative Cognitive Processing in the current sample.

The overall findings are consistent with both the theoretical and empirical growth literature. Based upon the researcher's knowledge, Life Outlook Threat (i.e., the degree to which being diagnosed with cancer challenges a survivor's assumptive world), in particular, is a variable that has not been empirically assessed prior to this study and the 
finding that it is predictive of PTG addresses an important gap in the literature. While this study had several limitations, the results provide further evidence that receiving multiple types of cancer treatment, the emergence of permanent after-effects of treatment, perceived cognitive and physical threat, positive cognitive processing, including the role that symptoms of anxiety and depression have on positive cognitive processing, are important considerations in PTG research. 


\section{References}

Affleck, G., \& Tennen, H. (1996). Construing benefits from adversity: Adaptational significance and dispositional underpinnings. Journal of Personality, 64(4), 899-922.

Allen, J.D., Savadatti, S., \& Levy, A.G. (2009). The transition from breast cancer 'patient' to 'survivor.' Psycho-Oncology, 18, 71-78.

American Cancer Society. (2008). Cancer Facts \& Figures 2008. Retrieved April 26, 2009, from American Cancer Society, http://cancer.org/downloads/STT/2008CAFFfinalsecured.pdf

American Psychiatric Association. (2000). Diagnostic and statistical manual of mental disorders $\left(4^{\text {th }}\right.$ ed. Text Revision). Washington: Author.

Andersen, B. L., Anderson, B., \& DeProsse, C. (1989). Controlled prospective longitudinal study of women with cancer: II. Psychological outcomes. Journal of Consulting and Clinical Psychology, 57(6), 692-697.

Baker, J. M., Kelly, C., Calhoun, L. G., Cann, A., \& Tedeschi, R. G. (2008). An examination of posttraumatic growth and posttraumatic depreciation: Two exploratory studies. Journal of Loss \& Trauma, 13(5), 450-465.

Ballou, M., \& Brown, L.S. (Eds.) (2002). Rethinking mental health \& disorder: Feminist perspectives. New York: Guilford Press. 
Bellizzi, K.M. (2004). Expressions of Generativity and Posttraumatic Growth in Adult Cancer Survivors. International Journal of Aging and Human Development, 58(4), 267-287.

Bellizzi, K. M., \& Blank, T. O. (2006). Predicting posttraumatic growth in breast cancer survivors. Health Psychology, 25(1), 47-56.

Bjelland, I., Dahl, A. A., Haug, T. T., \& Neckelmann, D. (2002). The validity of the hospital anxiety and depression scale: An updated literature review. Journal of Psychosomatic Research, 52(2), 69-77.

Bonanno, G.A. (2004). Loss, trauma, and human resilience: Have we underestimated the human capacity to thrive after extremely aversive events? American Psychologist, 59, 20-28.

Bower, J., Kemeny, M., Taylor, S., \& Fahey, J. (1998). Cognitive processing, discovery of meaning, CD4 decline, and AIDS-related mortality among bereaved HIVseropositive men. Journal of Consulting and Clinical Psychology, 66(6), 979-986.

Bower, J.E., Meyerowitz, B.E., Desmond, K.A., Bernaards, C.A., Rowland, J.H. \& Ganz, P.A. (2005). Perceptions of positive meaning and vulnerability following breast cancer: Predictors and outcomes among long-term breast cancer survivors. Annals of Behavioral Medicine, 29, 236-245.

Brennan, J. (2001). Adjustment to cancer—coping or personal transition? PsychoOncology, 10(1), 1-18. 
Butler, L. D. (2007). Growing Pains: Commentary on the Field of Posttraumatic Growth and Hobfoll and Colleagues' Recent Contributions to it. Applied Psychology: An International Review, 56(3), 367-378.

Butler, L.D., Blasey, C.M., Garlan, R.W., McCaslin, S.E., Azarow, J., Chen, X., et al. (2005). Posttraumatic growth following the terrorist attacks of September 11, 2001: Cognitive, coping, and trauma symptom predictors in an internet convenient sample. Traumatology, 11, 247-267.

Calhoun, L. G., Cann, A., Tedeschi, R. G., \& McMillan, J. (2000). A correlational test of the relationship between posttraumatic growth, religion, and cognitive processing. Journal of Traumatic Stress, 13(3), 521-527.

Calhoun, L.G., \& Tedeschi, R.G. (2006). The foundations of posttraumatic growth: An expanded framework. In L.G. Calhoun, \& R.G. Tedeschi (Eds.), Handbook of posttraumatic growth: Research and practice (pp. 3-23). Mahwah, NJ: Lawrence Erlbaum Associates Publishers.

Calhoun, L.G., \& Tedeschi, R.G. (1999). Facilitating Posttraumatic Growth: A Clinician's Guide. Mahwah, NJ: Lawrence Erlbaum Associates Publishers.

Carboon, I., Anderson, V.A., Pollard, A., Szer, J., \& Seymour, J.F. (2005). Postraumatic Growth Following a Cancer Diagnosis: Do World Assumptions Contribute? Traumatology, 11, 269-283. 
Carver, C.S., \& Antoni, M.H. (2004). Finding benefit in breast cancer during the year after diagnosis predicts better adjustment 5 to 8 years after diagnosis. Health Psychology, 26, 595-598.

Cella, D. F., \& Tross, S. (1986). Psychological adjustment to survival from Hodgkin's disease. Journal of Consulting and Clinical Psychology, 54, 616-622.

Cella, D. F., \& Tross, S. (1987). Death anxiety in cancer survival: A preliminary crossvalidation study. Journal of Personality Assessment, 51(3), 451-461.

Cobanoglu, C., Warde, B., Moreo, P.J. (2001). A comparison of mail, fax and web-based survey methods. International Journal of Marketing, 405-410.

Cohen, J. (1988). Statistical power analysis for the behavioral science $\left(2^{\text {nd }}\right.$ ed.). Hillsdale, NJ: Erlbaum.

Collins, R.L., Taylor, S.E., \& Skokan, L.A. (1990). A better world or a shattered vision? Changes in life perspectives following victimization. Social cognition, 8(3), 263-285.

Cordova, M.J., Andrykowski, M.A., Kenady, D.E., et al., (1995). Frequency and correlates of posttraumatic stress disorder like symptoms and treatment for breast cancer. Journal of Consulting and Clinical Psychology, 63, 981-986.

Cordova, M. J., Cunningham, L. L. C., Carlson, C. R., \& Andrykowski, M. A. (2001). Posttraumatic growth following breast cancer: A controlled comparison study. Health Psychology, 20(3), 176-185. 
Cordova, M., Giese-Davis, Golant, Kronenwetter, Chang, \& Spiegel. (2007). Breast cancer as trauma: Posttraumatic stress and posttraumatic growth. Journal of Clinical Psychology in Medical Settings, 14(4), 308-319.

Creamer, Burgess, \& Pattison (1992). Reaction to trauma: A cognitive processing model. Journal of Abnormal Psychology, 101(3), 452-459.

Davis, C.G., Wortman, C.B., Lehman, D.R., \& Silver, R.C. (2000). Searching for Meaning in Loss: Are Clinical Assumptions Correct? Death Studies, 24, 497-540.

Dillman, D.A. (1978). Mail and telephone surveys: The total design method. New York: John Wiley \& Sons.

Dillman, D.A. (2000). Mail and internet surveys: The tailored design method $\left(2^{\text {nd }}\right.$ ed.). New York: John Wiley \& Sons.

Foa, E. B., \& Riggs, D. S. (1993). Post-traumatic stress disorder in rape victims. In J. Oldham, M. B. Riba, \& A. Tasman (Eds.), American Psychiatric Press review of psychiatry, 12, 273-303. Washington, DC: American Psychiatric Press.

Foa, E. B., \& Rothbaum, B. O. (1998). Treating the trauma of rape: Cognitive behavioral therapy for PTSD. New York: Guilford Press.

Frankl, V.E. (1959). Man's search for meaning. Boston: Beacon Press.

Frazier, P.A., \& Kaler, M.E., (2006). Assessing the validity of self-reported stress-related growth. Journal of Consulting and Clinical Psychology, 74, 859-869. 
Frazier, P., Tennen, H., Gavian, M., Park, C., Tomich, P., \& Tashiro, T. (2009). Does Self-Reported Posttraumatic Growth Reflect Genuine Positive Change? Association for Psychological Science, 20(7), 912-919.

Frazier, P.A., Tix, A.P., \& Barron, K.E. (2004). Testing moderator and mediator effects in counseling psychology research. Journal of Counseling Psychology, 51(1), 115134.

Freedman, D.A. (2005). Statistical Models. Theory and Practice. Cambridge University Press. Retrieved 04/17/2010 from http://www.stat.berkeley.edu/ census/epsilon.pdf.

Fromm, K., Andrykowski, M.A., \& Hunt, J. (1996). Positive and negative psychosocial sequelae of bone marrow transplantation: Implications for Quality of Life assessment. Journal of Behavioral Medicine, 19, 221-240.

Gangstad, B., Norman, P., \& Barton, J. (2009). Cognitive processing and posttraumatic growth after stroke. Rehabilitation Psychology, 54(1), 69-75.

Garson, D.G. (2010, March 22). Time Series Analysis StatNotes: Topics in Multivariate Analysis. Retrieved 04/06/2010 from http://faculty.chass.ncsu.edu/garson/pa765/statnote/htm.

Geffen, J. (2006). The Journey Through Cancer: Healing and Transforming the Whole Person. New York: Crown Publishers. 
Goenjian, A. K., Steinberg, A. M., Najarian, L. M., Fairbanks, L. A., Tashjian, M., \& Pynoos, R. S. (2000). Prospective study of posttraumatic stress, anxiety, and depressive reactions after earthquake and political violence. American Journal of Psychiatry, 157(6), 911-916.

Gooden, R.J. \& Winefield, H.R. (2007). Breast and Prostate Cancer Online Discussion Boards: A Thematic Analysis of Gender Differences and Similarities. Journal of Health Psychology, 12(1), 103-114.

Gray, R.E., Fitch, M., Phillips, C., Labrecque. M., \& Fergus, K. (2000). Managing the Impact of Illness: The Experiences of Men with Prostate Cancer and Their Spouses. Journal of Health Psychology, 5(4), 531-548.

Helgeson, V. S., Reynolds, K. A., \& Tomich, P. L. (2006). A meta-analytic review of benefit finding and growth. Journal of Consulting and Clinical Psychology, 74(5), 797-816.

Helgeson, V.S., Snyder, P., Seltman, H. (2004). Psychological and Physical Adjustment to Breast Cancer Over 4 Years: Identifying Distinct Trajectories of Change. Health Psychology, 23(1), 3-15.

Herr, H.W., Kornblith, A.B., Ofman, U. (1993). A comparison of the quality of life of patients with metastatic prostate cancer who received or did not receive hormonal therapy. Cancer, 71, 1143-1150. 
Herrmann, C. (1996). International Experiences with the Hospital Anxiety and Depression Scale - A Review of Validation Data and Clinical Results. Journal of Psychosomatic Research, 42(1), 17-41.

Ho, S., Chan, C., \& Ho, R. (2004). Posttraumatic Growth in Chinese Cancer Survivors. Psycho-Oncology, 13, 377-389.

Hodgkinson, P.E. \& Stewart, M. (1998). Coping with Catastrophe: A handbook of postdisaster psychosocial aftercare ( $2^{\text {nd }}$ ed.). New York: Routledge.

Hoffman, K.E., McCarthy, E.P., Recklitis, C.J., Ng, A.K. (2009). Psychological Distress in Long-Term Survivors of Adult-Onset Cancer: Results From a National Survey. Archives of Internal Medicine, 169(14), 1274-1281.

Horner MJ, Ries LAG, Krapcho M, Neyman N, Aminou R, Howlader N, Altekruse SF, Feuer EJ, Huang L, Mariotto A, Miller BA, Lewis DR, Eisner MP, Stinchcomb DG, Edwards BK (Eds). SEER Cancer Statistics Review, 1975-2006, National Cancer Institute. Bethesda, MD, http://seer.cancer.gov/csr/1975_2006/, based on November 2008 SEER data submission, posted to the SEER web site, 2009.

Horowitz, M.J., Wilner, N., \& Alvarez, W. (1979). Impact of Events Scale: A measure of subjective stress. Psychosomatic Medicine, 41, 209-218.

Horowitz, M.J. (1986). Stress response syndromes ( $2^{\text {nd }}$ ed.). Northvale, NJ: Jason Aronson. 
Janoff-Bulman, R., \& Frieze, I. H. (1983). A theoretical perspective for understanding reactions to victimization. Journal of Social Issues, 39(2), 1-17.

Janoff-Bulman, R. (1992). Shattered assumptions: Towards a new psychology of trauma. New York: Free Press.

Janoff-Bulman, R. (2004). Posttraumatic growth: Three explanatory models. Psychological Inquiry, 15(1), 30-34.

Jim, H.S., Andrykowski, M.A., Munster, P.N. \& Jacobsen, P.B. (2007). Physical Symptoms/Side Effects During Breast Cancer Treatment Predict Posttreatment Distress. Annals of Behavioral Medicine, 34(2), 200-208.

Kaiser, K. The meaning of the survivor identity for women with breast cancer. Social Science \& Medicine, 67, 79-87.

Katz, R. C., Flasher, L., Cacciapaglia, H., \& Nelson, S. (2001). The psychosocial impact of cancer and lupus: A cross validation study that extends the generality of "benefit finding' in patients with chronic disease. Journal of Behavioral Medicine, 24(6), 561571.

Kleim, B. \& Ehlers, A. (2009). Evidence for a Curvilinear Relationship Between Posttraumatic Growth and Posttrauma Depression and PTSD in Assault Survivors. Journal of Traumatic Stress, 22(1), 45-52.

Komura, K. \& Hegarty, J.R. (2006). The appraisal of positive life changes following cancer diagnosis: An interview study. Palliative and Supportive Care, 4, 3-12. 
Kornblith, A.B., Powell, M., Regan, M.M., Bennett, S., Krasner, C., Moy, B., et al. (2007). Long-term psychological adjustment in older vs younger survivors of breast and endometrial cancer. Psycho-Oncology, 16, 895-903.

Lechner, S. C., Zakowski, S. G., Antoni, M. H., Greenhawt, M., Block, K., \& Block, P. (2003). Do sociodemographic and disease-related variables influence benefit-finding in cancer patients? Psycho-Oncology, 12(5), 491-499.

Lepore, S.J. \& Helgeson, V.S. (1998). Social Constraints, Intrusive Thoughts, and Mental Health after Prostate Cancer. Journal of Social and Clinical Psychology, 17(1), 89106.

Lepore, S.J. \& Revenson, T.A. (2006). Resilience and Posttraumatic Growth: Recovery, Resistance and Reconfiguration. In L.G. Calhoun, \& R.G. Tedeschi (Eds.), Handbook of posttraumatic growth: Research and practice (pp. 24-46). Mahwah, NJ: Lawrence Erlbaum Associates Publishers.

Linley, P. A., \& Joseph, S. (2004). Positive change following trauma and adversity: A review. Journal of Traumatic Stress, 17(1), 11-21.

Lipscomb, J., Gotay, C.C., Snyder, C. (Eds.) (2005). Outcomes Assessment in Cancer: Measures, Methods, and Applications. Cambridge: Cambridge University Press.

Little, M., Paul, K., Jordens, C., Sayers, E.J. (2002). Survivorship and Discourses of Identity. Psycho-Oncology, 11, 170-178. 
Mager, W.M., \& Andrykowski, M.A. (2002). Communication in the Cancer 'Bad News' Consultation: Patient Perceptions and Psychological Adjustment. Psycho-Oncology, $11,35-46$.

Manne, S., Ostroff, J., Winkel, G., Goldstein, L., Fox, K., \& Grana, G. (2004). Posttraumatic growth after breast cancer: Patient, partner, and couple perspectives. Psychosom Med, 66(3), 442-454.

Martin, L.L., \& Tesser, A. (1996). Some Ruminative Thoughts. In R.S. Wyer (Ed.), Ruminative thought: Advances in social cognition, Vol. 9 (pp. 1-47). Mahwah, NJ: Lawrence Erlbaum Associates.

McBride, C.M., Clipp, E., Peterson, B.L., et al., (2000). Psychological impact of diagnosis and risk reduction among cancer survivors. Psycho-Oncology, 9, 418-427.

McCaslin, S.E., Zoysa, P., Butler, L.D., Hart, S., Marmar, C.R., Metzler, T.J., et al., (2009). The Relationship of Posttraumatic Growth to Peritraumatic Reactions and Posttraumatic Stress Symptoms Among Sri Lankan University Students. Journal of Traumatic Stress, 22(4), 334-339.

McMillen, J.C., Smith, E.M., \& Fisher, R.H. (1997). Perceived benefit and mental health after three types of disaster. Journal of Consulting and Clinical Psychology, 65, 733 739. 
Milam, J. (2006). Positive changes attributed to the challenge of HIV/AIDS. In L.G.

Calhoun, \& R.G. Tedeschi (Eds.), Handbook of posttraumatic growth: Research and practice (pp.138-175). Mahwah, NJ: Lawrence Erlbaum Associates Publishers.

Mineka, S. \& Sutton, S.K. (1992). Cognitive Biases and the Emotional Disorders. Psychological Science, 3(1), 65-69.

Mitchell, S. \& Campbell, E.A. (1988). Cognitions associated with anxiety and depression. Personality and Individual Differences, 9(4), 837-838.

Montazeri, A., Harirchi, I., Vahdani, M., Khaleghi, F., Jarvandi, S., Ebrahimi, M., et al. (2000). Anxiety and depression in Iranian breast cancer patients before and after diagnosis. European Journal of Cancer Care, 9(3), 151-157.

Nolen-Hoeksema, \& Davis, C. (2004). Theoretical and methodological issues in the assessment and interpretation of posttraumatic growth. Psychological Inquiry, 15(1), $60-64$.

Nunnally, J.O. (1978). Psychometric theory. New York: McGraw Hill.

Pallant, J. (2007). SPSS Survival Manual: Third Edition. New York: McGraw-Hill.

Paton, D. (2006). Posttraumatic growth in disaster and emergency work. In L.G. Calhoun, \& R.G. Tedeschi (Eds.), Handbook of posttraumatic growth: Research and practice (pp.138-175). Mahwah, NJ: Lawrence Erlbaum Associates Publishers. 
Park, C.L. (2010). Making Sense of the Meaning Literature: An Integrated Review of Meaning Making and Its Effects on Adjustment to Stressful Life Events. Psychological Bulletin, 36, 2, 257-301.

Park, C.L. (2009). Overview of Theoretical Perspectives. In Park, C.L., Lechner, S.C., Antoni, M.H., \& Stanton, A.L. (Eds.), Medical Illness and Positive Like Change: Can Crisis Lead to Personal Transformation?(pp. 11-30). Washington DC: American Psychological Association.

Park, C. L. (1998). Stress-related growth and thriving through coping: The roles of personality and cognitive processes. Journal of Social Issues, 54(2), 267-277.

Park, C.L. \& Ai, A.L. (2006). Meaning Making and Growth: New Directions for Research on Survivors of Trauma. Journal of Loss and Trauma, 11, 389-407.

Park, C.L. \& Blumberg, C.J. (2002). Disclosing trauma through writing: testing the meaning-making hypothesis. Cognitive Therapy and Research, 26(5), 597-616.

Park, C. L., \& Folkman, S. (1997). Meaning in the context of stress and coping. Review of General Psychology, 1(2), 115-144.

Park, C. L., \& Helgeson, V. S. (2006). Introduction to the special section: Growth following highly stressful life events-current status and future directions. Journal of Consulting and Clinical Psychology, 74(5), 791-796. 
Phelps, L. F., Williams, R. M., Raichle, K. A., Turner, A. P., \& Ehde, D. M. (2008). The importance of cognitive processing to adjustment in the $1^{\text {st }}$ year following amputation. Rehabilitation Psychology, 53(1), 28-38.

Primo, K., Compas, B.E., Oppedisano, G., Howell, D.C., Epping-Jordan, J.E. \& Krag, D.N. (2000). Intrusive Thoughts and Avoidance in Breast Cancer: Individual Differences and Association with Psychological Distress. Psychology and Health, 14, 1141-1153.

Ransom, S., Sheldon, K.M., \& Jacobsen, P.B. (2008). Actual Change and Inaccurate Recall Contribute to Posttraumatic Growth Following Radiotherapy. Journal of Consulting and Clinical Psychology, 76(5), 811-819.

Riskind, J.H., Williams, N.L., \& Joiner, T.E. (2006). The Looming Cognitive Style: A Cognitive Vulnerability for Anxiety Disorders. Journal of Social and Clinical Psychology, 25(7), 779-801.

Rosner, R. \& Powell, S. (2006). Posttraumatic Growth After War. In L.G. Calhoun, \& R.G. Tedeschi (Eds.), Handbook of posttraumatic growth: Research and practice (pp.138-175). Mahwah, NJ: Lawrence Erlbaum Associates Publishers.

Roy-Byrne, P., Davidson, K. W., Kessler, R. C., Asmundson, G. J. G., Goodwin, R. D., Kubzansky, L., et al. (2008). Anxiety disorders and comorbid medical illness. General Hospital Psychiatry, 30(3), 208-225. 
Rubonis, A. V., \& Bickman, L. (1991). Psychological impairment in the wake of disaster: The disaster-psychopathology relationship. Psychological Bulletin, 109(3), 384-399.

Salsman, J. M., Segerstrom, S.C., Brechting, E.M., Carlson, C.R., \& Andrykowski, M.A. (2009). Posttraumatic growth and PTSD symptomatology among colorectal cancer survivors: a 3-month longitudinal examination of cognitive processing. PsychoOncology, 18, 30-41.

Schaefer, J.A., \& Moos, R.H. (1992). Life crisis and personal growth. In B.N. Carpenter (Ed.), Personal coping: Theory, research, and application (pp. 149-170). New York: Praeger.

Schaefer, J.A., \& Moos, R.H. (1998). The context for posttraumatic growth: Life crisis, individual and social resources, and coping. In R. G. Tedeschi, C.L. Park, \& L.G. Calhoun (Eds). Posttraumatic growth: Positive changes in the aftermath of crisis (pp. 99-125). Mahwah, NJ: Lawrence Erlbaum Associates Publishers.

Sears, S. R., Stanton, A. L., \& Danoff-Burg, S. (2003). The yellow brick road and the emerald city: Benefit finding, positive reappraisal coping and posttraumatic growth in women with early-stage breast cancer. Health Psychology, 22(5), 487-497.

Seligman, M. E. P., \& Csikszentmihalyi, M. (2000). Positive Psychology: An Introduction. American Psychologist, 55(1), 5-14.

Seligman, M. E. P. (2008). Positive Health. Applied Psychology: An International Review, 57, 3-18. 
Segerstrom, S. C., Stanton, A. L., Alden, L. E., \& Shortridge, B. E. (2003). A multidimensional structure for repetitive thought: What's on your mind, and how, and how much? Journal of Personality and Social Psychology, 85(5), 909-921.

Sinding, C. \& Gray, R. (2005). Active aging-spunky survivorship? Discourses and experiences of the years beyond breast cancer. Journal of Aging Studies, 19, 147-161.

Smith, E. M., Gomm, S. A., \& Dickens, C. M. (2003). Assessing the independent contribution to quality of life from anxiety and depression in patients with advanced cancer. Palliative Medicine, 17(6), 509-513.

Somerfield, M. R., Stefanek, M. E., Smith, T. J., \& Padberg, J. J. (1999). A systems model for adaptation to somatic distress among cancer survivors. Psycho-Oncology, 8(4), 334-343.

Stanton, A.L., Bower, J.E., \& Low, C.A. (2006). Posttraumatic growth after cancer. In L.G. Calhoun, \& R.G. Tedeschi (Eds.), Handbook of posttraumatic growth: Research and practice (pp.138-175). Mahwah, NJ: Lawrence Erlbaum Associates Publishers.

Sumalla, E.C., Ochoa, C., \& Blanco, I. (2009). Posttraumatic growth in cancer: Reality or illusion? Clinical Psychology Review, 29, 24-33.

Surveillance, Epidemiology, and End Results Program, 1975-2004, Division of Cancer Control and Population Sciences, National Cancer Institute, 2007.

Tabachnick, B.G., \& Fidell, L.S. (2007). Using Multivariate Statistics: Fifth Edition. Boston: Pearson Education, Inc. 
Tallman, B. A., Altmaier, E., \& Garcia, C. (2007). Finding benefit from cancer. Journal of Counseling Psychology, 54(4), 481-487.

Taylor, S. E. (1983). Adjustment to threatening events: A theory of cognitive adaptation. American Psychologist, 38(11), 1161-1173.

Taylor, S.E. \& Brown, J.D. (1988). Illusion and well-being: A social psychological perspective on mental health. Psychological Bulletin, 103, 193-210.

Tedeschi, R.G., \& Calhoun, L.G. (1995). Trauma and transformation: Growing in the aftermath of suffering. Thousand Oaks, CA: Sage.

Tedeschi, R.G., \& Calhoun, L.G. (1996). The Posttraumatic Growth Inventory: Measuring the positive legacy of trauma. Journal of Traumatic Stress, 9, 455-471.

Tedeschi, R.G., Park, C.L., \& Calhoun, L.G. (Eds.) (1998). Posttraumatic growth: Positive changes in the aftermath of crisis. Mahwah, NJ: Lawrence Erlbaum Associates.

Tedeschi, R. G., \& Calhoun, L. G. (2004). Target article: 'posttraumatic growth: Conceptual foundations and empirical evidence'. Psychological Inquiry, 15(1), 1-18.

Tedeschi, R.G., \& Kilmer, R. P. (2005). Assessing Strengths, Resilience, and Growth to Guide Clinical Interventions. Professional Psychology: Research and Practice, 36(3), $230-237$. 
Thomas, S.F., Glynne-Jones, R., Chait, I., \& Marks, D.F. (1997). Anxiety in Long-Term Cancer Survivors Influences the Acceptability of Planned Discharge from Follow-Up. Psycho-Oncology, 6, 190-196.

Thornton, A. A., \& Perez, M. A. (2006). Posttraumatic growth in prostate cancer survivors and their partners. Psycho-Oncology, 15(4), 285-296.

Tomich, P. L., \& Helgeson, V. S. (2004). Is finding something good in the bad always good? Benefit finding among women with breast cancer. Health Psychology, 23(1), $16-23$.

Ullrich, P. M., \& Lutgendorf, S. K. (2002). Journaling about stressful events: Effects of cognitive processing and emotional expression. Annals of Behavioral Medicine, 24(3), 244-250.

Vickberg, S.M.J., Bovbjerg, D.H., DuHamel, K.N., Currie, V., \& Redd, W.H. (2000). Intrusive Thoughts and Psychological Distress Among Breast Cancer Survivors: Global Meaning as a Possible Protective Factor. Behavioral Medicine, 25, 152-160.

Watkins, E. R. (2008). Constructive and unconstructive repetitive thought. Psychological Bulletin, 134(2), 163-206.

Watson, D., Clark, L.A., Weber, K., Assenheimer, J.S., Strauss, M.E. \& McCormick, R.A. (1995). Testing a tripartite model: II. Exploring the symptom structure of anxiety and depression in student, adult and patient samples. Journal of Abnormal Psychology, 104, 15-25. 
Weisman, A.D., \& Worden, J.W. (1976). The existential plight in cancer: significance of the first 100 days. International Journal Psychiatry in Medicine, 7(1), 1-15.

Widows, M. R., Jacobsen, P. B., Booth-Jones, M., \& Fields, K. K. (2005). Predictors of posttraumatic growth following bone marrow transplantation for cancer. Health Psychology, 24(3), 266-273.

Williams-Avery, Rhonda Maetel (1999). Cognitive mechanisms linking disclosure, inhibition and self-reported health. Ph.D. dissertation, Arizona State University, United States - Arizona. Retrieved April 26, 2009, from Dissertations \& Theses: Full Text database. (Publication No. AAT 9924214).

Williams, R.M., Davis, M.C., \& Millsap, R.E. (2002). Development of the Cognitive Processing of Trauma Scale. Clinical Psychology and Psychotherapy, 9, 349-360.

Wortman, C.B. (2004). Posttraumatic Growth: Progress and Problems. Psychological Inquiry, 15(1), 81-90.

Zabora, J., Brintzenhofeszoc, K., Curbow, B., Hooker, C., \& Piantadosi, S. (2001). The prevalence of psychological distress by cancer site. Psycho-Oncology, 10(1), 19-28.

Zigmond, A.S. \& Snaith, R. P. (1983). The hospital anxiety and depression scale. Acta Psychiatr Scand, 67, 361-370.

Znoj, H. (2006). Bereavement and Posttraumatic Growth. In L.G. Calhoun, \& R.G. Tedeschi (Eds.), Handbook of posttraumatic growth: Research and practice (pp.138175). Mahwah, NJ: Lawrence Erlbaum Associates Publishers. 
Zoellner, T., \& Maercker, A. (2006). Posttraumatic growth in clinical psychology-A critical review and introduction of a two component model. Clinical Psychology Review, 26(5), 626-653.

Zwahlen, D., Hagenbuch, N., Carley, M.I., Jenewein, J., \& Buchi, S. (2010).

Posttraumatic growth in cancer patients and partners-effects of role, gender and the dyad on couples' posttraumatic growth experience. Psycho-Oncology, 19, 12-20. 


\section{Appendix A}

\section{Glossary of Terms}

Anxiety. In the study, anxiety is defined as only the cognitive symptoms of anxiety, to prevent overlap with physical symptoms that are often related to illness, such as dizziness, and fatigue (Bjelland, Dahl, Haug, \& Neckelmann, 2002).

Assumptive world. Assumptive world is defined as the general set of beliefs, and assumptions about the world, individuals develop and rely on to guide their actions, help them to understand the causes and reasons for events, and that can provide them with a general sense of meaning and purpose (Tedeschi \& Calhoun, 2004).

Avoidance. Avoidance is defined as "cognitive or behavioral efforts aimed at preventing thoughts and memories from entering conscious awareness when they become too overwhelming. Avoidance can involve both intentional efforts (e.g., trying not to think about an unwanted thought) as well as seemingly unintended responses (e.g., a sense that one's emotions are numb) (Primo, et al., 2000, p. 1142). Both avoidance and intrusive thoughts (see below) are regarded as problematic when they are prolonged or excessive, suggesting an inability to integrate a traumatic experience with pre-existing cognitive schemas (as cited in Primo, et al., 2000).

Cognitive processing. Cognitive processing is defined as "the process of actively thinking about a stressor, the thoughts and feelings it evokes, and its implications for one's life and future" (Bower, Kemeny, Taylor, \& Fahey, 1998, p.979). 
Deliberate rumination. Deliberate rumination is defined as effortful and purposeful thinking that might include reminiscing, problem solving, and trying to make sense out of a situation. This process tends to repair or restructure the individual's general way of understanding the world (Tedeschi \& Calhoun, 2006).

Depression. Similar to anxiety, in the study depression is defined as only the cognitive symptoms of depression, and is primarily related to the anhedonic state that often accompanies depression in order to distinguish between symptoms related to depression versus those related to cancer (Bjelland, Dahl, Haug, \& Neckelmann, 2002; Zigmond \& Snaith, 1983).

Intrusive thoughts. Intrusive thoughts include involuntary thoughts, images and dreams that can be associated with positive or, more frequently, negative emotions in response to a stressor. They are triggered by internal or external stimuli related to the original stressor and experienced as beyond personal control (Primo, et al., 2000).

Life Outlook Threat. In the study, Life Outlook Threat, in relation to the theory of PTG, is defined as the degree to which the traumatic event is challenging to an individual's assumptive world (Tedeschi \& Calhoun, 2004).

Mortality salience. In the study, mortality salience is defined as the perceived degree being diagnosed with cancer threatens an individual's life, and the degree to which he/she reacted with feelings of intense fear, horror, or helplessness (DSM-IV-TR; American Psychiatric Association, 2000).

Negative cognitive Processing. Negative cognitive processing is defined by two of the subscales within the Cognitive Processing of Trauma Scale; Denial and Regret (Williams et al., 2002). 
Perceived Threat. In the study, perceived threat is defined as the degree to which being diagnosed with cancer threatens a survivor's sense of physical well-being. Perceived threat consists of two main components: mortality salience and reacting with feelings of intense fear and helplessness.

Positive cognitive processing. Positive cognitive processing is defined by three of the subscales within the Cognitive Processing of Trauma Scale; Positive cognitive restructuring, Resolution, and Downward comparison (Williams et al., 2002).

Posttraumatic growth. Posttraumatic growth is defined as "the individual's experience of significant positive change resulting from the struggle with a major life crisis" (Calhoun, Cann, Tedeschi, \& McMillian (2000), p. 521). Tedeschi and Calhoun (1996) divided posttraumatic growth into five domains: personal strength, new possibilities, relating to others, appreciation of life, and spiritual change.

Rumination. Rumination is generally defined as "a class of conscious thoughts that revolve around a common instrumental theme and that recur in the absence of immediate environmental demands requiring the thoughts" (Martin \& Tesser, 1996, p.1).

Survivor. In the study, survivor is defined as a person who remains alive to function during and after overcoming a serious hardship or life-threatening disease. In cancer, a person is considered to be a survivor from the time of diagnosis until the end of life (National Cancer Institute, 2009).

Trauma/Traumatic Event. In the study, trauma is defined as a set of circumstances that represent significant challenges to the adaptive resources of the individual, as well as to the individual's way of understanding the world and his/her place in it (Janoff-Bulman, 1992). 
Appendix B

Informed Consent

\title{
Posttraumatic Growth Following Cancer: The Role of Cognitive Processing, Anxiety, Depression and Perceived Threat
}

\author{
Principal Investigator: Jennifer Caspari, MA \\ Co-Investigators: Teri Simoneau, $\mathrm{PhD}$ \\ Susan Ash-Lee, MSW, LCSW
}

\section{INTRODUCTION}

You are invited to take part in a research study that is a doctoral dissertation conducted by Jennifer M. Caspari, MA, a counseling psychology doctoral student at University of Denver. This study is being conducted in partnership with the Rocky Mountain Cancer Centers (RMCC). In addition, it is being supervised by Dr. Cynthia McRae, PhD, Professor of Counseling Psychology, University of Denver, Denver, CO, 80208, 303871-2475, cmcrae@du.edu.

You are being asked to participate because you are a cancer survivor. Your participation in this study is entirely VOLUNTARY. You should read the information below before deciding whether or not to participate.

\section{PURPOSE OF STUDY}

The purpose of this study is to explore factors that contribute to positive changes following cancer. It is important to learn more about factors that lead to positive change and growth after cancer. Such information can be used to develop useful interventions that recognize the complexity of the cancer experience, and better help people with cancer.

\section{PROCEDURES}

If you volunteer to participate in this study, we will ask you to do the following things:

- Read and sign this form. Your consent to participate in the study is given when you sign and return this form. A second identical form is enclosed for you to keep for your reference.

- Read and sign the enclosed HIPAA Research Authorization Form. A second identical HIPAA form is enclosed for you to keep for your reference. 
- Fill out the enclosed questionnaires about your demographic information, mood, thoughts and experiences related to cancer, and changes you may have experienced following cancer. The questionnaire will take approximately 20 to 30 minutes to complete. You will fill out this questionnaire just once.

- Return the signed consent form, signed HIPAA form and the completed questionnaire in the pre-addressed, postage-paid envelope provided.

\section{POTENTIAL RISKS AND DISCOMFORTS}

The risks associated with this project are minimal. However, sometimes people experience mild emotional distress or embarrassment when asked to think about their thoughts and feelings related to their cancer experience. Filling out the questionnaires may evoke unpleasant feelings related to your cancer experience, or you may feel burdened by filling out the questionnaires. You are encouraged to participate only if you feel that filling out these questionnaires will not be a burden. While we encourage you to answer every question, we respect your right to choose not to answer any questions that make you feel uncomfortable. If you become upset by participating in the study, you may contact the co-investigators: Teri Simoneau, PhD (303) 285-5082 or Susan Ash-Lee, MSW, LCSW (303) 285-5076 to be given a referral for psychological counseling. You will be responsible for the cost of counseling.

\section{ANTICIPATED BENEFITS TO SUBJECTS}

Because individuals respond differently, no one can know in advance if participation will be helpful in your particular case. The potential benefits may include gaining more understanding regarding your cancer experience.

\section{ANTICIPATED BENEFITS TO SOCIETY}

The possible benefits from this study to society could include developing better interventions that provide support to cancer patients and survivors.

\section{ALTERNATIVES TO PARTICIPATION}

You may discontinue the study at any time and still have the right to other treatments available to you. The questionnaires given as part of this study are unrelated to any care you may be receiving and are solely part of this research study.

\section{PAYMENT FOR PARTICIPATION}

You will receive no money for participation in this study. 


\section{FINANCIAL OBLIGATION}

Neither you nor your insurance company will be billed for you participation in this research.

\section{PRIVACY AND CONFIDENTIALITY}

We will make every effort to keep your research records confidential. You will be assigned an "identification number" and this will be used for all questionnaires and data analysis. The list that identifies your name with your identification number will be kept in a locked file separate from your questionnaire data. Please do NOT include your name anywhere on the questionnaire.

Records that identify you may be looked at by the following people:

- Federal agencies that oversee human subject research

- University of Denver Institutional Review Board

- HCA-HealthONE Institutional Review Board

- The investigators and research team for this study

- Regulatory officials from the institution where the research is being conducted, to ensure compliance with policies or monitor the safety of the study.

The results of this research may be presented at meetings or in published articles; however, your name will be kept private. Information collected during the research study will be kept in a secure computer system. After your participation in the study is complete, you will be identified only by code number. Any protected health information will be kept under lock and key.

\section{PARTICIPATION AND WITHDRAWAL}

Your participation in this research is VOLUNTARY. Consent to participate in this research, and the use of the answers you supply, is given when you return your signed consent form and completed questionnaire by mail.

If you choose not to participate, you may simply throw away this questionnaire. Your relationship with Rocky Mountain Cancer Centers, or your right to health care or other services to which you are otherwise entitled, will not be affected. You can discontinue participation at any time without affecting your future care.

- If you choose not to participate, please take a moment and return the preaddressed, postage-paid refusal card. 


\section{IDENTIFICATION OF INVESTIGATORS}

If you have any questions or concerns about this research, or if you experience a negative reaction to this study, please feel free to contact the principal investigator:

Principal Investigator: Jennifer Caspari, MA (720) 468-118, jennifer.caspari@gmail.com

If you are in need of another questionnaire you may contact the co-investigators:

Co-Investigators: Teri Simoneau, PhD (303) 285-5082 and Susan Ash-Lee, MSW, LCSW (303) 285-5076

\section{RIGHTS OF RESEARCH SUBJECTS}

You may discontinue participation and simply throw away this questionnaire at any time without penalty. You are not waiving any legal claims, rights or remedies because of your participation in this research study. If you have questions regarding your rights as a research subject, you may contact Susan Sadler, Chair, University of Denver Institutional Review Board for the Protection of Human Subjects, at 303-871-3454, or Sylk SottoSantiago, Office of Research and Sponsored Programs at 303-871-4052 or write to either at the University of Denver, Office of Research and Sponsored Programs, 2199 S.

University Blvd., Denver, CO 80208-2121. Or you may contact the Vice Chairman of the HCA-HealthONE Institutional Review Board, Carol Greenwald, M.D. at 303-584-2300.

Please sign below if you understand and agree to the above. If you do not understand any part of the above form, please ask the prinicipal investigator (Jennifer Caspari, MA (720) 468-1118, jennifer.caspari@gmail.com) any questions you have.

Please return this signed form, signed HIPAA form and your completed questionnaire in the return envelope provided. Keep the enclosed identicial copy of this form and the HIPAA form for your reference.

I have read and understood the abovementioned descriptions of the study called Posttraumatic Growth Following Cancer: The Role of Cognitive Processing, Anxiety, Depression and Perceived Threat. I have asked for and received a satisfactory explanation of any language that I did not fully understand. I agree to participate in this study, and I understand that I may withdraw my consent at any time. I have received a copy of this consent form.

Printed Name of Participant

Signature of Participant

Date 


\section{Investigator's Statement:}

I have made myself available to answer and explain the research to the patient or legally authorized representative and answered all questions. I believe that he-she understand the information described in this informed consent and freely consents to participation.

Printed Name of Investigator

Date 


\section{Appendix C}

\section{HIPAA Research Authorization}

\section{Authorization to use and Disclose Health Information}

This section explains who will use and share your study-related health information is you agree to be in this study.

By signing this Authorization, you agree to allow the use and sharing of you health information as described below.

1. During the study, the study doctor and study staff will use, collect, and share health information about you. The health information that may be used and shared includes:

- All information collected during the research study and procedures described in the Informed Consent Form; and

- Personal health information in your medical records that is relevant to the study, which includes your past medical history, medical information from your primary care physician, and other medical information relating to your participation in the study.

2. The study doctor and study staff may share your health information with:

- Representatives of the government agencies in the United States and other countries (including the FDA)

- Review boards and other persons who watch over the safety, effectiveness, and conduct of the study and

- The sponsor of the study and its affiliates, agents, and contractors assisting in the conduct of completion of the study.

These people will use your records to review the study, check the safety and results of the study, and check how researchers are doing the study.

3. The study doctor, study staff, or sponsor may use some facts about your being in this study in books, magazines, journals, and scientific meetings. If this happens, no one will use your name or other information that could be used to identify you.

4. The study doctor and study staff may share your health information with your health care payer to resolve your claim if you are hurt because of being in this study. If this happens, the study doctor or the sponsor may share your health information with their insurance carriers to resolve your insurance claim, and the study doctor may also request medical records from your other health care providers to learn more about your condition. 
5. The study doctor and study staff will share your health information with your health care payer in order to collect payment for costs (even if your health care payer does not cover these costs).

6. Once your health information has been disclosed to a third party, federal privacy laws may no longer protect it from further disclosure. After the study doctor or study staff shares your health information with the sponsor or others, the sponsor or others may share your records with other people who do not have to protect the privacy of your health information.

If you would like to know how the sponsor will protect the privacy of your health information, ask the study doctor how to get this information.

Please note that the study doctor or study staff may share personal information about you if required by law. (For example, if the study doctor or study staff suspects that you are going to harm someone or yourself.) If you have questions about this, please ask the study doctor.

You do not have to sign this Authorization, but if you do not, you may not participate in the study. If you do not sign this Authorization, your right to medical treatment will not be affected.

You may change your mind and revoke (take back) this Authorization at any time for any reason. To revoke this Authorization, you must write a letter to the study doctor.

However, if you revoke this Authorization, you will not be allowed to continue taking part in the study. Also, even if you revoke this Authorization, the study doctor and study staff may continue to use and share the health information they have already collected to protect the integrity of the study.

While the study is in process, you may not be allowed to see your health information that is created or collected by the study doctor and study staff during the course of the study. After the study is finished, however, you may see and copy this information.

This Authorization does not have an expiration (ending) date. 
You will be given a copy of this Authorization after you have signed it.

Indicate your agreement to the use and sharing of your study-related health information by signing below:

Printed Name of Participant

Date

Signature of Participant

Time 


\section{Appendix D}

\section{Demographics}

Please answer the following questions about yourself. These will be used for statistical analysis only.

For the following questions, please place a checkmark in the area to the left of the option that best applies to you.

\section{Part 1: Background Information}

1. What is your date of birth?

2. What is your gender?

Female

Male

Transgender

3. Which of the following categories below do you feel best describes your race or ethnicity?

Caucasian

Hispanic, Latino/a

African-American

Asian or Pacific Islander

American Indian or Alaskan Native

Other (please indicate)

4. What is your marital status? (Specify only one)

Single (never married)

Committed relationship

Married/Remarried

Divorced/Separated/Widowed

Other (please specify) 
5. What is your current living arrangement? (Specify only one)

Live alone

Live with spouse or partner, with children

Live with spouse or partner, without children

Live with children

Live with someone else (please specify)

Other (please specify)

6. How many years of school have you completed? (please circle the number that best explains your level of education)

$\begin{array}{llllllllllll}8 & 9 & 10 & 11 & 12 & 13 & 14 & 15 & 16 & 17 & 18 & 19+\end{array}$

Other (please specify)

7. Approximately, what is your household income?

Check one income range that best describes your household income for last year from all sources of income (salaries, wages, tips, social security, disability income or insurance, retirement income, or any other income).

Under $\$ 25,000$

$\$ 25,001-\$ 50,000$

$\$ 50,001-\$ 75,000$

$\$ 75,001-\$ 100,000$

$\$ 100,000+$

\section{Part 2: Questions about Cancer Diagnosis and Treatment}

8. When were you first diagnosed with cancer?

MONTH YEAR

9. What type of cancer were you diagnosed with?

Breast

Prostate

Colorectal

Other 
10. What stage was your cancer at initial diagnosis?

Stage I

Stage II

Stage III

Stage IV

Other (please explain)

11. Where did you receive your cancer treatment? (Or where are you receiving treatment if on maintenance medication). Please specify specific location. (Example: Rocky Mountain Cancer Centers Denver-Midtown)

12. What treatment did you receive for your cancer? (check all that apply)

Radiation

Chemotherapy

Surgery (please specify)

Other (please explain)

13. How long has it been since you finished your primary treatment? (Primary treatment refers to radiation, chemotherapy, or surgery, not maintenance medication)

2 - 4 months

5 - 6 months

7 - 12 months

13 - 36 months

Other (please specify)

14. What medications are you CURRENTLY taking? (check all that apply)

Arimidex

Tamoxifen

Aromasin

Femara

Zometa

Faslodex

Tykerb

Lupron

15. a. Have you experienced a recurrence of cancer?

yes

no 
b. If you checked YES, when did you experience a recurrence?

MONTH__ YEAR

c. If you checked YES, how many recurrences have you had?

1- 2

more than 2

Other

16. a. Right now do you have any permanent after effects from treatment?

yes

no

b. If you checked YES, what are they?

c. If you checked YES, how much do the after effects interfere with your daily activities?

none a little

some

a lot 


\section{Appendix E}

\section{Perceived Threat}

Some people with cancer think that being diagnosed with cancer, as well as the general cancer experience, represents a threat to their physical well-being, mortality, and the way they see the world. The following set of questions asks you about the threat of your cancer experience.

For each question, please circle the number from 1 to 6 that best describes your experiences.

\section{Life Outlook:}

Some people think cancer challenges the way they see themselves, others, and the world. The following questions address this issue.

1. To what extent did being diagnosed with cancer challenge the way you see your self
1
2
3
4
5
6

not at all

extremely

2. To what extent did being diagnosed with cancer challenge the way you see others?
1
2
3
4
5
6

not at all

extremely

3. To what extent did being diagnosed with cancer challenge the way you see the world in general?
1
2
3
4
5
6

not at all

extremely 


\section{Physical Threat:}

Some people with cancer think about cancer's effect on their mortality. The following questions address this issue.

4. Please try to recall how you felt when you were first diagnosed with cancer. At that time, how likely did you think it was that you would die of cancer?
1
2
3
4
5
6

not at all

extremely

5. How likely do you think it is that you will develop cancer again, or that your cancer will progress in your lifetime?
1
2
3
4
5
6

not at all

extremely

6. How likely do you think it is that you will die of cancer?
1
2
3
4
5
6

not at all

extremely

\section{Reaction to Cancer:}

Some people with cancer react to the experience with feelings of potential threat and fear. The following questions address this issue.

7. In response to your cancer experience, have you felt that the event was a potential threat to your life and safety?
1
2
3
4
5
6

not at all

extremely

8. In response to your cancer experience have you reacted with feelings of intense fear, helplessness, or horror?
1
2
3
4
5
6

not at all

extremely 


\section{Appendix F}

\section{Hospital Anxiety and Depression Scale}

The following set of questions asks you about how you feel. Read each item below and check the reply which comes closest to how you have been feeling in the past week.

Don't take too long over your replies; your immediate reaction to each item will probably be more accurate than a long, thought-out response.

1. I feel tense or 'wound up'

Most of the time

A lot of the time

From time to time, occasionally

Not at all

2. I still enjoy things I used to enjoy

Definitely as much

Not quite so much

Only a little

Hardly at all

3. I get a sort of frightened feeling as if something awful is about to happen Very definitely and quite badly Yes, but not too badly A little, but it doesn't worry me Not at all

4. I can laugh and see the funny side of things

As much as I always could Not quite so much now Definitely not so much now Not at all

5. Worrying thoughts go through my mind A great deal of the time A lot of the time Not too often Very little

6. I feel cheerful

Never

Not often

Sometimes

Most of the time 
7. I can sit at ease and feel relaxed

Definitely

Usually

Not often

Not at all

8. I feel as if I am slowed down

Nearly all the time

Very often

Sometimes

Not at all

9. I get a sort of frightened feeling like 'butterflies' in the stomach

Not at all

Occasionally

Quite often

Very often

10. I have lost interest in my appearance

Definitely

I don't take as much care as I should

I may not take quite as much care

I take just as much care as ever

11. I feel restless as if I have to be on the move

Very much indeed

Quite a lot

Not very much

Not at all

12. I look forward with enjoyment to things

As much as I ever did

Rather less than I used to

Definitely less than I used to

Hardly at all

13. I get sudden feelings of panic

Very often indeed

Quite often

Not very often

Not at all 
14. I can enjoy a good book or radio or television program Often Sometimes

Not often

Very seldom 


\section{Appendix G}

\section{Cognitive Processing of Trauma Scale}

The following set of questions asks you about your experience with cancer. Please rate the extent to which you agree with each of the following statements, using the following rating sale.

-3 , strongly disagree

-2 , moderately disagree

-1 , slightly disagree

0 , neither mainly agree nor disagree

1 , slightly agree

2, moderately agree

3 , strongly agree

1. There is ultimately more good than bad in this experience

2. I have figured out how to cope

3. I say to myself 'this isn't real'

4. I have moved on and left this event in the past

5. Overall, this event feels resolved for me

6. I have comes to terms with this experience

7. I often think, 'if only I had done something different'

8. I blame myself for what happened

9. I refuse to believe that this really happened to me

10. I wish I could have handled this differently

11. Other people have had worse experiences than mine

12. I act as if this event never really happened

13. Even though my experience was difficult, I can think of ways that it could have been worse

14. My situation is not so bad compared to other peoples' situations

15. I am able to find positive aspects of this experience 
16. I have been able to find a 'silver lining' in this event

17. I pretend this didn't really happen 


\section{Appendix $\mathrm{H}$}

\section{The Posttraumatic Growth Inventory}

People sometimes find that a crisis such as cancer may eventually lead to positive changes in their lives. For each of the items below, indicate the degree to which the changes described in the items has occurred in your life-as of today-as a result of cancer, using the following scale:

$0=\mathrm{I}$ did not experience this change as a result of my cancer.

$1=\mathrm{I}$ experienced this change to a very small degree as a result of my cancer.

$2=\mathrm{I}$ experienced this change to a small degree as a result of my cancer.

$3=\mathrm{I}$ experienced this change to a moderate degree as a result of my cancer.

$4=\mathrm{I}$ experienced this change to a great degree as a result of my cancer.

$5=\mathrm{I}$ experienced this change to a very great degree as a result of my cancer.

1. I changed my priorities about what is important in life.

2. I have a greater appreciation for the value of my own life.

3. I developed new interests.

4. I have a greater feeling of self-reliance.

5. I have a better understanding of spiritual matters.

6. I more clearly see that I can count on people in times of trouble.

7. I established a new path for my life.

8. I have a greater sense of closeness with others.

9. I am more willing to express my emotions.

10. I know better that I can handle difficulties.

11. I am able to do better things with my life.

12. I am better able to accept the way things work out.

13. I can better appreciate each day.

14. New opportunities are available which wouldn't have been otherwise.

15. I have more compassion for others. 
16. I put more effort into my relationships.

17. I am more likely to try to change things which need changing.

18. I have a stronger religious faith.

19. I discovered that I'm stronger than I thought I was.

20. I have learned a great deal about how wonderful people are.

21. I better accept needing others. 